\title{
On Landmark Densities in Minimum-Uncertainty Motion Planning
}

Jonas Nordlöf 



\section{On Landmark Densities in Minimum-Uncertainty Motion Planning}

Jonas Nordlöf

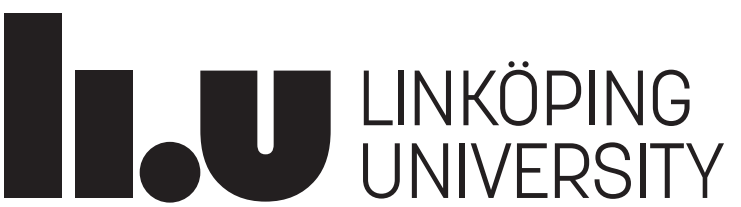


This is a Swedish Licentiate's Thesis.

Swedish postgraduate education leads to a Doctor's degree and/or a Licentiate's degree.

A Doctor's Degree comprises 240 ECTS credits (4 years of full-time studies).

A Licentiate's degree comprises 120 ECTS credits, of which at least 60 ECTS credits constitute a Licentiate's thesis.

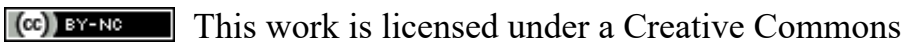
Attribution-NonCommercial 4.0 International License. https://creativecommons.org/licenses/by-nc/4.0/

Linköping studies in science and technology. Licentiate Thesis

No. 1927

On Landmark Densities in Minimum-Uncertainty Motion Planning

Jonas Nordlöf

\author{
jonas.nordlofeliu.se \\ www. control.isy.liu.se \\ Department of Electrical Engineering \\ Linköping University \\ SE-581 83 Linköping \\ Sweden
}

ISBN 978-91-7929-244-7 (Print)

ISBN 978-91-7929-245-4 (PDF)ＩSSN 0280-7971

Copyright (C) 2022 Jonas Nordlöf

Printed by LiU-Tryck, Linköping, Sweden 2022 
To our future robot overlords! 



\section{Abstract}

Accurate self-positioning of autonomous mobile platforms is important when performing tasks such as target tracking, reconnaissance and resupply missions. Without access to an existing positioning infrastructure, such as Global Navigation Satellite Systems (GNSS), the platform instead needs to rely on its own sensors to obtain an accurate position estimate. This can be achieved by detecting and tracking landmarks in the environment using techniques such as simultaneous localization and mapping (SLAM). However, landmark-based SLAM approaches do not perform well in areas without landmarks or when the landmarks do not provide enough information about the environment. It is therefore desirable to estimate and minimize the position uncertainty while planning how to perform the task. A complicating factor is that the landmarks used in SLAM are not known at the time of planning.

In this thesis, it is shown that by integrating SLAM and path planning, paths can be computed that are favorable, from a localization point of view, during motion execution. In particular, it is investigated how prior knowledge of landmark distributions, or densities, can be used to predict the information gained from a region. This is done without explicit knowledge of landmark positions. This prediction is then integrated into the path-planning problem.

The first contribution is the introduction of virtual landmarks which represent the expected information in unexplored regions during planning. Two approaches to construct the virtual landmarks that capture the expected information available, based on the beforehand known landmark density, are given. The first approach can be used with any sensor configuration while the second one uses properties of range-bearing sensors, such as LiDAR sensors, to improve the quality of the approximation.

The second contribution is a methodology for generating landmark densities from prior data for a forest scenario. These densities were generated from publicly available aerial data used in the Swedish forest industry.

The third contribution is an approach to compute the probability of detecting pole-based landmarks in LiDAR point clouds. The approach uses properties of the sensor, the landmark detector, and the probability of occlusion from other landmarks in order to model the detection probability. The model accuracy has been validated in simulations where a real landmark detector and simulated LiDAR point clouds have been used in a forest scenario.

The final contribution is a position-uncertainty aware path-planning approach. This approach utilizes virtual landmarks, the landmark densities, and the landmark detection probabilities, to produce paths which are advantageous from a positioning point of view. The approach is shown to reduce the platform position uncertainty in several different simulated scenarios without prior knowledge of explicit landmark positions. The computed position uncertainty is shown to be relatively comparable to the uncertainty obtained when executing the path. Furthermore, the generated paths show characteristics that make sense from an application point of view. 



\section{Populärvetenskaplig sammanfattning}

Positionering av autonoma mobila plattformar är viktigt för att utföra uppgifter som utforskning, målföljning och spaning. När satellitnavigeringssystem, t.ex. GPS, inte kan användas behöver plattformen istället förlita sig på sina egna sensorer för att bestämma var den är. En sådan teknik är simultaneous localization and mapping (SLAM). Ett vanligt tillvägagångssätt i SLAM är att använda sig av stillastående objekt som är lätta att känna igen, så kallade landmärken. Genom att återse dessa landmärken går det att beräkna hur mycket plattformen har rört sig. Landmärkesbaserade SLAM-metoder klarar dock inte av att positionera plattformen om den skulle befinna sig i områden utan tillräckligt informativa landmärken. Det är därför önskvärt att planera en rutt, från start till mål, som tar hänsyn till var det finns landmärken så att detta problem kan undvikas.

Rutter som är fördelaktiga ur lokaliseringssynpunkt när de utförs kan fås genom att inkludera SLAM i ruttplaneringen. Dock är det ofta inte känt i förväg vilka landmärken som kan användas, vilket försvårar situationen. Denna avhandling syftar därför till att undersöka hur landmärkesfördelningen kan användas för att förutsäga informationen från en region.

I detta arbete introduceras virtuella landmärken som ett sätt att representera information om outforskade regioner under planeringen. Dessa virtuella landmärken konstrueras för att fånga den förväntade informationen om plattformens position, baserat på sen tidigare känd landmärkestäthet. Två tillvägagångssätt för att beräkna den förväntade informationen från dessa virtuella landmärken presenteras. Det första tillvägagångssättet är utvecklat för ett allmänt fall medan det andra är ett förfinat tillvägagångssätt som utvecklats speciellt för sensorer som mäter vinkel och avstånd, såsom LiDAR-sensorer. Metoder för att generera landmärkestätheter från tidigare flygdata i ett skogsscenario presenteras också.

Vidare presenteras ett tillvägagångssätt för att beskriva sannolikheten för att detektera cylinderbaserade landmärken i LiDAR-punktmoln. Detektionssannolikheten bestäms genom att utnyttja egenskaperna hos sensorn, landmärkesdetektorn och sannolikheten för ocklusion från andra landmärken men kräver ingen exakt information om var landmärkena är. Tillvägagångsättet har utvärderats med en riktig landmärkesdetektor och simulerade LiDAR-punktmoln i ett skogsscenario.

Slutligen presenteras ett tillvägagångssätt för ruttplanering för att minimera positionsosäkerheten. Detta tillvägagångssätt utnyttjar de introducerade virtuella landmärkena, landmärkestätheter och sannolikheterna för landmärkesdetektion. Med hjälp av tillvägagångssättet kan en rutt planeras från startposition till målposition som minskar plattformens positionsosäkerhet, utan att alla landmärkens positioner behöver vara kända. 



\section{Acknowledgments}

First of all, I would like to show my most sincere appreciation to my supervisor Assoc. Prof. Daniel Axehill and co-supervisor Assoc. Prof. Gustaf Hendeby. Your support and guidance throughout this work has been invaluable. Without your help this thesis would never have happened.

I would also like to thank Jonas Nygårds for always having an optimistic view on all situations. It has always been comforting to discuss problems with you, no matter how big or small.

This thesis has been greatly improved through the help of Magnus Malmström, Anton Kullberg and Filipe "The Captain" Barbosa. I really appreciate your input and discussion. A special thanks also goes to my office-mate Filipe Barbosa. If the world was a fair place (but not necessarily a good place), everyone would have a friend like you. I hope we will be able to finish that demotivational book (that is not my thesis).

I'm also very grateful to have met and worked with Niklas Åkerblom during the last couple of years. You are an extraordinary person and I hope we will have many fruitful projects together in the future! Furthermore, I would like to thank Amanda Olmin and George Osipov for being great friends and study partners.

A big thanks goes to Johan Holmgren at SLU for interesting research discussions and for helping me understand the different aspects of forest management. I would also like to thank Per Boström-Rost for interesting discussions and inspiration and Fredrik Näsström for putting up with me even though I clearly have not given your budgets the respect they deserve. Fredrik Lantz and Jan-Erik Mathisen deserve a big thanks for being great bosses.

I am also very grateful for all the help and support I received from Martin Enqvist and Ninna Stensgård throughout my time at the Automatic control group.

I would like to thank the Swedish Defence Research Agency for giving me the opportunity to perform my research and write this thesis. I would also like to thank SecurityLink for supporting this work and WASP for letting me take part in the Affiliated PhD Student Program.

Lastly, this work would not have been possible without the tremendous support from Josefine, may the best of times still be ahead of us. Dum vivimus, vivamus!

Linköping, February 2022

Jonas Nordlöf 



\section{Contents}

Notation

xiii

1 Introduction 1

1.1 Background and motivation $\ldots \ldots \ldots \ldots \ldots \ldots \ldots$

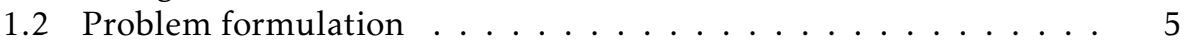

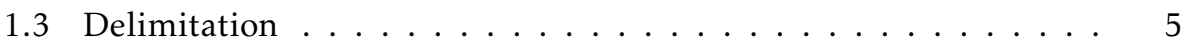

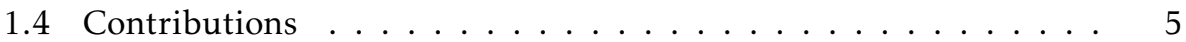

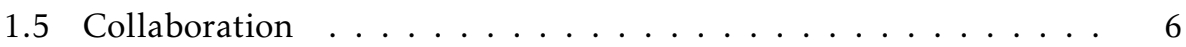

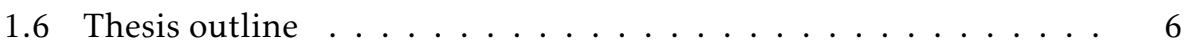

2 Background 9

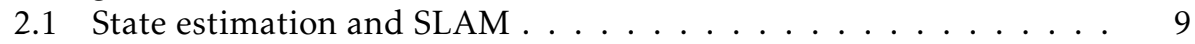

2.1 .1 State-space models . . . . . . . . . . . . . . . . 9

2.1 .2 Bayesian inference . . . . . . . . . . . . . 10

2.1 .3 Kalman filter . . . . . . . . . . . . . . . . . . . 11

2.1 .4 Information filter . . . . . . . . . . . . . . 12

2.1 .5 SLAM . . . . . . . . . . . . . . . . . . 14

2.2 Motion planning . . . . . . . . . . . . . . . 17

2.2.1 Optimization formulation . . . . . . . . . . . . 18

2.2.2 Sampling-based motion planning . . . . . . . . . . . 19

2.2 .3 Belief-space planning . . . . . . . . . . . . . . . 21

2.2 .4 Measures of optimality . . . . . . . . . . . . . 24

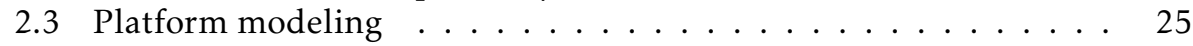

2.3 .1 Platform dynamics . . . . . . . . . . . . . 26

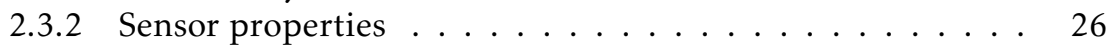

2.4 Pole-landmark detector $\ldots \ldots \ldots \ldots \ldots \ldots$

3 Virtual landmarks 31

3.1 Landmark densities . . . . . . . . . . . . . . . 31

3.1.1 Landmark density generation from forest data . . . . . 32

3.1.2 Landmark density generation from orthophotos . . . . . 33

3.2 Information gained point approximation . . . . . . . . 33

3.2.1 Subregions and virtual landmarks $\ldots \ldots \ldots \ldots$ 
3.2.2 Verifying the information approximation ....... 36

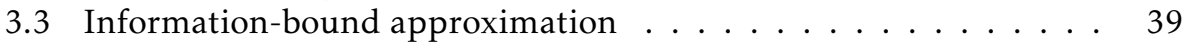

3.3.1 Information-bound update . . . . . . . . . . 40

3.3.2 Limit of true position covariance . . . . . . . . . . 42

3.4 Numerical evaluation . . . . . . . . . . . . . . . . 46

4 Landmark detection $\quad 49$

4.1 Ray-occlusion probability . . . . . . . . . . . . . 50

4.2 Derivation of $p^{\text {free }} \ldots \ldots \ldots \ldots$. . . . . . . . . . . . . . . . . 50

4.3 Estimating tree-diameter distribution . . . . . . . . . . 53

4.4 Derivation of $p_{\Omega_{i}}^{\text {size }} \ldots \ldots \ldots \ldots \ldots$. . . . . . . . . . . . . . . . . . . 54

4.5 Information estimation . . . . . . . . . . . . 56

4.6 Numerical evaluation . . . . . . . . . . . . . . . . 57

4.6.1 Simulation environment . . . . . . . . . . . 57

4.6.2 Estimated number of landmark detections . . . . . . . . . . 57

4.6.3 Effects of the probabilities $p^{\text {free }}$ and $p_{\Omega_{j}}^{\text {size }} \ldots \ldots \ldots 1$

5 Motion planning for SLAM $\quad 63$

5.1 Planning with unknown landmarks . . . . . . . . . . . 63

5.2 Numerical evaluation . . . . . . . . . . . . . . . 67

5.2.1 Implementation aspects .................. 67

5.2.2 Artificial environment ................ 68

5.2.3 Planning in a forest environment ......... 71

6 Concluding remarks $\quad 77$

6.1 Conclusion .......................... 77

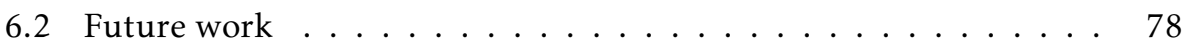

$\begin{array}{lr}\text { Bibliography } & 81\end{array}$ 
Notation

\begin{tabular}{cl} 
AbBReViations & \\
\hline Abbreviation & Meaning \\
\hline EIF & Extended information filter \\
EKF & Extended Kalman filter \\
GNSS & Global navigation satellite system \\
LIDAR & Light detection and ranging \\
MPC & Model predictive control \\
POMDP & Partially observable Markov decision process \\
RF & Radio frequency \\
SLAM & Simultaneous localization and mapping \\
SPLAM & Simultaneous planning localization and mapping \\
\hline
\end{tabular}





\section{1}

\section{Introduction}

A high positioning accuracy is often required for an autonomous system to successfully complete complex tasks. In structured environments, this is often accomplished with an artificial positioning infrastructure, such as Global Navigation Satellite Systems (GNSS), radio-frequency (RF) transmitters, WiFi, or artificial landmarks. However, attaining good positioning accuracy is much more difficult without such supporting systems

Sweden has a large proportion of its territory sparsely populated. With the exception of GNSS, there is currently little infrastructure available in these areas that can reliably be used for positioning. Furthermore, there are several situations where GNSS may be unavailable. Some of these are densely forested areas, underground mining (Nielsen, 2021) and in war zones (Angevine et al., 2019). A common technique for positioning of autonomous platforms in GNSS denied environments is to use features of the environment, such as easily recognizable trees, buildings, or any other type of landmark. Once identified, these features are used as references for computing future positions.

It is common to let the positioning systems compute a position estimate given its current information, without any possibility to influence the future motion of the platform. If the platform would move into a featureless area, such a system will not be able to generate good position estimates. It is therefore desirable to take a more active approach and let the positioning system suggest where to move the platform and what regions to avoid. To plan an informative path which takes the existence of landmarks into account, a lot of data about the position of these features is needed. This data is unrealistic to have for large and unexplored regions. Following the methodology of Box (1976), "All models are wrong, but some are useful", this thesis instead focuses on finding an approximation of this information to mitigate the problem of not having prior knowledge of these positions. 


\subsection{Background and motivation}

The problem of determining a platform's position, given observations of a known environment, is called a localization problem. Furthermore, the problem of creating a map of the environment, given the position of the platform, is referred to as a mapping problem. In simultaneous localization and mapping (SLAM) (Bailey and Durrant-Whyte, 2006; Durrant-Whyte and Bailey, 2006), neither the map nor the position of the platform is assumed to be known. Therefore, both the position and the map need to be inferred from measurements of the unknown environment and the platform's movement. There are different ways of representing the environment in a map for use in SLAM (Bresson et al., 2017; Wurm et al., 2010), one of the more common ones is landmark-based map representations. In landmark-based SLAM, the position of observed landmarks, or key-points, are stored in the map which yields a sparse representation of the environment. The type of landmark used varies depending on the application but they should be easily recognizable and distinct objects.

In most real cases, both the measurements and the platform's movement are affected by noise. Planning under such conditions is often referred to as planning under uncertainty. One of the most general formulations of planning with noisy measurements and movements, is the partially observable Markov decision process (POMDP). However, this is usually only solvable for small problems (Kaelbling et al., 1998; Papadimitriou and Tsitsiklis, 1987). Approximations are instead often introduced in order to trade optimality for computational efficiency (Bry and Roy, 2011). Instead of planning with a known platform state, such as in ordinary motion planning, a state distribution, or belief-state, is often used.

In belief-space planning, the belief-state is often estimated using state estimation techniques, such as SLAM. The specific research area of belief-space planning when both the state of the platform and the map of the area are unknown is often referred to as active SLAM, or sometimes simultaneous planning localization and mapping (SPLAM) (Labbé and Michaud, 2018). Common approaches in this research area are receding horizon control and model predictive control (MPC) (Chen et al., 2020; Indelman et al., 2015; Leung et al., 2006, 2008).

One strategy used in order to deal with the unknown future state in beliefspace planning is to disregard any new information being added to the map during the planning phase. That is, potential observations of new, previously unseen, landmarks are not taken into account when an unexplored area is observed (Chaves et al., 2014; Valencia and Andrade-Cetto, 2018). As a consequence, it is often assumed that the map is completely known a priori, as seen in (Prentice and Roy, 2009; Yang and J. Lim, 2016), or that the already explored map remains unchanged (Leung et al., 2006; Schirmer et al., 2017) during the planning phase. This leads to the situation described in Figure 1.1. In these approaches, solving the planning problem can be seen as recursively picking an area to explore and then updating the state estimate without getting new information from the environment. In a SLAM setting, since no new information, e.g., landmarks, is added to the map, this yields a conservative estimate of the information gained from exploring new areas and an overestimated position uncertainty. 


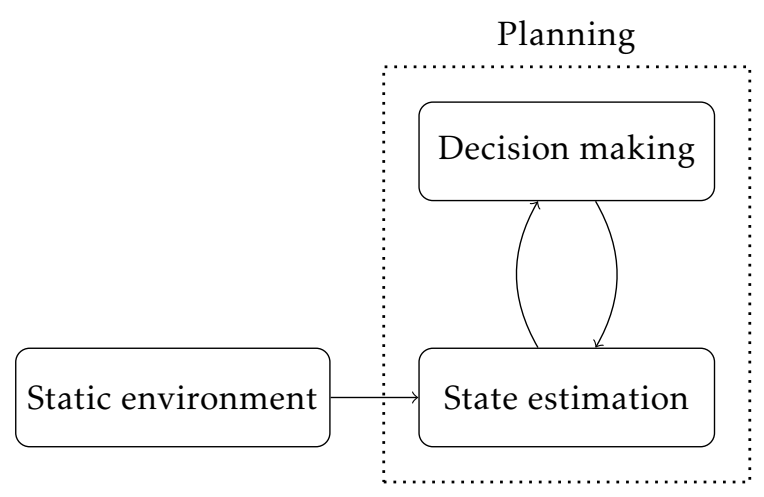

Figure 1.1: Illustration of the structure of the planning problem for minimizing the uncertainty under the assumption of a constant known map. The planning approach can be seen as several recursions of picking an area to visit and then update the state estimate given the initial map information. No new knowledge is added or gained from the environment during these recursions.

The focus in this work is to develop a representation of the map as a stochastic object describing potential and undetected landmarks, for use during planning. More specifically, the aim of this work is to develop a model for determining the expected information gained from observing a previously unexplored area without explicit knowledge of landmark positions. It is instead assumed that landmark densities, describing the spatial distribution of the landmarks' positions, are known a priori. This approach is divided into two parts, these are:

- a representation of the landmark density prior, to be used in conjunction with SLAM algorithms; and

- a model describing the probability that the landmarks are detected.

This leads to the planning procedure in Figure 1.2. In the first part, the landmark density information is represented by virtual landmarks, which fit seamlessly into SLAM algorithms. These virtual landmarks show some similarities to the measurement bundling approximation introduced in (Frey et al., 2019) for visual sensors. However, in that work, it is assumed that the measurement function is affine. Furthermore, the landmarks are not necessarily unknown and it is not shown how any environment priors should be generated. This thesis includes the generation of the landmark densities, how they can be represented by virtual landmarks and how the expected information gained from observing these can be approximated.

The probability of detecting landmarks is modeled using the introduced virtual landmarks. Pole-based landmarks and range-bearing measurements are assumed. Note that modeling the detection probability has been investigated before, see e.g., (Indelman et al., 2015; van den Berg et al., 2012). The work to 


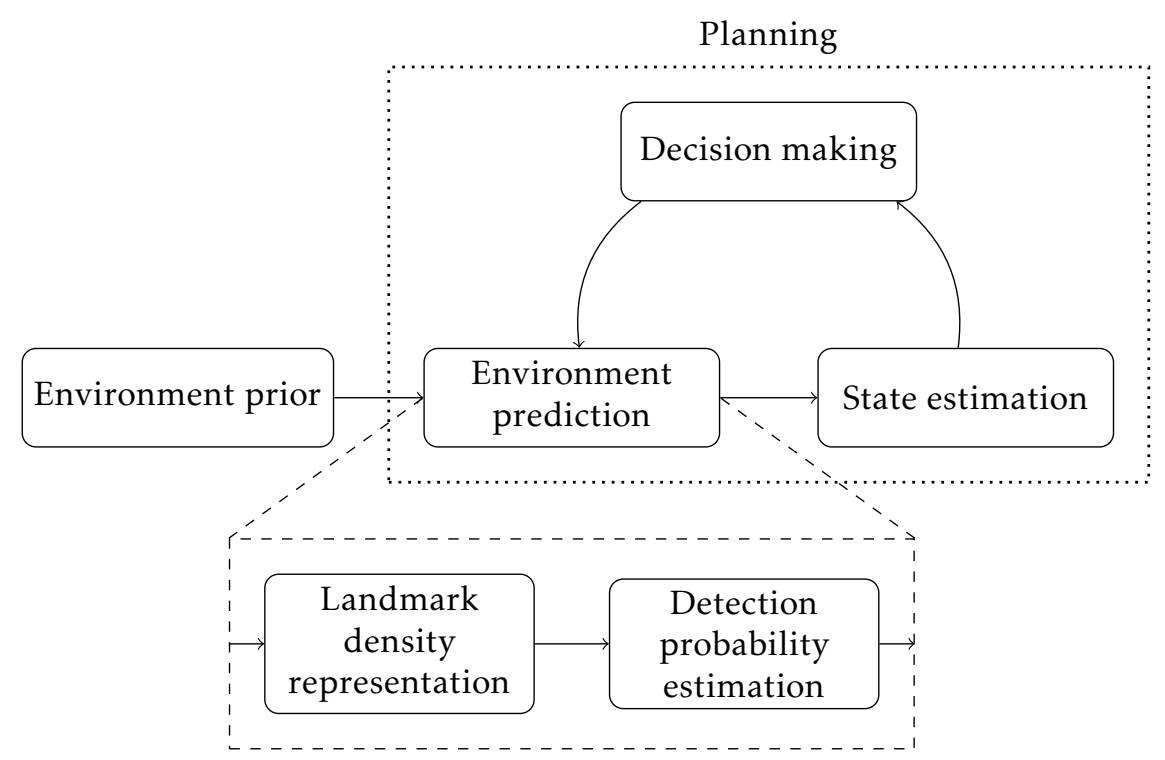

Figure 1.2: Illustration of the structure of the proposed planning problem for minimizing the uncertainty without the assumption of a constant known map. The planning approach can be seen as several recursions of picking an area to visit, predicting the new information gained from observing the area and then updating the state estimate. 
be presented goes beyond other approaches as the probability of occlusion from other landmarks is included as well as a range-dependent landmark-detection probability.

\subsection{Problem formulation}

The purpose of this work is to develop an approach for motion planning under uncertainty, where the localization during motion execution is performed using SLAM. It is assumed that there exists uncertainty in the platform's state, the motion and the measurements of the environment. Furthermore, explicit landmark positions are assumed unknown at planning time. For this situation, the aim of this thesis is to provide answers to the following research questions:

- How can the quality of the state estimates be simulated during planning, where neither all landmarks nor future measurements are known?

- How can landmark-detection probabilities be modeled and integrated in the motion-planning approach?

- How can information of landmark distributions be computed from prior data?

\subsection{Delimitation}

The focus in this work is on how to model the information gained from unexplored parts of the environment. Therefore, less focus is put on the platform motion during evaluations and only obstacle-free 2D-environments are considered. The developed methods can, to some degree, be used with any type of measurements. However, this work puts emphasis on range-bearing measurements in both the development of the methods as well as in the evaluation. Furthermore, navigation in rural and forested areas are of special interest in this work.

\subsection{Contributions}

The contributions in this thesis are presented in Chapters 3-5 and are based upon two published papers and unpublished material.

The first publication is

J. Nordlöf, G. Hendeby, and D. Axehill. Belief space planning using landmark density information. In IEEE Proceedings of the 23rd International Conference on Information Fusion (FUSION), pages 1-8, Rustenburg, South Africa, July 2020

The main contributions of this publication are a path-planning approach using landmark densities for state estimation and the introduction of the virtual landmarks used for an approximate problem formulation of the path-planning problem. The introduced virtual landmarks and how these can be integrated into 
a landmark-based SLAM approach are presented in Chapter 3, while the pathplanning approach is presented in Chapter 5 .

The second publication is

J. Nordlöf, G. Hendeby, and D. Axehill. Improved virtual landmark approximation of belief-space planning. In IEEE Proceedings of the 24th International Conference on Information Fusion (FUSION), pages 1-8, Rustenburg, South Africa, Nov. 2021

The main contributions of this publication are the introduction of an improved approach for approximating the information gained from observing an unexplored region for range-bearing measurements and a theoretical study of the properties of the derived approximation. These are presented in Chapter 3.

The thesis also includes unpublished material where the main contributions are approaches for generating landmark densities from prior aerial data in a forest scenario, presented in Chapter 3, and a model describing the landmarkdetection probabilities, presented in Chapter 4. These are combined with all previous results in an evaluation in Chapter 5.

In all of the listed contributions, the author of this thesis has driven the research, performed all experiments, and done the main part of the writing. The underlying ideas have been developed in discussions with the co-authors. The co-authors have also contributed through research discussions, technical support and help with improving the manuscripts.

\subsection{Collaboration}

The project has been partially funded by the division of C4ISR within the Swedish Defence Research Agency (FOI). The project follows the Swedish Armed Forces Research and Tech (R\&T) program for the area of autonomous systems and the area of sensors and signature management, which includes research on autonomous localization using sensor data.

\subsection{Thesis outline}

This thesis is organized as follows:

- Chapter 1 (this chapter) introduces the research problem and lists the contributions of this thesis.

- Chapter 2 outlines the theoretical foundation of SLAM and motion-planning problems. It also gives an introduction to state estimation, optimal control, and belief-space planning. Lastly, this chapter describes the platform model that is used as a basis in numerical evaluations throughout the thesis.

- Chapter 3 is based upon (Nordlöf et al., 2020, 2021) and presents two approaches for using landmark densities for estimating the future information gained from observing a region in a SLAM setting without prior knowl- 
edge of explicit landmark positions. This chapter also presents an approach for estimating landmark densities when tree stems are used.

- Chapter 4 presents a model where range-dependent landmark-detection probabilities, for pole-based landmarks and LiDAR sensors, are combined with the results from Chapter 3.

- Chapter 5 introduces a planning approach where the estimated future information is used to increase the performance of SLAM algorithms. This chapter also includes an evaluation of the methods presented in Chapter 3 and Chapter 4.

- Chapter 6 concludes the thesis and gives suggestions for future work. 



\section{2}

\section{Background}

This chapter aims at providing a theoretical background for the work presented in this thesis. In Section 2.1, the state estimation and SLAM problems are presented. This includes a review of the extended Kalman filter (EKF) and the extended information filter (EIF). Section 2.2 introduces the motion-planning problem while Section 2.3 describes the models used for evaluation in later chapters. Lastly, Section 2.4 introduces a pole-landmark detector for detecting landmarks in a LiDAR point cloud, which is also used in evaluations.

\subsection{State estimation and SLAM}

This section gives an overview of the statistical methods used to model dynamical systems and how the state is inferred from measurements.

\subsubsection{State-space models}

A state-space model is a description of a dynamical system, how it changes over time, and how measurements of the system relates to the state.

Consider an autonomous platform with the state vector $x_{t}$ and where a control input $u_{t}$ applied at time $t$ is used to steer the platform from a state $x_{t}$ to $x_{t+1}$. At each time step $t$, the state is observed through measurements $y_{t}$. In most real cases, all control inputs and measurements are affected by noise. As such, the real, exact, state $x_{t}$ is seldom known. Instead, $x_{t}$ is generally modeled by a probabilistic state-space model, where the state follows a probability distribution. Let the prior probability distribution of the initial state be denoted $p\left(x_{0}\right)$, with

$$
x_{0} \sim p\left(x_{0}\right),
$$


and assume that it is known. A general probabilistic state-space model can then be described by the conditional probability distributions (Särkkä, 2013):

$$
\begin{aligned}
x_{t+1} & \sim p\left(x_{t+1} \mid x_{t}, u_{t}\right) \\
y_{t} & \sim p\left(y_{t} \mid x_{t}\right)
\end{aligned}
$$

State-space models are by definition Markovian, i.e., given $x_{t}$, the state $x_{t+1}$ and measurements $y_{t}$ are assumed to be conditionally independent of earlier states, measurements and control inputs. This yields

$$
\begin{aligned}
p\left(x_{t+1} \mid x_{0: t}, y_{0: t}, u_{0: t}\right) & =p\left(x_{t+1} \mid x_{t}, u_{t}\right) \\
p\left(y_{t} \mid x_{0: t}, y_{0: t-1}, u_{0: t-1}\right) & =p\left(y_{t} \mid x_{t}\right),
\end{aligned}
$$

where $x_{0: t}, y_{0: t}$ and $u_{0: t}$ are used to denote the sets $\left\{x_{0}, x_{1}, \ldots, x_{t}\right\},\left\{y_{0}, y_{1}, \ldots, y_{t}\right\}$ and $\left\{u_{0}, u_{1}, \ldots, u_{t}\right\}$.

The state transition given the state $x_{t}$ and the control input $u_{t}$ in $(2.2 \mathrm{a})$ is referred to as the motion model and can be described in functional form as

$$
x_{t+1}=f\left(x_{t}, u_{t}, w_{t}\right),
$$

where $f(\cdot)$ describes the system dynamics and $w_{t}$ is the process noise. The relation between the measurements $y_{t}$ and the current state $x_{t}$, described by $(2.2 \mathrm{~b})$, is given by the measurement model, or observation model, and can similarly be represented by

$$
y_{t}=h\left(x_{t}\right)+\epsilon_{t}
$$

where $h(\cdot)$ describes the geometry of the measurements and $\epsilon_{t}$ is the measurement noise. In this work, it is assumed that $w_{t}$ and $\epsilon_{t}$ are uncorrelated zero mean Gaussian noises with covariances $Q$ and $R$.

\subsubsection{Bayesian inference}

In order to estimate the state $x_{t}$, the probability distribution

$$
p\left(x_{t+1} \mid x_{0}, y_{0: t+1}, u_{0: t}\right) \text {, }
$$

needs to be determined for all $t>0$. This problem is usually solved by a twostep recursive algorithm. The first step involves predicting the next state given the current state and applied control inputs and is referred to as the time update, formalized as

$$
p\left(x_{t+1} \mid x_{0}, y_{0: t}, u_{0: t}\right)=\int p\left(x_{t+1} \mid x_{t}, u_{t}\right) p\left(x_{t} \mid x_{0}, y_{0: t}, u_{0: t-1}\right) d x_{t} .
$$

The second step, referred to as the measurement-update, involves correcting the prediction given any available measurements using Bayes rule (Thrun et al., 2004), yielding

$$
p\left(x_{t+1} \mid x_{0}, y_{0: t+1}, u_{0: t}\right)=\frac{p\left(y_{t+1} \mid x_{t+1}\right) p\left(x_{t+1} \mid x_{0}, y_{0: t}, u_{0: t}\right)}{p\left(y_{t+1} \mid x_{0}, y_{0: t}, u_{0: t}\right)},
$$


where

$$
p\left(y_{t+1} \mid x_{0}, y_{0: t}, u_{0: t}\right)=\int p\left(y_{t+1} \mid x_{t+1}\right) p\left(x_{t+1} \mid x_{0}, y_{0: t}, u_{0: t}\right) d x_{t+1} .
$$

Recursively updating the distribution in (2.7) and (2.8) is known as Bayesian filtering (Särkkä, 2013; Thrun et al., 2004). However, computing the explicit distributions is often not possible in practice. Instead, approximations are used. Two common approximations, using a state-space model with additive Gaussian noise, are the extended Kalman filter (EKF) and the extended information filter (EIF), these are presented in the following.

\subsubsection{Kalman filter}

For the special case when $h(\cdot)$ and $f(\cdot)$ are linear and with additive noise, the state-space model can be reformulated as

$$
\begin{aligned}
x_{t+1} & =F x_{t}+G\left(u_{t}+w_{t}\right) \\
y_{t} & =H x_{t}+\epsilon_{t},
\end{aligned}
$$

where $F$ is the transition matrix, $G$ is the control-input matrix and $H$ is the measurement matrix. If the noise and the prior probability distribution are also Gaussian distributed, the Kalman filter (Kalman, 1960) provides an analytical solution for this system. Furthermore, in this setting, the distribution (2.7) after the time update and the distribution (2.8) after the measurement update are also Gaussian distributed. Since the Gaussian distribution is parameterized by its mean and covariance, these need to be propagated in both the measurement update and the time update. These steps are outlined in the following.

Let $\hat{x}_{t \mid t}$ denote the mean of this distribution at time step $t$ and let $P_{t \mid t}$ denote the corresponding covariance. The time update can then be computed as

$$
\begin{aligned}
\hat{x}_{t+1 \mid t} & =F \hat{x}_{t \mid t}+G u_{t} \\
P_{t+1 \mid t} & =F P_{t \mid t} F^{\top}+G Q G^{\top}
\end{aligned}
$$

In order to correct the prediction given the measurements, the Kalman gain is first computed. It is defined as

$$
K_{t}=P_{t \mid t-1} H^{\top}\left(H P_{t \mid t-1} H^{\top}+R\right)^{-1} .
$$

Using the Kalman gain, the mean of the distribution is then updated using the difference $\hat{\epsilon}_{t}$ between the observed measurement $y_{t}$ and the predicted measurements given the estimated state $\hat{x}_{t \mid t-1}$, yielding

$$
\begin{aligned}
\hat{\epsilon}_{t} & =y_{t}-H \hat{x}_{t \mid t-1} \\
\hat{x}_{t \mid t} & =\hat{x}_{t \mid t-1}+K_{t} \hat{\epsilon}_{t} .
\end{aligned}
$$

The covariance is then updated as

$$
P_{t \mid t}=P_{t \mid t-1}-K_{t} H P_{t \mid t-1} \text {. }
$$


Most real systems often include some types of nonlinearities, either in the motion model $f(\cdot)$ or in the measurement model $h(\cdot)$, or both. For these types of systems there exists no analytical solution in general (Thrun et al., 2004). Instead, an approximation is often introduced. In the extended Kalman filter (EKF), estimates of the state and the corresponding covariance are computed by first linearizing the motion and measurement model at the current estimate before the Kalman filter updates are applied. Thus, the true distribution is approximated by a Gaussian distribution. For this algorithm, the time update is performed as

$$
\begin{aligned}
& \hat{x}_{t+1 \mid t}=f\left(\hat{x}_{t \mid t}, u_{t}, 0\right) \\
& P_{t+1 \mid t}=F_{t} P_{t \mid t} F_{t}^{\top}+G_{t} Q G_{t}^{\top},
\end{aligned}
$$

where

$$
F_{t}=\left.\frac{\partial}{\partial x} f\left(x, u_{t}, 0\right)\right|_{x=\hat{x}_{t \mid t}}
$$

and

$$
G_{t}=\left.\frac{\partial}{\partial w} f\left(\hat{x}_{t \mid t}, u_{t}, w\right)\right|_{w=0} .
$$

Similarly, the measurement matrix $H_{t}$ is a linearization of the measurement function (2.5) and is defined as

$$
H_{t}=\left.\frac{\partial}{\partial x} h(x)\right|_{x=\hat{x}_{t \mid t-1}} .
$$

The measurement update can then be performed by updating $\hat{x}_{t \mid t}$ and $P_{t \mid t}$ as

$$
\begin{aligned}
& \hat{x}_{t \mid t}=\hat{x}_{t \mid t-1}+K_{t} \hat{\epsilon}_{t} \\
& P_{t \mid t}=P_{t \mid t-1}-K_{t} H_{t} P_{t \mid t-1},
\end{aligned}
$$

where

$$
\begin{aligned}
& \hat{\epsilon}_{t}=y_{t}-h\left(\hat{x}_{t \mid t-1}\right) \\
& K_{t}=P_{t \mid t-1} H_{t}^{\top}\left(H_{t} P_{t \mid t-1} H_{t}^{\top}+R\right)^{-1} .
\end{aligned}
$$

\subsubsection{Information filter}

An alternative formulation of the Kalman filter is the information filter. The information filter is the dual of the Kalman filter (Thrun et al., 2004) and, as such, the solutions are equivalent. While the Kalman filter uses a Gaussian distribution parameterized by its moments, the information filter uses a canonical parameterization of the Gaussian distribution. In this parameterization, an information vector $\iota_{t \mid t}$ and information matrix $\mathcal{I}_{t \mid t}$ are recursively updated instead of the mean and covariance of the distribution.

The relation between $\iota_{t \mid t}$ and $\mathcal{I}_{t \mid t}$, and $\hat{x}_{t \mid t}$ and $P_{t \mid t}$ are given by

$$
\begin{aligned}
\mathcal{I}_{t \mid t} & =P_{t \mid t}^{-1} \\
\iota_{t \mid t} & =\mathcal{I}_{t \mid t} \hat{x}_{t \mid t} .
\end{aligned}
$$


With this alternative parameterization, the update steps are formulated differently in the information filter which shifts the computational burden from the measurement update to the time update. In a general implementation of the information filter, the time update involves a matrix inversion of the entire system. However, the measurement update of the information filter only includes a summation of the previous information and the new information gained from the current measurements.

For a linear system, the time update of the information matrix and information vector are

$$
\begin{aligned}
\mathcal{I}_{t+1 \mid t} & =\left(F \mathcal{I}_{t \mid t}^{-1} F^{\top}+G Q G^{\top}\right)^{-1} \\
\iota_{t+1 \mid t} & =\mathcal{I}_{t+1 \mid t}\left(F \mathcal{I}_{t \mid t}^{-1} \iota_{t \mid t}+G u_{t}\right) .
\end{aligned}
$$

The corresponding measurement update is performed as

$$
\begin{aligned}
\mathcal{I}_{t \mid t} & =\mathcal{I}_{t \mid t-1}+H^{\top} R^{-1} H \\
\iota_{t \mid t} & =\iota_{t \mid t-1}+H^{\top} R^{-1} y_{t} .
\end{aligned}
$$

For the nonlinear case, similarly to the EKF, the extended information filter (EIF) uses a linearization in order to approximate any nonlinearity in the motion or measurement models. The time update of the EIF can be formulated as

$$
\begin{aligned}
\mathcal{I}_{t+1 \mid t} & =\left(F_{t} \mathcal{I}_{t \mid t}^{-1} F_{t}^{\top}+G_{t} Q G_{t}^{\top}\right)^{-1} \\
\iota_{t+1 \mid t} & =\mathcal{I}_{t+1 \mid t} f\left(\mathcal{I}_{t \mid t}^{-1} \iota_{t \mid t}, u_{t}, 0\right)
\end{aligned}
$$

while the measurement update of the information matrix $\mathcal{I}_{t \mid t}$ is performed as

$$
\mathcal{I}_{t \mid t}=\mathcal{I}_{t \mid t-1}+H_{t}^{\top} R^{-1} H_{t} .
$$

Using the Woodbury matrix identity (Van Loan and Golub, 1996), the Kalman gain can be rewritten as

$$
K_{t}=P_{t \mid t} H_{t}^{\top} R^{-1} \text {. }
$$

Then the measurement update of $\iota_{t \mid t}$ in the EIF can be derived from the EKF as (Kailath et al., 2000)

$$
\begin{aligned}
\iota_{t \mid t} & =P_{t \mid t}^{-1}\left(\hat{x}_{t \mid t-1}+K_{t} \hat{\epsilon}_{t}\right) \\
& =P_{t \mid t}^{-1}\left(\hat{x}_{t \mid t-1}+P_{t \mid t} H_{t}^{\top} R^{-1} \hat{\epsilon}_{t}\right) \\
& =P_{t \mid t}^{-1}\left(\hat{x}_{t \mid t-1}+P_{t \mid t} H_{t}^{\top} R^{-1}\left(y_{t}-h\left(\hat{x}_{t \mid t-1}\right)\right)\right) \\
& =\left(P_{t \mid t}^{-1}-H_{t}^{\top} R^{-1} H_{t}+H_{t}^{\top} R^{-1} H_{t}\right) \hat{x}_{t \mid t-1}+H_{t}^{\top} R^{-1}\left(y_{t}-h\left(\hat{x}_{t \mid t-1}\right)\right) \\
& =\left(P_{t \mid t}^{-1}-H_{t}^{\top} R^{-1} H_{t}\right) \hat{x}_{t \mid t-1}+H_{t}^{\top} R^{-1}\left(y_{t}-h\left(\hat{x}_{t \mid t-1}\right)+H_{t} \hat{x}_{t \mid t-1}\right) \\
& =P_{t \mid t-1}^{-1} \hat{x}_{t \mid t-1}+H_{t}^{\top} R^{-1}\left(y_{t}-h\left(\hat{x}_{t \mid t-1}\right)+H_{t} \hat{x}_{t \mid t-1}\right) \\
& =\iota_{t \mid t-1}+H_{t}^{\top} R^{-1}\left(y_{t}-h\left(\hat{x}_{t \mid t-1}\right)+H_{t} \hat{x}_{t \mid t-1}\right),
\end{aligned}
$$

where (2.25) and (2.21a) are used in the fifth equality. 


\subsubsection{SLAM}

State-space models are frequently used in SLAM, where both the trajectory of the platform and the location of detected landmarks are estimated without prior information. Instead, both the platform pose and the landmark positions are inferred from measurements of the environment and movements of the platform. Consider an autonomous platform with the state vector

$$
x_{t}=\left[\begin{array}{l}
p_{t} \\
m_{t}
\end{array}\right],
$$

where $p_{t}$ describes the pose of the platform and $m_{t}=\left[m^{1 \top}, m^{2 \top}, \ldots, m^{M_{t} \top}\right]^{\top}$ is the map at time $t$ with $M_{t}$ landmarks. In SLAM, the position of the individual landmarks are generally assumed to be time-invariant. When the EKF and EIF algorithms are used for state estimation in SLAM, these approaches are sometimes called EKF-SLAM and EIF-SLAM, respectively. Adaptations of these algorithms to a SLAM setting will now be presented.

\section{EKF-SLAM}

In a SLAM setting, the time update of the EKF-SLAM algorithm is performed as the ordinary EKF time update. However, since the number of landmarks are unknown, the size of the state vector varies depending on how many landmarks that have been detected. Furthermore, due to limited sensor range, the entire state is not observed at the same time. To account for this, the measurement update in the EKF, i.e. (2.19), is adjusted. Let $y_{t}^{i}$ denote the measurement of the $i$ th landmark $m^{i}$ and let $h^{i}\left(x_{t}\right)$ denote the corresponding measurement function, such that

$$
y_{t}^{i}=h^{i}\left(x_{t}\right)+\epsilon_{t}^{i} .
$$

Furthermore, let $\mathcal{M}_{t}=\left\{\mathcal{M}_{t}^{1}, \mathcal{M}_{t}^{2}, \cdots, \mathcal{M}_{t}^{M_{t}}\right\}$ be the set of indices of all detected landmarks at time $t$, with $M_{t}$ being the total number of detected landmarks. The linearized measurement matrix $\tilde{H}_{t}$ can then be constructed as

$$
\tilde{H}_{t}=\left[\begin{array}{c}
H_{t}^{\mathcal{M}_{t}^{1}} \\
H_{t}^{\mathcal{M}_{t}^{2}} \\
\vdots \\
H_{t}^{\mathcal{M}_{t}^{M_{t}}}
\end{array}\right],
$$

where

$$
H_{t}^{i}=\left.\frac{\partial}{\partial x} h^{i}(x)\right|_{x=\hat{x}_{t \mid t-1}} .
$$

The measurement update can then be performed by updating $\hat{x}_{t \mid t}$ and $P_{t \mid t}$ as

$$
\begin{aligned}
& \hat{x}_{t \mid t}=\hat{x}_{t \mid t-1}+\tilde{K}_{t} \tilde{\epsilon}_{t} \\
& P_{t \mid t}=P_{t \mid t-1}-\tilde{K}_{t} \tilde{H}_{t} P_{t \mid t-1},
\end{aligned}
$$


where

$$
\begin{aligned}
\tilde{\epsilon}_{t} & =\left[\begin{array}{c}
y_{t}^{\mathcal{M}_{t}^{1}}-h^{\mathcal{M}_{t}^{1}}\left(\hat{x}_{t \mid t-1}\right) \\
y_{t}^{\mathcal{M}_{t}^{2}}-h^{\mathcal{M}_{t}^{2}}\left(\hat{x}_{t \mid t-1}\right) \\
\vdots \\
\mathcal{M}_{t}^{M_{t}}-h^{\mathcal{M}_{t}^{M_{t}}}\left(\hat{x}_{t \mid t-1}\right)
\end{array}\right], \\
\tilde{K}_{t} & =P_{t \mid t-1} \tilde{H}_{t}^{\top}\left(\tilde{H}_{t} P_{t \mid t-1} \tilde{H}_{t}^{\top}+\tilde{R}_{t}\right)^{-1},
\end{aligned}
$$

and where $\tilde{R}_{t}$ is a block-diagonal matrix with $M_{t}$ instances of $R$ along the diagonal, i.e.:

$$
\tilde{R}_{t}=\left[\begin{array}{cccc}
R & 0 & \cdots & 0 \\
0 & R & \ddots & \vdots \\
\vdots & \ddots & \ddots & 0 \\
0 & \cdots & 0 & R
\end{array}\right] .
$$

A complete recursion step of the EKF-SLAM algorithm is outlined in Algorithm 2.1 .

\section{EIF-SLAM}

When using EIF in a SLAM setting, the estimates $\hat{x}_{t \mid t-1}$ and $\hat{x}_{t \mid t}$ need to be computed to perform the linearizations of the motion model and the measurement model, and for computing the measurement error. In order to avoid unnecessary inversions of the information matrix, the information vectors $\iota_{t \mid t}$ and $\iota_{t \mid t-1}$ are often replaced by $\hat{x}_{t \mid t}$ and $\hat{x}_{t \mid t-1}$ in EIF-SLAM. This is also done in the algorithm outlined below.

The time update of the EIF-SLAM algorithm can be formulated as

$$
\begin{aligned}
\hat{x}_{t+1 \mid t} & =f\left(\hat{x}_{t \mid t}, u_{t}, 0\right) \\
\mathcal{I}_{t+1 \mid t} & =\left(F_{t} \mathcal{I}_{t \mid t}^{-1} F_{t}^{\top}+G_{t} Q G_{t}^{\top}\right)^{-1} .
\end{aligned}
$$

Using (2.31), the measurement update of the information matrix $\mathcal{I}_{t \mid t}$ in EIFSLAM can be performed as

$$
\mathcal{I}_{t \mid t}=\mathcal{I}_{t \mid t-1}+\sum_{i \in \mathcal{M}_{t}} \mathcal{I}_{t}^{i} \text {, }
$$

where

$$
\mathcal{I}_{t}^{i}=H_{t}^{i \top} R^{-1} H_{t}^{i}
$$

Then the measurement update of $\hat{x}_{t \mid t}$ in EIF-SLAM algorithm can be derived from 


\section{Algorithm 2.1: EKF-SLAM}

1: Input: $\hat{x}_{t-1 \mid t-1}, P_{t-1 \mid t-1}, u_{t-1}, y_{t}=\left\{y_{t}^{i}\right\}_{i \in \mathcal{M}_{t}}$ Time update:

2: $\hat{x}_{t \mid t-1}=f\left(\hat{x}_{t-1 \mid t-1}, u_{t-1}, 0\right)$

3: $F_{t-1}=\left.\frac{\partial}{\partial x} f\left(x, u_{t-1}, 0\right)\right|_{x=x}$

4: $G_{t-1}=\frac{\partial}{\partial w} f\left(\hat{x}_{t-1 \mid t-1}, u_{t-1}, w\right) \mid$

5: $P_{t \mid t-1}=F_{t-1} P_{t-1 \mid t-1} F_{t-1}^{\top}+G_{t-1} Q G_{t-1}^{\top}$

Measurement update:

6: $H_{t}^{i}=\left.\frac{\partial}{\partial x} h^{i}(x)\right|_{x=\hat{x}_{t \mid t-1}}, \quad \forall i \in \mathcal{M}_{t}$

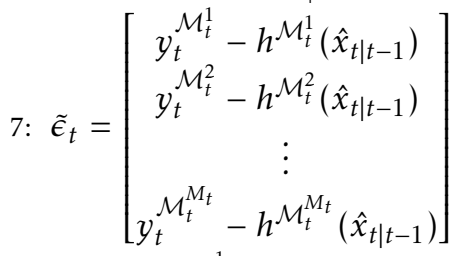

8: $\tilde{H}_{t}=\left[\begin{array}{c}H_{t}^{\mathcal{M}_{t}^{1}} \\ H_{t}^{\mathcal{M}_{t}^{2}} \\ \vdots \\ H_{t}^{\mathcal{M}_{t}^{M_{t}}}\end{array}\right]$

9: $\tilde{R}_{t}=\left[\begin{array}{cccc}R & 0 & \cdots & 0 \\ 0 & R & \ddots & \vdots \\ \vdots & \ddots & \ddots & 0 \\ 0 & \cdots & 0 & R\end{array}\right]$

10: $\tilde{K}_{t}=P_{t \mid t-1} \tilde{H}_{t}^{\top}\left(\tilde{H}_{t} P_{t \mid t-1} \tilde{H}_{t}^{\top}+\tilde{R}_{t}\right)^{-1}$

11: $P_{t \mid t}=P_{t \mid t-1}-\tilde{K}_{t} \tilde{H}_{t} P_{t \mid t-1}$

12: $\hat{x}_{t \mid t}=\hat{x}_{t \mid t-1}+\tilde{K}_{t} \tilde{\epsilon}_{t}$

13: Output: $\hat{x}_{t \mid t}, P_{t \mid t}$ 
the EKF-SLAM algorithm as

$$
\begin{aligned}
\hat{x}_{t \mid t} & =\hat{x}_{t \mid t-1}+\tilde{K}_{t} \tilde{\epsilon}_{t} \\
& =\hat{x}_{t \mid t-1}+P_{t \mid t} \tilde{H}_{t}^{\top} \tilde{R}_{t}^{-1} \tilde{\epsilon}_{t} \\
& =\hat{x}_{t \mid t-1}+\sum_{i \in \mathcal{M}_{t}} P_{t \mid t} H_{t}^{i \top} R^{-1}\left(y_{t}^{i}-h^{i}\left(\hat{x}_{t \mid t-1}\right)\right) \\
& =\hat{x}_{t \mid t-1}+\sum_{i \in \mathcal{M}_{t}} \mathcal{I}_{t \mid t}^{-1} H_{t}^{i \top} R^{-1}\left(y_{t}^{i}-h^{i}\left(\hat{x}_{t \mid t-1}\right)\right) \\
& =\mathcal{I}_{t \mid t}^{-1}\left(\mathcal{I}_{t \mid t-1}+\sum_{i \in \mathcal{M}_{t}} H_{t}^{i \top} R^{-1} H_{t}^{i}\right) \hat{x}_{t \mid t-1}+\sum_{i \in \mathcal{M}_{t}} \mathcal{I}_{t \mid t}^{-1} H_{t}^{i \top} R^{-1}\left(y_{t}^{i}-h^{i}\left(\hat{x}_{t \mid t-1}\right)\right) \\
& =\mathcal{I}_{t \mid t}^{-1}\left(\mathcal{I}_{t \mid t-1} \hat{x}_{t \mid t-1}+\sum_{i \in \mathcal{M}_{t}} H_{t}^{i \top} R^{-1}\left(y_{t}^{i}-h^{i}\left(\hat{x}_{t \mid t-1}\right)+H_{t}^{i} \hat{x}_{t \mid t-1}\right)\right),
\end{aligned}
$$

where

$$
\tilde{K}_{t}=P_{t \mid t} \tilde{H}_{t}^{\top} \tilde{R}^{-1}
$$

was used in the second equality and (2.36) was used in the fifth. A complete recursion step of the EIF-SLAM algorithm is outlined in Algorithm 2.2.

The EKF-SLAM and EIF-SLAM are equivalent methods for solving the SLAM problem. However, a benefit of using the EIF-SLAM algorithm is that it is easier to represent no information in the EIF-SLAM algorithm than infinite uncertainty in the EKF-SLAM algorithm (Gustafsson, 2010b; Thrun et al., 2004). In later chapters, the structure of the measurement update in the EIF-SLAM algorithm will be utilized to compute approximations on the information gained when observing a specific part of the map.

\subsection{Motion planning}

This section introduces the motion-planning problem and two common ways of solving it. The motion-planning problem, sometimes referred to as the piano movers problem, consists of developing a plan that moves an object through an environment in order to reach a goal state.

The criterion for success in motion planning often varies depending on the application. Most applications can be divided into two general groups of planning objectives (LaValle, 2006), these are

- Feasibility: Find a plan that moves the platform to the goal state, regardless of efficiency.

- Optimality: Find a feasible plan that moves the platform to the goal state with the lowest cost, given some performance measure.

While the feasibility problem can be hard enough to solve, the optimality criterion is often of greater interest. However, this objective comes with the added complexity of formulating a correct and computable performance measure. This will be investigated in the following. 


\section{Algorithm 2.2: EIF-SLAM}

1: Input: $\hat{x}_{t-1 \mid t-1}, \mathcal{I}_{t-1 \mid t-1}, u_{t-1}, y_{t}=\left\{y_{t}^{i}\right\}_{i \in \mathcal{M}_{t}}$ Time update:

2: $\hat{x}_{t \mid t-1}=f\left(\hat{x}_{t-1 \mid t-1}, u_{t-1}, 0\right)$

3: $F_{t-1}=\left.\frac{\partial}{\partial x} f\left(x, u_{t-1}, 0\right)\right|_{x=\hat{x}_{t-1 \mid t-1}}$

4: $G_{t-1}=\left.\frac{\partial}{\partial w} f\left(\hat{x}_{t-1 \mid t-1}, u_{t-1}, w\right)\right|_{w=0}$

5: $\mathcal{I}_{t \mid t-1}=\left(F_{t-1} \mathcal{I}_{t-1 \mid t-1}^{-1} F_{t-1}^{\top}+G_{t-1} Q G_{t-1}^{\top}\right)^{-1}$

Measurement update:

6: for $i \in \mathcal{M}_{t}$ do

7: $\quad H_{t}^{i}=\left.\frac{\partial}{\partial x} h^{i}(x)\right|_{x=\hat{x}_{t \mid t-1}}$

8: $\quad \hat{\epsilon}_{t}^{i}=y_{t}^{i}-h^{i}\left(\hat{x}_{t \mid t-1}\right)+H_{t}^{i} \hat{x}_{t \mid t-1}$

9: end for

10: $\mathcal{I}_{t \mid t}=\mathcal{I}_{t \mid t-1}+\sum_{i \in \mathcal{M}_{t}} H_{t}^{i \top} R^{-1} H_{t}^{i}$

11: $\hat{x}_{t \mid t}=\mathcal{I}_{t \mid t}^{-1}\left(\mathcal{I}_{t \mid t-1} \hat{x}_{t \mid t-1}+\sum_{i \in \mathcal{M}_{t}} H_{t}^{i \top} R^{-1} \hat{\epsilon}_{t}^{i}\right)$

12: Output: $\hat{x}_{t \mid t}, \mathcal{I}_{t \mid t}$

\subsubsection{Optimization formulation}

This section gives a brief introduction to the optimization formulation of the planning problem. For a more thorough introduction of the different concepts and methods used in optimization, the reader is referred to, e.g., (Boyd and Vandenberghe, 2004) and (Nocedal and Wright, 1999).

A mathematical optimization problem has the following general form

$$
\begin{array}{ll}
\underset{z}{\operatorname{minimize}} & J(z) \\
\text { subject to } & f_{i}(z) \leq 0, \quad i=1,2, \ldots, n .
\end{array}
$$

The vector $z=\left\{z_{1}, z_{2}, \ldots, z_{T}\right\}$ is the decision variable and the scalar-valued function $J(\cdot)$ is called the objective function or performance measure. The functions $f_{i}(\cdot), i=1,2, \ldots, n$ are the constraint functions. A vector $z$ that satisfies all constraints in (2.40) is called an optimal solution if there are no other vectors with a smaller objective value. This vector is denoted $z^{*}$.

In optimal control theory, mathematical optimization is combined with control of dynamical systems in order to compute optimal inputs $u_{0: T}$ or a policy $\pi$ such that the performance measure is minimized. Consider a platform with dynamics characterized by the motion model in (2.4) and where the state distribution is estimated using any available measurements $y_{t}^{i}$, as defined in (2.29). Denote the initial state of the platform $x_{i n i t}$ and assume that it should travel to a goal set $\mathcal{X}_{\text {goal }}$. If the noise terms $w_{t}$ and $\epsilon_{t}^{i}$ are known, in (2.4) and (2.29) respectively, this is a deterministic optimal control problem. That is, for a given state $x_{t}$ and input $u_{t}$ the future state $x_{t+1}$ can be fully determined. Since these noise 
terms are unknown in practice, this problem turns into a stochastic optimal control problem where the state $x_{t+1}$ is described by the conditional distribution in (2.6). The corresponding stochastic optimization problem can be formulated as

$$
\begin{array}{cl}
\underset{\pi, T}{\operatorname{minimize}} & \mathbb{E}\left(J\left(x_{0: T}\right)\right) \\
\text { subject to } & x_{t+1}=f\left(x_{t}, u_{t}, w_{t}\right), \\
& y_{t}^{i}=h^{i}\left(x_{t}\right)+\epsilon_{t}^{i}, \quad \forall i \in \mathcal{M}_{t}, \\
& u_{t}=\pi\left(p\left(x_{t} \mid y_{0: t}, u_{0: t-1}, x_{0}\right)\right) \\
& x_{0}=x_{\text {init }}, \\
& x_{T} \in \mathcal{X}_{\text {goal }}, \\
& \epsilon_{t}^{i} \sim \mathcal{N}(0, R), \\
& w_{t} \sim \mathcal{N}(0, Q),
\end{array}
$$

where the expectation in the objective function is evaluated with respect to the process and measurement noise as well as all possible landmark positions. Finding the optimal solution for (2.41) can in principle be done by using dynamic programming. However, this is generally intractable except for special cases (Sontag, 2013). Instead, it is often necessary to consider approximations of the problem. In Section 2.2.2, an approximation for planning with a discretized states space is presented while Section 2.2.3 presents an approximation for planning when a distribution is considered rather than a specific state.

\subsubsection{Sampling-based motion planning}

Motion-planning algorithms are generally divided into three types of categories (LaValle, 2006):

1. Complete: A complete planning algorithm finds a solution in a finite amount of time if it exists.

2. Resolution complete: A resolution complete algorithm finds a solution, in the given resolution, in a finite amount of time if it exists.

3. Probabilistic complete: The probability that a probabilistic complete algorithm finds a solution, if it exists, approaches one as the number of samples approaches infinity.

One of the most commonly used classes of motion-planning methods are the sampling-based methods. Instead of considering all possible platform states during motion planning, which is intractable in most cases, sampling-based methods only consider a subset of states based upon an implementation specific sampling scheme. Examples of such sampling schemes are randomly generated points or grid-based structures. This also means that the action space is constrained such that each possible action in a feasible position moves the platform into another feasible position. 
This sampling allows the algorithms to plan a path in large environments and makes these methods well suited for real applications. The drawback is that these algorithms only fulfill the resolution or probabilistic completeness guarantees. However, these algorithms have seen a large growth in popularity over the last decades and have solved many large, complicated, real-world problems, e.g., (Evestedt et al., 2016; Kuwata et al., 2009).

For a grid where each grid point represents a feasible platform position, the motion-planning problem turns into a discrete planning problem. As presented in (LaValle, 2006), a discrete planning problem can be formulated as:

1. A nonempty state space $\mathcal{X}$ which is a finite or countable finite set of states.

2. For each state $x_{t} \in \mathcal{X}$, there is a finite action space $\mathcal{U}\left(x_{t}\right)$.

3. A state-transition function $f(\cdot)$ that produces a state $x_{t+1}=f\left(x_{t}, u_{t}\right) \in \mathcal{X}$ for every $x_{t} \in \mathcal{X}$ and $u_{t} \in \mathcal{U}\left(x_{t}\right)$.

4. An initial state $x_{\text {init }} \in \mathcal{X}$.

5. A goal set $\mathcal{X}_{\text {goal }} \subset \mathcal{X}$.

The task of the planning algorithm is then to find a sequence of inputs $u_{0: T-1}$ such that the final state $x_{T}$ ends up in $\mathcal{X}_{\text {goal }}$, starting in $x_{0}=x_{\text {init }}$. Discrete samplingbased methods store the possible states in the environment in a graph structure. This graph is defined by an ordered pair $\mathcal{G}=\langle\mathcal{V}, \mathcal{E}\rangle$ where $\mathcal{V}$ is a set of vertices, or nodes, and $\mathcal{E}$ is a set of edges. Each state $x_{t}$ is represented and stored in a node in $\mathcal{V}$ while the motion, or action, connecting two states are represented by an edge in $\mathcal{E}$. Each of these edges is associated with a nonnegative stage cost $l(\cdot)$. For convenience, each edge will here be defined by the pair of a state $x_{t}$ and control input $u_{t}$, and where $l\left(x_{t}, u_{t}\right)$ is the cost of applying $u_{t}$ to the state $x_{t}$. Using this formulation, graph-search methods can be applied to find a path connecting the initial state and the goal set.

Two well-known graph-search methods are Dijkstra's algorithm (Dijkstra, 1959) and the $\mathrm{A}^{*}$ algorithm (Hart et al., 1968), these are presented in the following. Dijkstra's algorithm is used to find an optimal path with the lowest cost between a start node and a set of goal nodes in a graph. The total cost of a path is the sum of the stage cost $l(\cdot)$ for all edges in the path from the initial state to a goal state.

During execution of Dijkstra's algorithm, explored and unexplored nodes are stored in a priority queue $\mathcal{V}_{\text {open }}$, called the open set, and a set $\mathcal{V}_{\text {closed }}$, called the closed set. The open set includes nodes that will be visited while the closed set includes nodes that have been visited and will not be visited again. The nodes in the open set are sorted based on the cost-to-come $g_{t}$, defined as

$$
g_{t}=\sum_{\tau=0}^{t} l\left(x_{\tau}, u_{\tau}\right)=g_{t-1}+l\left(x_{t}, u_{t}\right),
$$

that is, the cost to travel from the initial state $x_{0}$ to the current state $x_{t}$, so that the node with the lowest cost is evaluated first. 
The algorithm is initialized by adding a node containing the initial state to the open set $\mathcal{V}_{\text {open }}$. The main loop then consists of first picking the node in $\mathcal{V}_{\text {open }}$ with the lowest cost. The algorithm then finds and applies all possible actions to determine the possible successor nodes to the current node. These successor nodes are sometimes referred to as child nodes. If these are not present in neither $\mathcal{V}_{\text {open }}$ nor $\mathcal{V}_{\text {closed }}$, they are added to $\mathcal{V}_{\text {open }}$. If any of them are present in $\mathcal{V}_{\text {open }}$ with a higher cost, the more expensive node in $\mathcal{V}_{\text {open }}$ is replaced by the new successor node. The current node is then moved to $\mathcal{V}_{\text {closed }}$. These steps are then repeated until the goal set is reached.

The $\mathrm{A}^{*}$ algorithm improves upon Dijkstra's algorithm by introducing a heuristic $c(\cdot)$ estimating the cost-to-go, i.e., the cost to reach the goal set $\mathcal{X}_{\text {goal }}$ from the current state $x_{t}$. Each node in the open set is then sorted based upon the sum of its cost-to-come and heuristic, instead of being sorted based only on its cost-to-come $g_{t}$, as in Dijkstra's algorithm. If the heuristic is equal to the actual cost-to-go, denoted $c^{*}$, then no unnecessary nodes would be explored (LaValle, 2006). However, having such a heuristic is not reasonable in practice. Instead a lower estimate of the true cost-to-go is used which can increase the efficiency of the search significantly if designed correctly.

In order to obtain guarantees of finding an optimal solution with the $\mathrm{A}^{*}$ graphsearch algorithm, two properties of the heuristic function are required. These are admissibility and consistency, defined as (Russell and Norvig, 2003):

- Admissible: $c\left(x_{t}\right) \leq c^{*}\left(x_{t}\right)$

- Consistent: $c\left(x_{t}\right) \leq l\left(x_{t}, u_{t}\right)+c\left(x_{t+1}\right)$

An admissible heuristic never overestimates the true cost-to-go, while a consistent heuristic never leads to a situation where the estimated path cost decreases as the area is explored. Note that a consistent heuristic is also admissible, but an admissible heuristic does not need to be consistent. The complete $A^{*}$ algorithm can be seen in Algorithm 2.3.

\subsubsection{Belief-space planning}

In belief-space planning the belief, or the distribution, of the current state is used during planning instead of the true, here unknown, state $x_{t}$ as in ordinary motion planning. An approximation often introduced to solve (2.41) is to use certaintyequivalent control, which is closely related to the separation theorem (Åström, 1970). In this method, all noise terms are replaced by their expected value (Miller et al., 2009; Platt et al., 2010). Given these expected noise terms, an optimal control input is then computed at each time step $t$. Note that for some systems, linear Gaussian systems in particular, this would yield an optimal solution. However, global optimality is not guaranteed for nonlinear systems in general. A common approach is to let the belief be represented by the estimated state $\hat{x}_{t \mid t}$ and covariance matrix $P_{t \mid t}=\mathcal{I}_{t \mid t}^{-1}$, estimated with, e.g., either the EKF-SLAM or EIF-SLAM algorithms described in Section 2.1. The performance measure $J(\cdot)$ is thereby 


\section{Algorithm 2.3: $A^{*}$ graph-search algorithm}

1: Input: An initial node $n_{\text {init }}$ containing the initial state $n_{\text {init }} \cdot x=x_{\text {init }}$ and a goal set $\mathcal{X}_{\text {goal }}$

2: $\mathcal{V}_{\text {closed }} \leftarrow \emptyset$

3: $\mathcal{V}_{\text {open }} \leftarrow \emptyset$

4: $\mathcal{V}_{\text {open }} \cdot \operatorname{push}\left(n_{\text {init }}\right)$

5: while $\mathcal{V}_{\text {open }} \neq \emptyset$ do

6: $\quad n_{\text {current }} \leftarrow \mathcal{V}_{\text {open }} \cdot \operatorname{pop}()$

7: $\quad \mathcal{V}_{\text {closed }} \cdot \operatorname{push}\left(n_{\text {current }}\right)$

8: $\quad$ if $n_{\text {current }} . x \in \mathcal{X}_{\text {goal }}$ then

9: $\quad$ return back_track $\left(n_{\text {current }}\right)$

10: end if

11: $\quad$ for $u_{t} \in \mathcal{U}\left(n_{\text {current }} . x\right)$ do

12: $\quad x_{t+1} \leftarrow f\left(n_{\text {current }} \cdot x, u_{t}\right)$

13: $\quad$ if $x_{t+1} \in \mathcal{V}_{\text {closed }}$ then

14: $\quad$ continue

15: end if

16: $\quad g_{t+1} \leftarrow n_{\text {current }} \cdot g+l\left(n_{\text {current }} . x, u_{t}\right)$

17: $\quad n_{\text {child }} \cdot x \leftarrow x_{t+1}$

18: $\quad n_{\text {child }} \cdot$ priority $\leftarrow g_{t+1}+c\left(x_{t+1}\right)$

19: $\quad n_{\text {child }} \cdot g \leftarrow g_{t+1}$

20: $\quad n_{\text {child }}$.parent $\leftarrow n_{\text {current }}$

21: $\quad$ if $x_{t+1} \notin \mathcal{V}_{\text {open }}$ then

22: $\quad \mathcal{V}_{\text {open }} \cdot \operatorname{push}\left(n_{\text {child }}\right)$

23: else

24: $\quad n^{\prime} \leftarrow \mathcal{V}_{\text {open }} \cdot \operatorname{get}\left(\hat{x}_{t+1 \mid t+1}\right)$

25: $\quad$ if $n_{\text {child }}$. priority $<n^{\prime}$. priority then

26: $\quad V_{\text {open }}$.replace $\left(n^{\prime}, n_{\text {child }}\right)$

27: $\quad$ end if

28: $\quad$ end if

29: end for

30: end while 
also defined as a function of $\hat{x}_{0: t}=\left\{\hat{x}_{0 \mid 0}, \hat{x}_{1 \mid 1}, \ldots, \hat{x}_{t \mid t}\right\}$ and $\mathcal{I}_{0: t}=\left\{\mathcal{I}_{0 \mid 0}, \mathcal{I}_{1 \mid 1}, \ldots, \mathcal{I}_{t \mid t}\right\}$. Given this performance measure, the cost-to-come $g_{t}$ can be computed as

$$
g_{t}=J\left(\hat{x}_{0: t}, \mathcal{I}_{0: t}\right)
$$

Note that, when $J(\cdot)$ is separable in time, e.g.,

$$
J\left(\hat{x}_{0: t}, \mathcal{I}_{0: t}\right)=J\left(\hat{x}_{0: t-1}, \mathcal{I}_{0: t-1}\right)+l\left(\hat{x}_{t \mid t}, \mathcal{I}_{t \mid t}\right),
$$

then $g_{t}$ can be recursively computed as

$$
g_{t}=g_{t-1}+l\left(\hat{x}_{t \mid t}, \mathcal{I}_{t \mid t}\right)
$$

as in (2.42), where $l(\cdot)$ is the stage cost as a function of $\hat{x}_{t \mid t}$ and $\mathcal{I}_{t \mid t}$.

Let $\hat{y}_{t}^{i}=h^{i}\left(\hat{x}_{t \mid t-1}\right)$ denote the expected observation of a landmark $m^{i}$. These measurements are used for state estimation during planning since the real measurements $y_{t}^{i}$ only become available during motion execution. Since the expected value of the noise realizations are used when propagating the belief-state, the measurement error in (2.38) will always be zero, with $\hat{y}_{t}^{i}$ substituting $y_{t}^{i}$. Therefore no correction of the state estimate $\hat{x}_{t \mid t}$ is performed during planning and $\hat{x}_{t \mid t}=\hat{x}_{t \mid t-1}$.

By replacing all noise terms by their expectation, the optimization problem is reformulated as

$$
\begin{array}{cl}
\underset{\pi, T}{\operatorname{minimize}} & \mathbb{E}\left(J\left(\hat{x}_{0: T}, \mathcal{I}_{0: T}\right)\right) \\
\text { subject to } & \hat{x}_{t+1 \mid t+1}=f\left(\hat{x}_{t \mid t}, u_{t}, 0\right), \\
& \hat{y}_{t}^{i}=h^{i}\left(\hat{x}_{t \mid t}\right), \quad \forall i \in \mathcal{M}_{t}, \\
& \mathcal{I}_{t+1 \mid t+1}=\Lambda\left(\mathcal{I}_{t \mid t}, \hat{y}_{t}, \hat{x}_{t \mid t}, u_{t}\right) \\
& u_{t}=\pi\left(\hat{x}_{t \mid t}, \mathcal{I}_{t \mid t}\right) \\
& \hat{x}_{0 \mid 0}=x_{i n i t}, \\
& \hat{x}_{T \mid T} \in \mathcal{X}_{\text {goal }}, \\
& \hat{x}_{t \mid t} \in \mathcal{X}, \\
& u_{t} \in \mathcal{U}\left(\hat{x}_{t \mid t}\right),
\end{array}
$$

where the expectation in the objective function is evaluated with respect to all possible landmark positions and the function $\Lambda(\cdot)$ is the information matrix update of the estimated state distribution of the EIF-SLAM algorithm, i.e., equations (2.35b) and (2.36). If the landmarks were known during planning, this would be a deterministic optimization problem which could be solved with standard methods, as presented in Section 2.2.2, where the optimal path found by the $\mathrm{A}^{*}$ algorithm is used as parameters in the policy $\pi$. However, it is assumed that the landmarks are not known during planning in the problem studied in this thesis. In the following chapters, an approach for handling these unknown landmarks is investigated. 


\subsubsection{Measures of optimality}

A scalar-valued function $J(\cdot)$ used as performance measure is minimized in (2.46). The aim of the performance measure is to reflect the task fulfillment of the platform. This performance measure typically includes multiple parts, associated with the different aspects of the mission, e.g., traveled distance, fuel consumption and position accuracy. Parts related to the state $\hat{x}_{t \mid t}$, and the information matrix $\mathcal{I}_{t \mid t}$, or covariance matrix $P_{t \mid t}=\mathcal{I}_{t \mid t}^{-1}$, are denoted $J_{\hat{x}}(\cdot)$ and $J_{\mathcal{I}}(\cdot)$ respectively. As seen in (Prentice and Roy, 2009; Schirmer et al., 2017; Yang and J. Lim, 2016), a common technique is to combine these parts with the weights $w_{\mathcal{I}}$ and $w_{\hat{x}}$, yielding

$$
J\left(\hat{x}_{0: T}, \mathcal{I}_{0: T}\right)=w_{\mathcal{I}} J_{\mathcal{I}}\left(\mathcal{I}_{0: T}\right)+w_{\hat{x}} J_{\hat{x}}\left(\hat{x}_{0: T}\right) .
$$

Note that, care must be taken when choosing $J(\cdot)$ such that the cost is nondecreasing, i.e.,

$$
J\left(\hat{x}_{0: t}, \mathcal{I}_{0: t}\right) \leq J\left(\hat{x}_{0: t+1}, \mathcal{I}_{0: t+1}\right),
$$

otherwise the assumption of nonnegative stage cost is violated and optimality cannot be guaranteed (Russell and Norvig, 2003). For common tasks, such as finding the shortest path to the goal, the choice of performance measure $J_{\hat{x}}(\cdot)$ can be trivially computed from the set of states $\hat{x}_{0: T}$. However, for the tasks of reaching a goal with the lowest possible uncertainty in the position and orientation state estimates, the choice of performance measure $J_{\mathcal{I}}(\cdot)$ is a bit more complicated. In the following, only measures related to the covariance of the position and orientation will be considered.

The most common approaches to quantifying uncertainty in SLAM are based on real, scalar functions of the covariance matrix $P_{t \mid t}^{p}$ (Rodríguez-Arévalo et al., 2018), i.e., the submatrix of $P_{t \mid t}=\mathcal{I}_{t \mid t}^{-1}$ related to the pose of the platform. The requirement of (2.48) can be satisfied by, e.g., either summing the stage cost of all time steps,

$$
J_{\mathcal{I}}\left(\mathcal{I}_{0: t}\right)=\sum_{t=1}^{T} l\left(P_{t \mid t}^{p}\right),
$$

or by only considering the maximum cost over all time steps

$$
J_{\mathcal{I}}\left(\mathcal{I}_{0: t}\right)=\max _{t} l\left(P_{t \mid t}^{p}\right)
$$

Note that, by taking the maximum over all time steps, $g_{t}$ cannot be computed recursively as defined in (2.45). Instead, $g_{t}$ can be updated as

$$
g_{t}=\max \left(l\left(P_{t \mid t}^{p}\right), g_{t-1}\right)
$$

The different choices of $l\left(P_{t \mid t}^{p}\right)$ have been well investigated (Yang et al., 2012). In the following, three popular choices are presented, these are the determinant, trace and largest eigenvalue scalarizations. 


\section{Determinant}

The determinant of the matrix $P_{t \mid t}^{p}$ is defined as

$$
\operatorname{det} P_{t \mid t}^{p}=\prod_{i=1}^{q} \lambda_{i}\left(P_{t \mid t}^{p}\right),
$$

where $\lambda_{i}\left(P_{t \mid t}^{p}\right)$ is the $i$ th eigenvalue of $P_{t \mid t}^{p}$ and $q$ is the number of rows in $P_{t \mid t}^{p}$. Minimizing the determinant, the so-called $D$-optimality criterion, also minimizes the product of the eigenvalues. The covariance matrix can be seen as an ellipsoid describing the extent of the distribution. The eigenvalues of the covariance matrix are then the semi-axes of this covariance ellipsoid. Thus, minimizing the determinant of the covariance matrix also minimizes the volume of the ellipsoid spanned by the eigenvalues.

The determinant has been shown to have several appealing mathematical properties (Pukelsheim, 2006; Rodríguez-Arévalo et al., 2018). However, there are some practical drawbacks of using the measure. If the system gets a high position accuracy in one direction, then one of the eigenvalues, hence the determinant, of the covariance matrix approaches zero. This can lead to a situation where only improvements in a single dimension are prioritized.

\section{Trace}

Minimizing the trace of the covariance matrix,

$$
\operatorname{tr} P_{t \mid t}^{p}=\sum_{i=1}^{q} \lambda_{i}\left(P_{t \mid t}^{p}\right),
$$

also minimizes the sum of the eigenvalues of $P_{t \mid t}^{p}$. This is also referred to as the $A$-optimality criterion. The trace of the covariance matrix is the expected meansquared error (MSE) of the state estimate (Yang et al., 2012). Indirectly, this also reduces the size of the covariance ellipsoid, similar to the determinant. However, since the eigenvalues are summed, instead of multiplied, the effect where only a single direction of the uncertainty ellipsoid is minimized is not as prevalent.

\section{Largest eigenvalue}

A third approach is to minimize the largest eigenvalue, the so-called E-optimality criterion. This corresponds to minimizing the largest semi-axis of the covariance ellipsoid (Yang et al., 2012).

\subsection{Platform modeling}

This section introduces the platform dynamics and measurement model used in evaluations in the following chapters. The dynamics are based upon a $2 \mathrm{D}$ unicycle model (Thrun et al., 2004). However, the presented approaches are by no 
means restricted to this choice. The measurement model is based upon a rangebearing sensor model. This model is applicable to several types of different sensors such as LiDAR, RADAR, and stereo-cameras. While parts of the presented approach are still applicable to other types of measurements, some of the material presented in later chapters have been developed especially for this model.

\subsubsection{Platform dynamics}

In a $2 \mathrm{D}$-setting, the platform pose is described by the state

$$
p_{t}=\left[\begin{array}{c}
p_{t}^{x} \\
p_{t}^{y} \\
p_{t}^{\psi}
\end{array}\right],
$$

at time $t$, where $p_{t}^{\psi}$ is the orientation of the platform and $p_{t}^{x}$ and $p_{t}^{y}$ are its $x$ and $y$-positions, respectively. The platform dynamics are based upon a unicycle model (Thrun et al., 2004) with polar inputs which consist of a rotation $\left(u_{t}^{\psi}\right)$ followed by a translation $\left(u_{t}^{\text {tran }}\right)$, according to

$$
\begin{aligned}
x_{t+1}= & f\left(x_{t}, u_{t}, w_{t}\right) \\
= & {\left[\begin{array}{c}
p_{t}^{x}+\left(u_{t}^{\operatorname{tran}}+w_{t}^{\operatorname{tran}}\right) \cos \left(p_{t}^{\psi}+u_{t}^{\psi}+w_{t}^{\psi}\right) \\
p_{t}^{y}+\left(u_{t}^{\operatorname{tran}}+w_{t}^{\operatorname{tran}}\right) \sin \left(p_{t}^{\psi}+u_{t}^{\psi}+w_{t}^{\psi}\right) \\
p_{t}^{\psi}+u_{t}^{\psi}+w_{t}^{\psi} \\
m_{t}
\end{array}\right], }
\end{aligned}
$$

where the input $u_{t}=\left[u_{t}^{\psi}, u_{t}^{\operatorname{tran}}\right]^{\top}$ is affected by process noise $w_{t}=\left[w_{t}^{\psi}, w_{t}^{\operatorname{tran}}\right]^{\top}$, with the distribution

$$
w_{t} \sim \mathcal{N}(0, Q)
$$

The relation of $p_{t}$ and $u_{t}$ is illustrated in Figure 2.1.

During planning, the state space is discretized in the $x y$-plane allowing only a subset $\mathcal{X}$ of the otherwise continuous set of positions for the platform. The discretized subspace $\mathcal{X}$, used during planning, is a uniform grid throughout this thesis.

\subsubsection{Sensor properties}

The platform is assumed to obtain measurements of nearby landmarks using an onboard range-bearing LiDAR with a limited range and $360^{\circ}$ field of view. The obtained measurements

$$
y_{t}^{i}=\left[\begin{array}{l}
y_{t}^{i, r} \\
y_{t}^{i, \theta}
\end{array}\right]
$$

to all landmarks $m^{i}=\left[m^{i, x}, m^{i, y}\right]^{\top}$ within range are the relative range $\left(y_{t}^{i, r}\right)$ and bearing $\left(y_{t}^{i, \theta}\right)$, where $m^{i, x}$ and $m^{i, y}$ are the $x$-and $y$-coordinates of the landmark. 


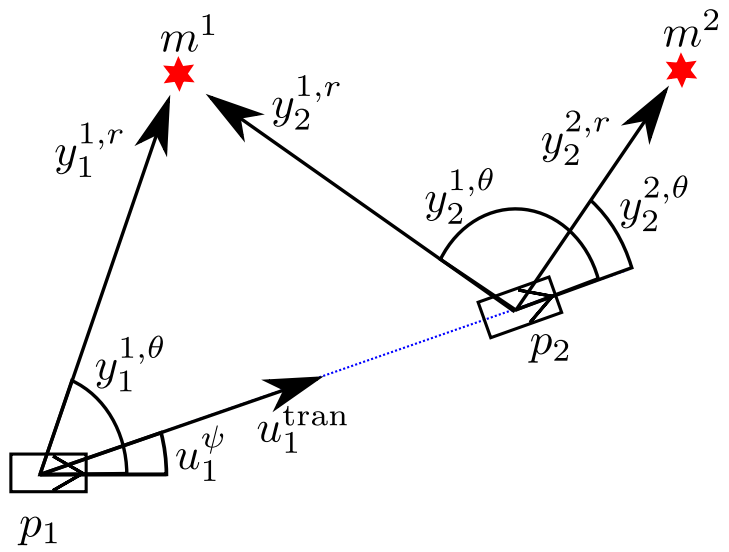

Figure 2.1: The figure shows how the positions of the platform $p_{t}$, control signals $u_{t}=\left[u_{t}^{\psi}, u_{t}^{\text {tran }}\right]^{\top}$, landmarks $m^{i}$ and measurements $y_{t}^{i}=\left[y_{t}^{i, r}, y_{t}^{i, \theta}\right]^{\top}$ relate to each other. The platform is assumed to have a sensor with limited range such that only landmark $m^{1}$ is within the sensor range from position $p_{1}$.

This gives the measurement model

$$
y_{t}^{i}=h^{i}\left(x_{t}\right)+\epsilon_{t}^{i}=\left[\begin{array}{c}
\left\|\left[p_{t}^{x}, p_{t}^{y}\right]^{\top}-m^{i}\right\| \\
\operatorname{atan} 2\left(m^{i, y}-p_{t}^{y}, m^{i, x}-p_{t}^{x}\right)-p_{t}^{\psi}
\end{array}\right]+\epsilon_{t}^{i},
$$

where $\operatorname{atan} 2(\cdot, \cdot)$ is the four-quadrant arctangent function and

$$
\epsilon_{t}^{i}=\left[\begin{array}{c}
\epsilon_{t}^{i, r} \\
\epsilon^{i, \theta}
\end{array}\right]
$$

is a vector containing the range noise $\epsilon_{t}^{i, r}$ and bearing noise $\epsilon_{t}^{i, \theta}$. The measurement noise $\epsilon_{t}^{i}$ is distributed according to

$$
\epsilon_{t}^{i} \sim \mathcal{N}(0, R) .
$$

The relation of $p_{t}, m^{i}$ and $y_{t}^{i}$ is illustrated in Figure 2.1.

\subsection{Pole-landmark detector}

The following section describes the pole-landmark detector which is developed by the Swedish Defence Research Agency and used in this thesis. The detector is used to extract detectable landmarks from the point cloud generated by the LiDAR-sensor positioned at ground level. The detector is currently used in a realtime landmark-based EKF-SLAM positioning system (Näsström et al., 2018). The detector is designed with the purpose of detecting landmarks in a point cloud 
created from a single scan of the LiDAR-sensor and not from a set of several overlapping point clouds, such as in, e.g., (Schaefer et al., 2019).

The detector works well for point clouds with different shapes and sizes. However, one requirement of the point cloud is that the points are structured into a regular grid of rows and columns, which is the case for most modern LiDAR sensors. It is also assumed that each point is generated by projecting a ray from a single origin onto the environment. This means that each column has the same azimuth angle and each row has the same elevation angle in a spherical coordinate system. Furthermore, it is assumed that there is only a single return for a specific azimuth and elevation angle. That is, an object cannot be detected behind another. For robustness, the point cloud also needs to include at least four rows of points.

A landmark is detected by first reducing the point cloud into row-wise segments of consecutive points with a small difference in radial distance to the sensor using Algorithm 2.4. Segments are then merged with other segments that are within a certain Mahalanobis distance (Mahalanobis, 1936), determined by the matrix $W$ and threshold $\varepsilon$, which have similar radial range to the sensor and horizontal position. The parameter $W$ is determined by the sensor properties, i.e., the vertical and horizontal angle resolution and the range measurement accuracy. The parameter $\varepsilon$ is determined by the landmark properties and should be similar to the landmark diameter. Note that, when merging, segments are not required to be completely overlapping. As a consequence, landmarks that are not completely vertical or straight can still be detected. Lastly, the orientation of the landmark is estimated through a principal component analysis of the points in the merged segments. This orientation is then used to find the landmark position by projecting the merged segment midpoint onto the ground plane.

In order for the merged segments to be accepted as a landmark, the following two requirements need to be fulfilled:

1. the merged segments need to include at least nine points in the four rows closest to the horizon; and

2. no object can be in front of the segments and no point in the columns to the segments left and right sides can be closer to the sensor.

Note that, as a result of the first requirement, the pole-landmark diameter needs to be large enough such that each segment can include at least $n=3$ points. An example of a detected and undetected landmark can be seen in Figure 2.2. If the merged segments fulfill these criteria, they are accepted as landmarks whereupon their ground positions are calculated. The involved steps are summarized in Algorithm 2.5. 

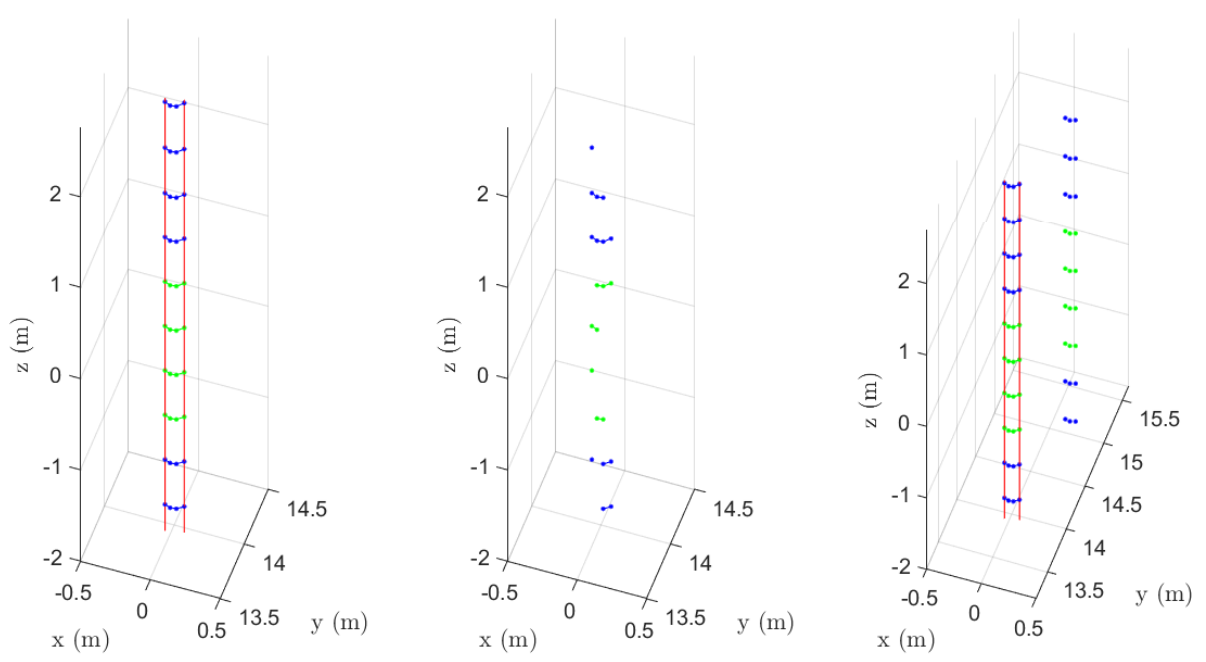

Figure 2.2: Illustration of detected and undetected landmarks. The blue and green dots represent points in the point cloud generated by a LiDARsensor positioned in the origin, the green dots mark the four rows closest to the horizon. The red lines mark the estimated edges of the detected landmarks. Left: The detected landmark fulfill the requirements of the detector, all of the four rows closest to the horizontal plane (marked in green) have included segments with more than three points. Middle: The landmark is not detected since only eight points are on the four rows closest to the horizontal plane, the first requirement for landmark detection is thereby not fulfilled. Right: Only the landmark closest to the sensor position is detected. The detector does not detect the second landmark as the first landmark is closer and directly to the left of the second landmark, the second requirement for landmark detection is thereby not fulfilled. 


\section{Algorithm 2.4: Segment extraction}

1: Input: Points $\left\{q_{i}\right\}, i \in\left\{1,2, \ldots, N_{W}\right\}$, from a single row in a point cloud with $N_{W}$ columns generated from a single revolution of a LiDAR-sensor, where $q_{i}^{x}$ and $q_{i}^{y}$ are the $x$ - and $y$-position of the point relative to the sensor origin and a threshold $\varepsilon$ defining the closeness of the points.

2: Initialization: $i \leftarrow 1, s \leftarrow 1, S_{1} \leftarrow \emptyset$

3: for $j \in\left\{2, \ldots, N_{W}\right\}$ do

4: $\quad \theta_{i, j} \leftarrow \tan ^{-1} \frac{q_{i}^{y}-q_{j}^{y}}{q_{i}^{x}-q_{j}^{x}}$

5: $\quad m^{x} \leftarrow \frac{1}{i-j} \sum_{k=i}^{j-1} q_{k}^{x}$

6: $\quad m^{y} \leftarrow \frac{1}{i-j} \sum_{k=i}^{j-1} q_{k}^{y}$

7: $\quad \delta x \leftarrow\left|\left(q_{j}^{y}-m^{y}\right) \cos \theta_{i, j}-\left(q_{j}^{x}-m^{x}\right) \sin \theta_{i, j}\right|$

8: if $\delta x>\epsilon$ then

9: $\quad s \leftarrow s+1$

10: $\quad i \leftarrow j$

11: $\quad S_{S} \leftarrow\{j\}$

12: else

13: $\quad S_{S} \leftarrow S_{S} \cup\{j\}$

14: end if

15: end for

16: Output: Segments $S_{1}, S_{2}, \ldots, S_{s}$

\section{Algorithm 2.5: Pole-landmark detector}

1: Input: Point cloud generated from a single revolution of a LiDAR-sensor.

2: Initialization: Replace all no-return points with a point at a fixed max range from the sensor.

3: Segment lines: Calculate landmark segments for the four center lines using Algorithm 2.4.

4: Merge segments: Compute the Mahalanobis distance between all segments and connect all segments below a certain threshold.

5: Segments rejection: Remove any merged segments that do not include at least nine points or has any neighboring point in front of it.

6: Orientation estimation: Estimate the orientation of the merged segments.

7: Ground projection: Project the segment midpoint on the ground using the estimated orientation.

8: Output: Return the projected segment midpoints as detected landmark positions. 


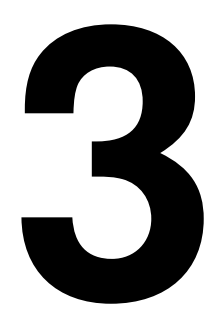

\section{Virtual landmarks}

Planning in a SLAM setting, where landmarks and future noise realizations are unknown at planning time, is a difficult problem. A common approach is to use certainty-equivalent control to deal with the unknown noise realizations, where the noise terms are replaced by their expected value. However, the problem of unknown landmarks still remains.

To overcome this problem, novel approaches for using prior estimates of the landmark densities are used in this thesis. These are presented in this chapter. Section 3.1 presents two examples of how prior estimates of the landmark densities can be generated, using aerial LiDAR data and orthophotos. Two strategies for using the landmark density for estimating the future information gained when observing a region are then introduced in Section 3.2 and Section 3.3. This is followed by a short evaluation in Section 3.4.

\subsection{Landmark densities}

In this section, two examples of how landmark densities can be estimated from publicly available aerial data for a forest scenario are presented. These densities are then, in later sections, used as a prior in order to predict the future position uncertainty of an autonomous platform. In this specific scenario it is assumed that tree stems are used as landmarks, thus trees and landmarks are used interchangeably in the following.

In the studied problem, the number of landmarks and their positions in a region of interest are unknown, let $\Omega$ denote this region. The density of landmarks is, however, assumed to be known as a function $\rho(m)$ of the position $m$ in the area, where the density describes how many landmarks are expected to be present in a position. In later sections, this density will be used to predict the expected gain in the information matrix from a discrete set of subregions, in order to take 
potential landmarks into consideration.

In the following, two different approaches for generating landmark densities are proposed based on two different datasets. These datasets are the Swedish national forest dataset (Skoglig grunddata) from Skogsstyrelsen (2021) and orthophotos from Lantmäteriet (2020).

\subsubsection{Landmark density generation from forest data}

The Swedish national forest dataset has been used in the first proposed approach for generating landmark densities. This dataset was created for use in research, forestry regulation and in the forestry industry. This dataset consists of several different variables out of which four have been used in this work. These are

- stand basal area;

- basal area-weighted mean diameter;

- basal area-weighted mean height; and

- total tree volume.

These variables are presented for square subregions of size $12.5 \times 12.5 \mathrm{~m}$ covering all of Sweden. For notational convenience, these subregions will here be denoted $\Omega_{i}$. The stand basal area and basal area-weighted mean diameter for a subregion $\Omega_{i}$ are denoted $A_{i}^{\mathrm{BA}}$ and $\bar{d}_{i}^{\mathrm{BA}}$, respectively. The basal area-weighted mean height and the total tree volume are denoted $\bar{h}_{i}^{\mathrm{BA}}$ and $V_{i}$. Using basal area-weighted variables is a common technique in the forestry industry to avoid skewed data as a result of smaller trees in the undergrowth below the tree canopy, since these trees either die or get removed during forest management. Note that the volume refers only to the accumulated stem volume of all trees in the subregion and does not include the volume of branches or canopy. The dataset is based upon LiDAR-measurements gathered during airborne surveys over multiple years. The variables are known to include errors, especially for areas with a low density where few, individual trees are standing far apart.

Using this dataset, the density can be computed from the total tree volume $V_{i}$ by dividing it with an estimated mean tree volume and the area of the subregion. This is done in the following. In order to compute the mean tree volume, it is proposed that each tree is first approximated with a cylinder. With this approximation, the estimated mean tree volume $\bar{V}_{i}$ in a subregion $\Omega_{i}$ can be computed as

$$
\bar{V}_{i}=\bar{h}_{i}^{\mathrm{BA}} \pi\left(\bar{d}_{i}^{\mathrm{BA}} / 2\right)^{2} .
$$

The estimated number of trees for the subregion, denoted $\tilde{N}_{i}$, can then be calculated by dividing the total tree volume with the mean, yielding

$$
\tilde{N}_{i}=\frac{V_{i}}{\bar{V}_{i}}=\frac{V_{i}}{\bar{h}_{i}^{\mathrm{BA}} \pi\left(\bar{d}_{i}^{\mathrm{BA}} / 2\right)^{2}} .
$$


The tree density for a subregion $\Omega_{i}$, denoted $\rho_{i}$, can then be calculated as

$$
\rho_{i}=\frac{\tilde{N}_{i}}{\left|\Omega_{i}\right|},
$$

where $\left|\Omega_{i}\right|$ is the area of the subregion. Since $\rho_{i}$ is constant over $\Omega_{i}$ when generated with this approach, it follows that

$$
\rho(m)=\rho_{i}, \quad \forall m \in \Omega_{i} .
$$

\subsubsection{Landmark density generation from orthophotos}

In the second proposed approach, landmark densities are estimated from orthophotos from (Lantmäteriet, 2020). These images have four channels, red, green, blue and infrared, with a resolution of $0.16 \mathrm{~m} /$ pixel. Each region in Sweden is updated every two to four years.

Using a visual tree detector, such as, e.g., DeepForest (Weinstein et al., 2019a,b), individual tree crowns can be detected in airborne images. DeepForest is a convolutional neural network (CNN) based single-shot detector which predicts bounding boxes over individual trees. The model is built upon the well-known RetinaNet (Lin et al., 2017) and trained using a dataset consisting of different environments and a wide range of tree species. Examples of these detections can be seen in Figure 3.1.

The landmark density can be computed by averaging the total number of detected trees over the surrounding area by using equation (3.3). The benefits of this approach are that it is good at detecting free standing trees and that the tree detections are generally accurately positioned. This makes the approach suitable for low density regions. However, the quality of the detections depends on the image resolution, time of year and time of day the image was taken. As seen in Figure 3.1, the approach is unable to detect all trees. This is especially apparent in dense forests, where it is hard to delimit trees from each other, or when trees are covered by other trees' shadows. However, as this is a learning-based approach, its performance will likely continue to improve with new algorithms and more data.

\subsection{Information gained point approximation}

The belief-state of a platform can be estimated using the EIF-SLAM algorithm as described in Section 2.1.5. However, when predicting the future state during planning, observations of new landmarks do not become available. Therefore, to take the possibility of observing new landmarks into consideration, the density of landmarks is used to predict the expected information gain of future states. In this section, a general approach for including the landmark densities in the EIF-SLAM framework is proposed. 


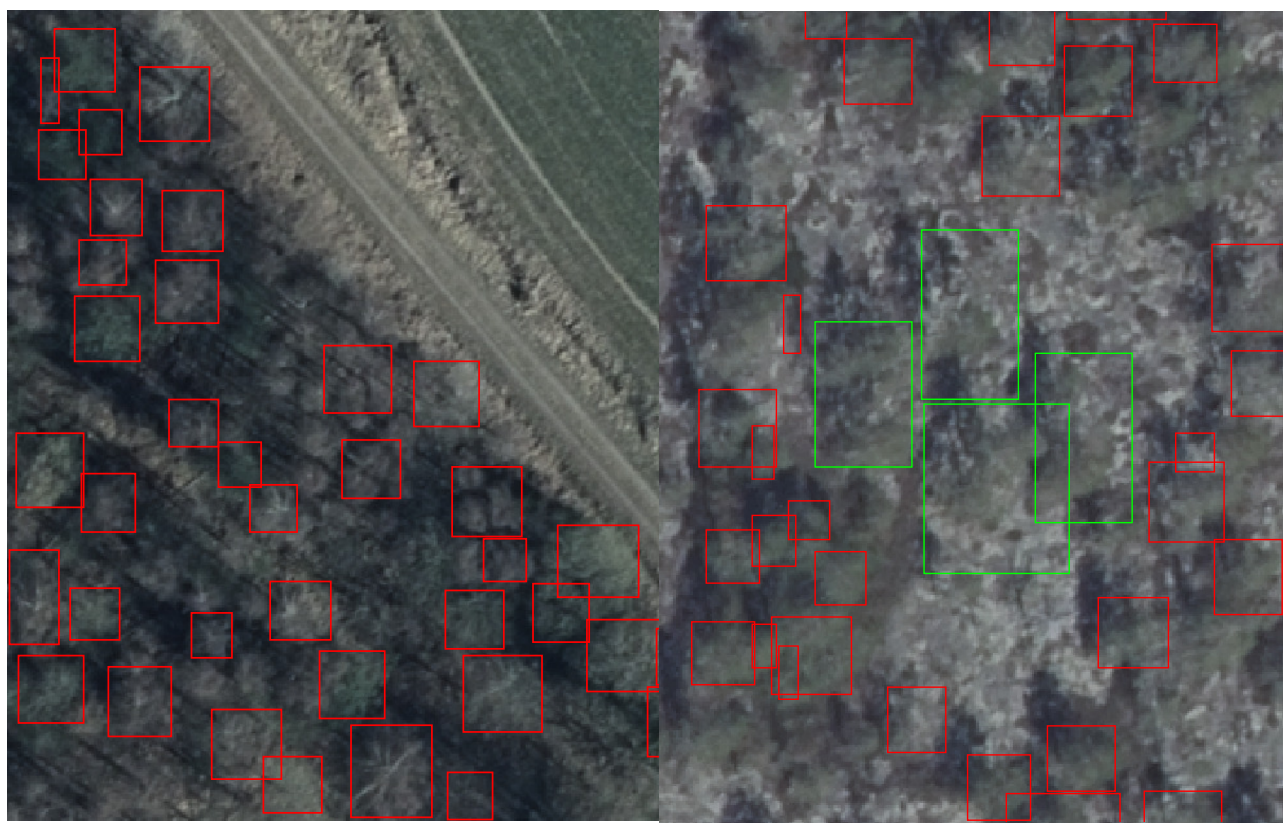

Figure 3.1: Tree detections in forested environments. Left: Detected trees are marked with a red bounding box. The position and size of the bounding boxes are accurate for all trees in the region. Right: Several trees remain undetected, here manually marked with green bounding boxes. The size of several of the detections are also too small and does not cover an entire tree. 


\subsubsection{Subregions and virtual landmarks}

For clarity, let $H_{t}\left(p_{t}, m^{i}\right)$ denote the measurement matrix $H_{t}^{i}$, in (2.31), calculated from the sensor position $p_{t}$ to a landmark at position $m^{i}$. The information gain of a single landmark at $m$ calculated from a sensor position $p_{t}$ is

$$
\mathcal{I}\left(p_{t}, m\right)=\left(H_{t}\left(p_{t}, m\right)\right)^{\top} R^{-1} H_{t}\left(p_{t}, m\right) .
$$

Now, assume the region of interest is populated with infinitely dense landmarks each with information gain

$$
d \mathcal{I}\left(p_{t}, \tilde{m}\right)=\rho(\tilde{m}) \mathcal{I}\left(p_{t}, \tilde{m}\right) d \tilde{m},
$$

where the information is compensated for the density of the landmarks by $\rho(\cdot)$. The resulting total information from the area $\Omega$ then becomes

$$
\mathcal{I}_{\Omega}\left(p_{t}\right)=\int_{\tilde{m} \in \Omega} \rho(\tilde{m}) \mathcal{I}\left(p_{t}, \tilde{m}\right) d \tilde{m} .
$$

If the density is a Dirac delta function in $m$, the result is (3.5).

Due to the lack of knowledge of future landmarks, the expression (3.7) will be used to indicate information gains. The integral is, however, not realistic to compute in practice, hence an approximation is considered. First $\Omega$ is divided into $M$ non-overlapping subregions $\Omega_{i}$, this yields

$$
\begin{aligned}
\mathcal{I}_{\Omega}\left(p_{t}\right) & =\int_{\tilde{m} \in \Omega} \rho(\tilde{m}) \mathcal{I}\left(p_{t}, \tilde{m}\right) d \tilde{m} \\
& =\sum_{i=1}^{M} \int_{\tilde{m} \in \Omega_{i}} \rho(\tilde{m}) \mathcal{I}\left(p_{t}, \tilde{m}\right) d \tilde{m} \\
& =\sum_{i=1}^{M} \mathcal{I}_{t}^{i},
\end{aligned}
$$

where

$$
\mathcal{I}_{t}^{i}=\int_{\tilde{m} \in \Omega_{i}} \rho(\tilde{m}) \mathcal{I}\left(p_{t}, \tilde{m}\right) d \tilde{m} .
$$

The integral over the subregions $\Omega_{i}$ in (3.9) could now be computed with numerical integration. However, recomputing (3.9) every time the sensor position $p_{t}$ changes is computationally expensive. Instead, if the subregions $\Omega_{i}$ are small then $\mathcal{I}\left(p_{t}, \tilde{m}\right)$ will be almost constant over $\Omega_{i}$, for a given $p_{t}$. Hence, $\mathcal{I}\left(p_{t}, \tilde{m}\right)$ in (3.9) can be approximated by $\mathcal{I}\left(p_{t}, m_{i}^{\star}\right)$ for a landmark position $m_{i}^{\star}$ in the region. This yields

$$
\mathcal{I}_{t}^{i} \approx \int_{\tilde{m} \in \Omega_{i}} \rho(\tilde{m}) \mathcal{I}\left(p_{t}, m_{i}^{\star}\right) d \tilde{m}=\left(\int_{\tilde{m} \in \Omega_{i}} \rho(\tilde{m}) d \tilde{m}\right) \mathcal{I}\left(p_{t}, m_{i}^{\star}\right) .
$$


Recomputing $\mathcal{I}\left(p_{t}, m_{i}^{\star}\right)$ is more computationally efficient than recomputing (3.9) using numerical integration when the sensor position $p_{t}$ changes. For simplicity, the center point of $\Omega_{i}$ will be used as $m_{i}^{\star}$. Note that, it is the measurement model, and its underlying geometry, that decides how large the subregions $\Omega_{i}$ can be with reasonable approximation errors. Using this approximation yields

$$
\begin{aligned}
\mathcal{I}_{\Omega}\left(p_{t}\right) & \approx \sum_{i=1}^{M}\left(\int_{\tilde{m} \in \Omega_{i}} \rho(\tilde{m}) d \tilde{m}\right) \mathcal{I}\left(p_{t}, m_{i}^{\star}\right) \\
& =\sum_{i=1}^{M} \Phi_{i} \mathcal{I}\left(p_{t}, m_{i}^{\star}\right)
\end{aligned}
$$

where cumulative densities $\Phi_{i}$ are defined as

$$
\Phi_{i}=\int_{\tilde{m} \in \Omega_{i}} \rho(\tilde{m}) d \tilde{m}
$$

and can be determined through, e.g., numerical integration. As the subregion $\Omega_{i}$ becomes smaller, the approximation improves and in the limit the left hand side and right hand side in (3.11) coincide.

The density $\rho(\cdot)$ is assumed to be constant in time and known a priori, $\Phi_{i}$ can thereby be pre-computed for all regions. On the other hand, if we assume that $\rho(\cdot)$ is constant over $\Omega_{i}$, the numerical integration of $\Phi_{i}$ in (3.12) could be replaced with a single point at, say, $m_{i}^{\star}$. However, this assumption does not necessarily hold as the density $\rho(\cdot)$ may be non-continuous or include large gradients. Since $\Phi_{i}$ can be pre-computed, the computational gain of this pointapproximation is also limited. Note that, accuracy improvements could possibly be obtained by aligning subregions with transitions in $\rho(\cdot)$ since these transitions can be large. Additional information can possibly also be extracted by utilizing these transitions. This is, however, not considered here.

The information of a subregion $\Omega_{i}$ is represented by a virtual landmark in the state when using EIF-SLAM during planning. Given a set of $M$ virtual landmarks with corresponding approximate cumulative densities $\Phi_{i}, i \in\{1,2, \ldots, M\}$, the modified measurement update of $\mathcal{I}_{t \mid t}$, in (2.36), which incorporates these approximations is

$$
\mathcal{I}_{t \mid t}=\mathcal{I}_{t \mid t-1}+\sum_{i=1}^{M} \Phi_{i} \mathcal{I}\left(p_{t}, m_{i}^{\star}\right) .
$$

This is more computationally efficient than replacing (2.36) with (3.7) directly. As the information gained from a single point is scaled to represent an entire subregion, this approach is referred to as the point-approximation approach.

\subsubsection{Verifying the information approximation}

A small evaluation of the introduced point-approximation approach is presented in this section. The purpose of this evaluation is to determine the quality of the 


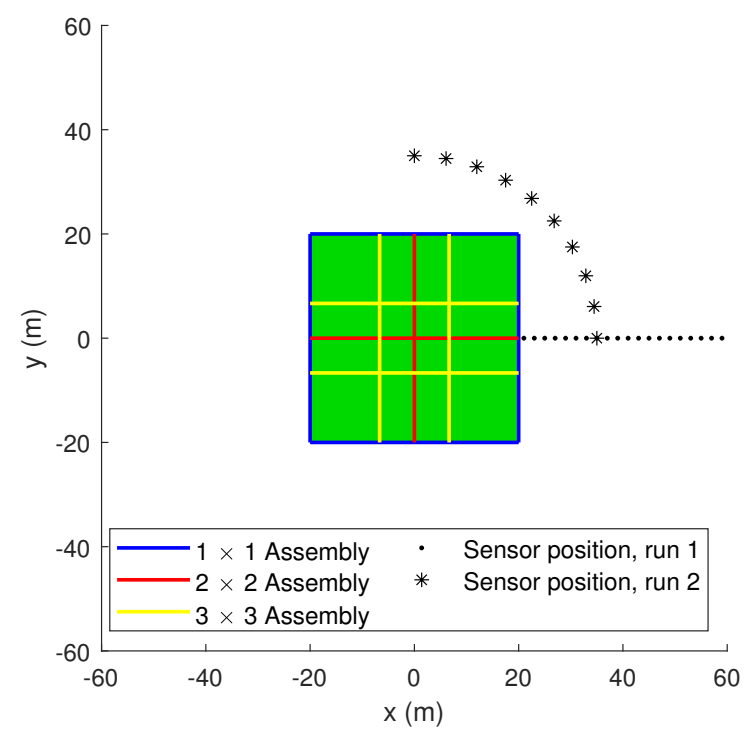

Figure 3.2: Sensor positions for model verification. The green square represents the area with a landmark density that is approximated with the three different virtual landmark sets.

information approximation introduced in (3.11), i.e. whether the center point $m_{i}^{\star}$ of each subregion approximately gives the same information as the entire subregion $\Omega_{i}$. This evaluation is based upon the measurement model presented in Section 2.3.2, where $R$, used in (3.5), is defined as

$$
R=\left[\begin{array}{cc}
0.2^{2} & 0 \\
0 & (\pi / 180)^{2}
\end{array}\right]
$$

For simplicity, the subregions $\Omega_{i}$, used for approximating the landmark density, are equally sized square regions, but, in general, any space discretization or tessellation should be applicable. Furthermore, $\Phi_{i}=1$ is used in (3.12).

The information gained from three different sets of virtual landmarks for an area $\Omega_{T}$ are compared with the integral (3.7). The area $\Omega_{T}$ is $40 \times 40 \mathrm{~m}^{2}$ and is approximated by sets of $1 \times 1,2 \times 2$ and $3 \times 3$ virtual landmarks, where each virtual landmark represents a subregion $\Omega_{i}$. The summed information gain of these sets are denoted $\mathcal{I}_{1 \times 1}^{P A}, \mathcal{I}_{2 \times 2}^{P A}$ and $\mathcal{I}_{3 \times 3}^{P A}$ respectively. An illustration of this area and the different subregions can be seen in Figure 3.2. Two different comparisons are performed. The first setup is designed to highlight the significance of the distance between the observer and the landmark region. The quality of the approximation is computed for distances between 21 and $60 \mathrm{~m}$ from the center of the landmark area, with the sensor placed on a straight line from the region midpoint. The second setup is designed to evaluate if the angle from the sensor platform to the landmark region affects the quality of the approximation. This is done with sensor locations on an arc $35 \mathrm{~m}$ from the center of the landmark area. This distance 


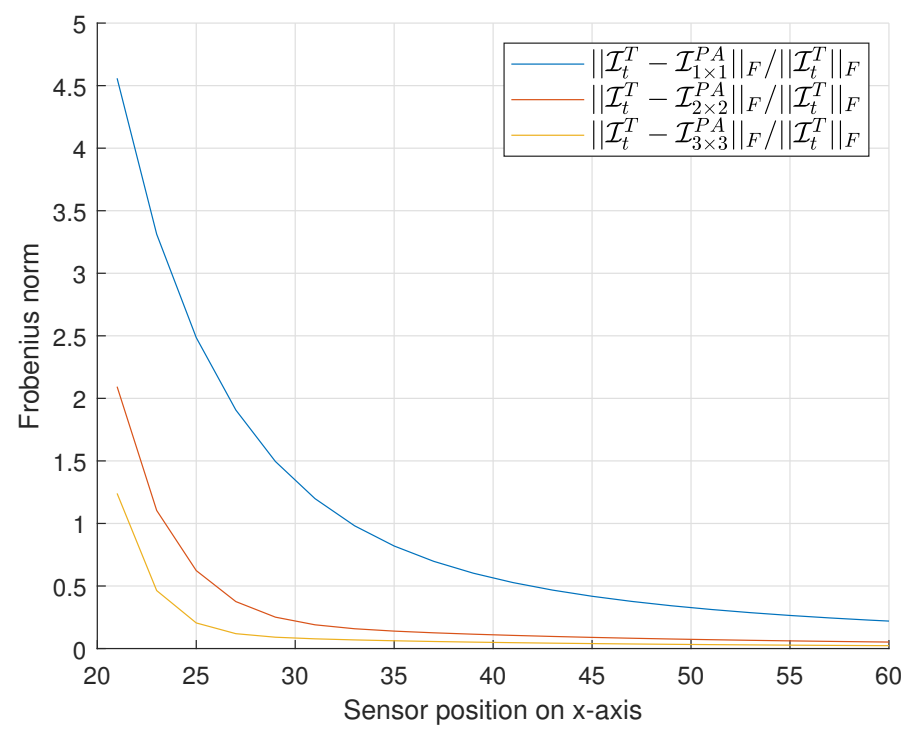

Figure 3.3: Frobenious norm of the difference between $\mathcal{I}_{t}^{T}$ and the information gain of the different landmark sets normalized by the norm of $\mathcal{I}_{t}^{T}$ with different distances to the sensor position. $\mathcal{I}_{t}^{T}$ represents the information gain computed through numerical integration, $\mathcal{I}_{1 \times 1}^{P A}, \mathcal{I}_{2 \times 2}^{P A}$ and $\mathcal{I}_{3 \times 3}^{P A}$ represents the approximate information gain computed using the point-approximation approach with the $1 \times 1,2 \times 2$ and $3 \times 3$ virtual landmark sets, respectively.

is chosen as it is considered to be on the limit of reasonable approximation errors. In both of these comparisons, the sensor is stationary and all sensor placements are evaluated independently.

For all positions, the relative error between the information matrix for the integral (3.7) and the three landmark sets are evaluated with the Frobenious norm, i.e.,

$$
\frac{\left\|\mathcal{I}_{t}^{T}-\mathcal{I}_{j \times j}^{P A}\right\|_{F}}{\left\|\mathcal{I}_{t}^{T}\right\|_{F}}, \quad j=1,2,3,
$$

where $\mathcal{I}_{t}^{T}$ is the integral (3.9) over $\Omega_{T}$. These norms can be seen in Figure 3.3 and 3.4. The information gain $\mathcal{I}_{t}^{T}$ is computed using numerical integration in Wolfram Mathematica (Wolfram Research, Inc., 2019).

The assumption that $\mathcal{I}\left(p_{t}, m_{i}\right) \approx \mathcal{I}\left(p_{t}, m_{i}^{\star}\right)$ in the entire subregion $\Omega_{i}$ is expected to be less accurate when the sensor is closer to $\Omega_{T}$. In these cases, $H_{t}\left(p_{t}, m_{i}\right)$ changes considerably within the region $\Omega_{i}$, hence the approximation $H\left(p_{t}, m_{i}\right) \approx$ $H\left(p_{t}, m_{i}^{\star}\right)$ is poor. As seen in Figure 3.3, the errors decrease with an increased distance between the sensor and the center of the region. The $2 \times 2$ and $3 \times 3$ virtual landmark sets, are considered to yield poor approximations when the sensor 


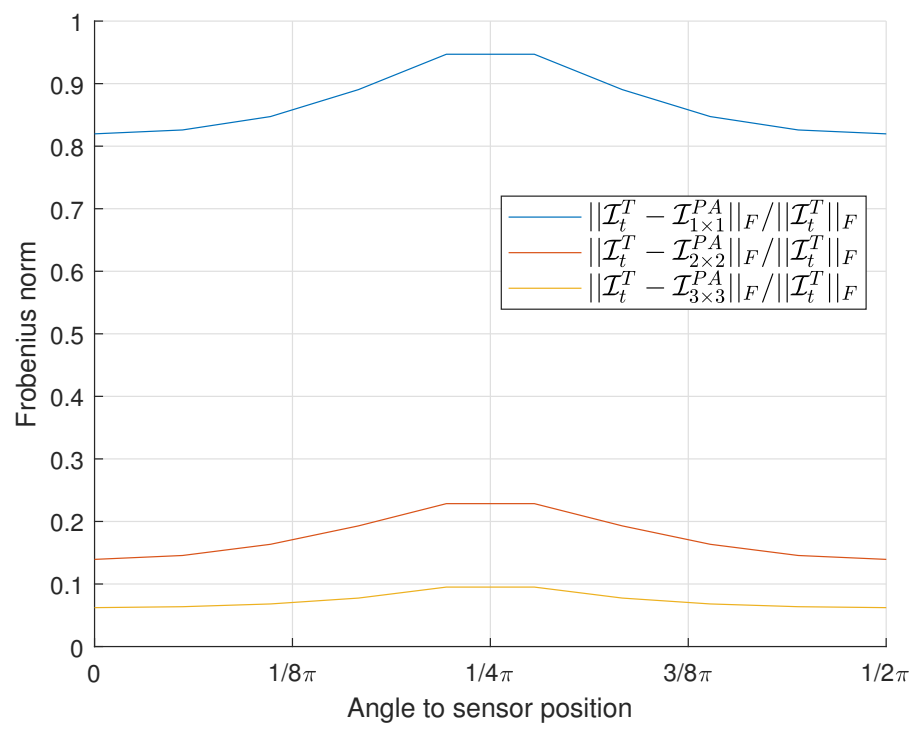

Figure 3.4: Frobenious norm of the difference between $\mathcal{I}_{t}^{T}$ and the information gain and the different landmark sets normalized by the norm of $\mathcal{I}_{t}^{T}$ at different positions around the target area. $\mathcal{I}_{t}^{T}$ represents the information gain computed through numerical integration, $\mathcal{I}_{1 \times 1}^{P A}, \mathcal{I}_{2 \times 2}^{P A}$ and $\mathcal{I}_{3 \times 3}^{P A}$ represents the approximate information gain computed using the $1 \times 1,2 \times 2$ and $3 \times 3$ virtual landmark sets, respectively.

platform is within $25 \mathrm{~m}$ of the center. The error of the $1 \times 1$ set is only considered acceptable when the sensor platform is placed at around $35 \mathrm{~m}$, or more, from the virtual landmark.

In the second comparison, seen in Figure 3.4, the error marginally increases when the sensor is close to the diagonal of the area. However, the approximation error is considered to be reasonably stable for all positions around the landmark area. That is, the accuracy is largely independent of the angle from the sensor platform to the landmark area.

\subsection{Information-bound approximation}

A key approximation in the method suggested in Section 3.2 is to use a point to represent each subregion. This approximation enables representation of the landmark densities in the EIF-SLAM algorithm. However, a single point poorly captures all of the spatial information gained from the subregion. This is especially apparent when the sensor is close to the subregion, where the approximation is the most inaccurate, as seen in Figure 3.3. Decreasing the size of the region improves the approximation, but as the complexity of the EIF-SLAM algorithm 
increases with the square of the number of subregions in the state space, this is not a computationally efficient approach. Summing the information of multiple points in a single subregion improves the approximation and the summation of infinitely dense landmarks yields an integral over $\Omega_{i}$. This approach will be pursued in the following. Range-bearing measurements are assumed.

Rather than only scaling the information matrix of a single point, a better way of representing the information matrix $\mathcal{I}_{t}^{i}$ gained from a subregion $\Omega_{i}$ is to compute the integral over $\Omega_{i}$ in (3.9) directly thereby replacing (2.37) in the EIF-SLAM algrithm with (3.9) for state estimation during the planning.

How to compute $\mathcal{I}_{t}^{i}$ depends on the shape of $\Omega_{i}$ and the measurement function $h^{i}(\cdot)$. For a range-bearing sensor, (3.9) is easily calculated for circle sectors, with the sensor position $p_{t}$ as origin. However, typically $\Omega_{i}$ is square. Instead, $\mathcal{I}_{t}^{i}$ is approximated with an upper and a lower bound, by computing the integral (3.9) over an outer and an inner approximation of $\Omega_{i}$, formed by circle sectors $\bar{\Omega}_{i, k}$ and $\underline{\Omega}_{i, k}$, as seen in Figure 3.5. Denote the maximum and minimum density in a subregion $\Omega_{i}$ as $\rho_{i}^{\max }$ and $\rho_{i}^{\min }$. Bounds on $\mathcal{I}_{t}^{i}$ can then be computed as

$$
\underline{\mathcal{I}}_{t}^{i}=\sum_{k=1}^{K} \underline{\mathcal{I}}_{t}^{i, k} \rho_{i}^{\min } \leq \mathcal{I}_{t}^{i} \leq \sum_{k=1}^{K} \overline{\mathcal{I}}_{t}^{i, k} \rho_{i}^{\max }=\overline{\mathcal{I}}_{t}^{i} \text {, }
$$

where the matrices $\underline{\mathcal{I}}_{t}^{i, k}$ and $\overline{\mathcal{I}}_{t}^{i, k}$ are defined as

$$
\begin{aligned}
& \overline{\mathcal{I}}_{t}^{i, k}=\int_{\tilde{m} \in \bar{\Omega}_{i, k}} \mathcal{I}\left(p_{t}, \tilde{m}\right) d \tilde{m} \\
& \underline{\mathcal{I}}_{t}^{i, k}=\int_{\tilde{m} \in \underline{\Omega}_{i, k}} \mathcal{I}\left(p_{t}, \tilde{m}\right) d \tilde{m} .
\end{aligned}
$$

These integrals can be efficiently computed using polar coordinates. As the number of sectors $K$ increases, the upper and lower bounds improve. In the limit, as $K \rightarrow \infty, \underline{\mathcal{I}}_{t}^{i}=\overline{\mathcal{I}}_{t}^{i}$, given $\rho_{i}^{\max }=\rho_{i}^{\text {min }}$. This approach is referred to as the information-bound approach in the following section.

\subsubsection{Information-bound update}

This section describes how the information-bound approach is integrated in the EIF-SLAM algorithm. Bounds for the collected information can be obtained using (3.16). Using $\underline{I}_{t}^{i}$ in the SLAM recursion will result in a lower bound of collected information with this subregion information approximation, where partially seen patches are dropped. Analogous, $\overline{\mathcal{I}}_{t}^{i}$ provides an upper bound, where partially seen patches are included as if fully observed. Hence, the integral approximation error can be bounded. The upper and lower bounds on $\mathcal{I}_{t \mid t}$ are denoted $\overline{\mathcal{I}}_{t \mid t}$ and $\underline{I}_{t \mid t}$, respectively. 


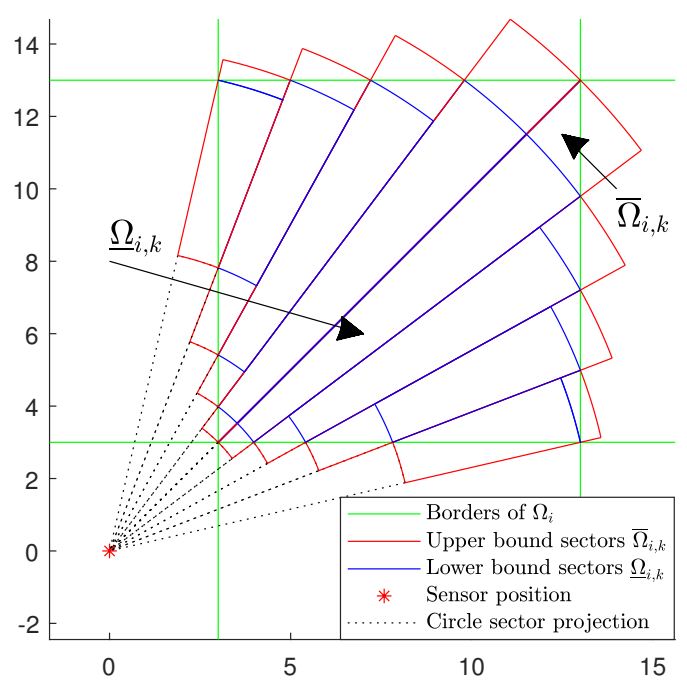

Figure 3.5: The figure shows the integrated area, in a 2D-setting, used to form the upper and lower bound of $\mathcal{I}_{t}^{i}$. The red sectors form an outer approximation of $\Omega_{i}$ and give an upper bound of $\mathcal{I}_{t}^{i}$, while the blue sectors form an inner approximation of $\Omega_{i}$ and give a lower bound.

The information time updates of the information-bounds $\overline{\mathcal{I}}_{t \mid t}$ and $\underline{\mathcal{I}}_{t \mid t}$ are equivalent to (2.35), i.e.,

$$
\begin{aligned}
& \overline{\mathcal{I}}_{t+1 \mid t}=\left(F_{t} \overline{\mathcal{I}}_{t \mid t}^{-1} F_{t}^{\top}+G_{t} Q G_{t}^{\top}\right)^{-1} \\
& \underline{\mathcal{I}}_{t+1 \mid t}=\left(F_{t} \underline{\mathcal{I}}_{t \mid t}^{-1} F_{t}^{\top}+G_{t} Q G_{t}^{\top}\right)^{-1} .
\end{aligned}
$$

To get the bound approximations, all subregions which are fully or partially within the sensor range are included in the measurement update step of the upper bound, while only the fully covered subregions are included in the measurement update of the lower bound. Denote the sets of these subregion indices as $c_{t}^{u}$ and $c_{t}^{l}$ respectively.

Using the procedure outlined above, the measurement updates of $\overline{\mathcal{I}}_{t \mid t}$ and $\underline{\mathcal{I}}_{t \mid t}$ are

$$
\begin{aligned}
& \underline{\underline{I}}_{t \mid t}=\underline{\mathcal{I}}_{t \mid t-1}+\sum_{i \in c_{t}^{l}} \underline{I}_{t}^{i}, \\
& \overline{\mathcal{I}}_{t \mid t}=\overline{\mathcal{I}}_{t \mid t-1}+\sum_{i \in c_{t}^{u}} \overline{\mathcal{I}}_{t}^{i} .
\end{aligned}
$$

If $\overline{\mathcal{I}}_{t \mid t}$ and $\underline{\mathcal{I}}_{t \mid t}$ are invertible, it follows that

$$
\begin{aligned}
& \overline{\mathcal{I}}_{t \mid t} \geq \mathcal{I}_{t \mid t} \Leftrightarrow \underline{P}_{t \mid t}=\overline{\mathcal{I}}_{t \mid t}^{-1} \leq \mathcal{I}_{t \mid t}^{-1}=P_{t \mid t}, \\
& \underline{\mathcal{I}}_{t \mid t} \leq \mathcal{I}_{t \mid t} \Leftrightarrow \bar{P}_{t \mid t}=\underline{\mathcal{I}}_{t \mid t}^{-1} \geq \mathcal{I}_{t \mid t}^{-1}=P_{t \mid t},
\end{aligned}
$$


where $P_{t \mid t}=\mathcal{I}_{t \mid t}^{-1}$ is the covariance matrix related to the state estimate, and $\bar{P}_{t \mid t}$ and $\underline{P}_{t \mid t}$ are upper and lower bounds, respectively, of the covariance matrix $P_{t \mid t}$ computed using the integral approximation (3.9). Since all square submatrices on the diagonal of a positive definite matrix are also positive definite, it also holds that $\underline{P}_{t \mid t}^{p} \leq P_{t \mid t}^{p} \leq \bar{P}_{t \mid t}^{p}$, where $P_{t \mid t}^{p}$ is the submatrix of $P_{t \mid t}$ related to the platform position and orientation estimates. Note that, since the geometries of $\Omega_{i, k}$ changes with $p_{t}$, the subregions $\Omega_{i}$ are still represented by one virtual landmark entry in $\overline{\mathcal{I}}_{t \mid t}$ and $\underline{I}_{t \mid t}$ and not one per subregion sector $\Omega_{i, k}$.

\subsubsection{Limit of true position covariance}

In addition to the approximation using upper and lower bounds on the information, as discussed in Section 3.3.1, another approximation emerges from the use of (3.9).

To represent all possible, but unknown, landmarks in a subregion, the dimension of the state space needs to be infinitely large. In the information-bound approach, this infinite set of possible landmarks is replaced by a single entry in the state space. This is in itself an approximation.

If the relative position of two landmarks is completely known, then observations of one of the landmarks will give information of both. If these landmarks were used in EIF-SLAM, they can be represented by a single entry in the information matrix. Similarly, when the information of all possible landmarks positions in a subregion $\Omega_{i}$ are represented by a single entry in the information matrix, the integral approximation in (3.9) implicitly assumes that the relative position between all points in $\Omega_{i}$ is known. This adds more information as compared to having separate points where there is uncertainty in the relative position. Thus, substituting infinitely many possible landmarks in the subregion with one landmark providing the information in (3.9) underestimates the expected covariance of the platform position and orientation estimates. This will now be studied in more detail.

Let $m^{i}$ be a point in the subregion $\Omega_{i}$. The measurement and time update of the information matrix $\mathcal{I}_{t \mid t}$ describing the state $x_{t}=\left[p_{t}^{\top}, m^{i \top}\right]^{\top}$ are given by (2.35b) and (2.36), where (3.9) is used in the latter.

\section{Augmented state space}

An auxiliary variable $\Delta^{i, j}$ is now introduced, representing the relation between $\tilde{m}^{i, j}$ and $m^{i}$. This variable creates a structure in the state space which simplifies the calculations that follow. Consider a set of possible landmarks $\left\{\tilde{m}^{i, j}\right\}_{j=1}^{M}$ in $\Omega_{i}$, and introduce the relation $\tilde{m}^{i, j}=m^{i}+\Delta^{i, j}$. For notational convenience, assume that we only have one subregion, thus the $i$-superscript can be removed. The extension to multiple subregions is trivial. The relation between $m$ and $\Delta^{j}$ can be seen in Figure 3.6. Let $\Delta=\left[\Delta^{1 \top}, \Delta^{2 \top}, \ldots, \Delta^{M \top}\right]^{\top}$ be included in the state $\tilde{x}_{t}=\left[p_{t}^{\top}, m^{\top}, \Delta^{\top}\right]^{\top}=\left[x_{t}^{\top}, \Delta^{\top}\right]^{\top}$, that is $\tilde{x}_{t}$ is formed by extending $x_{t}$ with $\Delta$. Let 


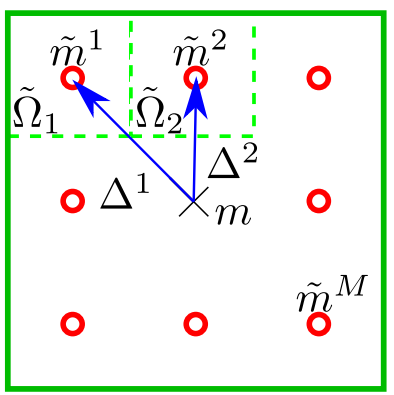

Figure 3.6: The figure shows the relation between $m, \Delta^{j}, \tilde{m}^{j}$ and $\tilde{\Omega}_{j}$ in Section 3.3.2. The boundaries of $\Omega$ are marked in green.

$\tilde{\mathcal{I}}_{t \mid t}$ denote the information of the augmented state, and partition it as

$$
\tilde{\mathcal{I}}_{t \mid t}=\left[\begin{array}{cc}
\tilde{\mathcal{I}}_{t \mid t}^{x} & \tilde{\mathcal{I}}_{t \mid t}^{x \Delta} \\
\tilde{\mathcal{I}}_{t \mid t}^{x \Delta \top} & \tilde{\mathcal{I}}_{t \mid t}^{\Delta}
\end{array}\right],
$$

where the submatrix $\tilde{\mathcal{I}}_{t \mid t}^{x}$ represents the information about the state $x_{t}=\left[p_{t}^{\top}, m^{\top}\right]^{\top}$ and $\tilde{\mathcal{I}}_{t \mid t}^{\Delta}$ represents the information about the relative position of the landmarks within $\Omega$. Similar partitions of matrices, such that their upper left submatrix is identical to their non-augmented counterpart, will be a recurring approach in the following.

The linearised dynamics for the state $\tilde{x}_{t}$ in the augmented system are $\tilde{x}_{t+1}=$ $\tilde{F}_{t} \tilde{x}_{t}+\tilde{G}_{t} w_{t}$, where $\operatorname{cov}\left(w_{t}\right)=Q$ and the matrices $\tilde{F}_{t}$ and $\tilde{G}_{t}$ are defined as

$$
\tilde{F}_{t}=\left[\begin{array}{cc}
F_{t} & 0 \\
0 & \mathbf{I}_{M \times M}
\end{array}\right]
$$

and

$$
\tilde{G}_{t}=\left[\begin{array}{c}
G_{t} \\
0
\end{array}\right],
$$

where $\mathbf{I}_{M \times M}$ is a square identity matrix with dimensions matching $\Delta$. That is, the landmarks' relative positions found in the lower block are assumed constant, hence the process noise does not increase their corresponding uncertainty. This structure will prove useful later. Note that this is a very reasonable assumption since all landmark positions are usually time invariant in SLAM.

\section{Induction basis and hypothesis}

In order to show that the approximation underestimates the real covariance of the platform position and orientation estimates, it is first shown that the upper left submatrix of $\tilde{\mathcal{I}}_{t \mid t}$ is equal to $\mathcal{I}_{t \mid t}$. This is shown by induction. Before any measurements have been obtained, it holds that

$$
\tilde{\mathcal{I}}_{0 \mid 0}^{x}=\mathcal{I}_{0 \mid 0},
$$


as no information of $m$ is available and the information about $p_{t}$ is the same in both. Next, assume that

$$
\tilde{\mathcal{I}}_{t \mid t}^{x}=\mathcal{I}_{t \mid t} .
$$

It will be shown that this assumption also holds in $t+1$.

\section{Time update}

To compute $\tilde{\mathcal{I}}_{t+1 \mid t}, \tilde{P}_{t \mid t}=\tilde{\mathcal{I}}_{t \mid t}^{-1}$ is first computed. The covariance matrix $\tilde{P}_{t \mid t}$ is then propagated before $\tilde{\mathcal{I}}_{t+1 \mid t}=\tilde{P}_{t+1 \mid t}^{-1}$ is determined. Using the matrix inversion lemma (Van Loan and Golub, 1996) and the assumption in (3.25), the submatrices of $\tilde{P}_{t \mid t}$ can be calculated as

$$
\begin{aligned}
\tilde{P}_{t \mid t}^{x} & =\mathcal{I}_{t \mid t}^{-1}+\mathcal{I}_{t \mid t}^{-1} \tilde{\mathcal{I}}_{t \mid t}^{x \Delta} \tilde{M}^{-1} \tilde{\mathcal{I}}_{t \mid t}^{x \Delta \top} \mathcal{I}_{t \mid t}^{-1} \\
\tilde{P}_{t \mid t}^{x \Delta} & =-\mathcal{I}_{t \mid t}^{-1} \tilde{\mathcal{I}}_{t \mid t}^{x \Delta} \tilde{M}^{-1} \\
\tilde{P}_{t \mid t}^{\Delta} & =\tilde{M}^{-1},
\end{aligned}
$$

where

$$
\tilde{M}=\tilde{\mathcal{I}}_{t \mid t}^{\Delta}-\tilde{\mathcal{I}}_{t \mid t}^{x \Delta \top} \mathcal{I}_{t \mid t}^{-1} \tilde{\mathcal{I}}_{t \mid t}^{x \Delta} \geq 0
$$

and

$$
\tilde{P}_{t \mid t}=\left[\begin{array}{cc}
\tilde{P}_{t \mid t}^{x} & \tilde{P}_{t \mid t}^{x \Delta} \\
\tilde{P}_{t \mid t}^{x \Delta \top} & \tilde{P}_{t \mid t}^{\Delta}
\end{array}\right]
$$

The covariance matrix after the time update is

$$
\tilde{P}_{t+1 \mid t}=\tilde{F}_{t} \tilde{\mathcal{I}}_{t \mid t}^{-1} \tilde{F}_{t}^{\top}+\tilde{G}_{t} Q \tilde{G}_{t}^{\top}
$$

with the submatrices

$$
\begin{aligned}
& \tilde{P}_{t+1 \mid t}^{x}=F_{t} \mathcal{I}_{t \mid t}^{-1} F_{t}^{\top}+G_{t} Q G_{t}^{\top}+F_{t} \mathcal{I}_{t \mid t}^{-1} \tilde{\mathcal{I}}_{t \mid t}^{x \Delta} \tilde{M}^{-1} \tilde{\mathcal{I}}_{t \mid t}^{x \Delta \top} \mathcal{I}_{t \mid t}^{-1} F_{t}^{\top}, \\
& \tilde{P}_{t+1 \mid t}^{x \Delta}=-F_{t} \mathcal{I}_{t \mid t}^{-1} \tilde{\mathcal{I}}_{t \mid t}^{x \Delta} \tilde{M}^{-1}, \\
& \tilde{P}_{t+1 \mid t}^{\Delta}=\tilde{M}^{-1} .
\end{aligned}
$$

Using the matrix inversion lemma, the upper left submatrix $\tilde{\mathcal{I}}_{t+1 \mid t}^{x}$ of the information matrix $\tilde{\mathcal{I}}_{t+1 \mid t}=\tilde{P}_{t+1 \mid t}^{-1}$ is

$$
\begin{aligned}
\tilde{\mathcal{I}}_{t+1 \mid t}^{x} & =\left(F_{t} \mathcal{I}_{t \mid t}^{-1} F_{t}^{\top}+G_{t} Q G_{t}^{\top}+F_{t} \mathcal{I}_{t \mid t}^{-1} \mathcal{I}_{t \mid t}^{x \Delta} \tilde{M}^{-1} \mathcal{I}_{t \mid t}^{x \Delta \top} \mathcal{I}_{t \mid t}^{-1} F_{t}^{\top}\right. \\
& \left.-F_{t} \mathcal{I}_{t \mid t}^{-1} \tilde{\mathcal{I}}^{x \Delta} \tilde{M}^{-1} \tilde{M} \tilde{M}^{-1} \tilde{\mathcal{I}}_{t \mid t}^{x \Delta \top} \mathcal{I}_{t \mid t}^{-1} F_{t}^{\top}\right)^{-1} \\
& =\left(F_{t} \mathcal{I}_{t \mid t}^{-1} F_{t}^{\top}+G_{t} Q G_{t}^{\top}\right)^{-1}=\mathcal{I}_{t+1 \mid t} .
\end{aligned}
$$

In conclusion, the upper left submatrix of $\tilde{\mathcal{I}}_{t+1 \mid t}$ is equal to $\mathcal{I}_{t+1 \mid t}$ after the time update. 


\section{Measurement update}

For a set of landmarks $\left\{\tilde{m}^{j}\right\}_{j=1}^{M}$ for which it holds that $\tilde{m}^{j}=m+\Delta^{j}$, (3.9) turns into

$$
\mathcal{I}_{t}=\sum_{j=1}^{M} \rho\left(m+\Delta^{j}\right) H_{t}^{j \top} R^{-1} H_{t}^{j}\left|\tilde{\Omega}_{j}\right|,
$$

where $H_{t}^{j}=\left[H_{t}^{j, p}, H_{t}^{j, m}\right], H_{t}^{j, p}$ and $H_{t}^{j, m}$ are the gradients of the measurement function with respect to $p_{t}$ and $m$ respectively, evaluated in $p_{t}$ and $m+\Delta^{j}$, and $\left|\tilde{\Omega}_{j}\right|$ is the area of the subregion associated with $\tilde{m}^{j}$. The gain in the information matrix from the measurements for the augmented state is

$$
\tilde{\mathcal{I}}_{t}=\sum_{j=1}^{M} \rho\left(m+\Delta^{j}\right) \tilde{H}_{t}^{j \top} R^{-1} \tilde{H}_{t}^{j}\left|\tilde{\Omega}_{j}\right|,
$$

where $\tilde{H}_{t}^{j}=\left[H_{t}^{j, p}, H_{t}^{j, m}, H_{t}^{j, \Delta}\right]$, and $H_{t}^{j, \Delta}$ is the gradient of the measurement function with respect to $\Delta^{j}$, evaluated in $p_{t}$ and $m+\Delta^{j}$. Since the first elements of $\tilde{H}_{t}^{j}$ are equal to $H_{t}^{j}$, the upper left submatrix of $\tilde{H}_{t}^{j \top} R^{-1} \tilde{H}_{t}^{j}$ is equal to $H_{t}^{j \top} R^{-1} H_{t}^{j}$. Hence, $\tilde{\mathcal{I}}_{t}$ can be partitioned as

$$
\tilde{\mathcal{I}}_{t}=\left[\begin{array}{cc}
\mathcal{I}_{t} & \tilde{\mathcal{I}}_{t}^{x \Delta} \\
\tilde{\mathcal{I}}_{t}^{x \Delta \top} & \tilde{\mathcal{I}}_{t}^{\Delta}
\end{array}\right] .
$$

The information after the measurement update is then

$$
\begin{aligned}
\tilde{\mathcal{I}}_{t+1 \mid t+1} & =\tilde{\mathcal{I}}_{t+1 \mid t}+\tilde{\mathcal{I}}_{t} \\
& =\left[\begin{array}{cc}
\mathcal{I}_{t+1 \mid t}+\mathcal{I}_{t} & \tilde{\mathcal{I}}_{t+1 \mid t}^{x \Delta}+\tilde{\mathcal{I}}_{t}^{x \Delta} \\
\tilde{\mathcal{I}}_{t+1 \mid t}^{x \Delta \top}+\tilde{\mathcal{I}}_{t}^{x \Delta \top} & \tilde{\mathcal{I}}_{t+1 \mid t}^{\Delta}+\tilde{\mathcal{I}}_{t}^{\Delta}
\end{array}\right] .
\end{aligned}
$$

Here, (3.31) has been used in the upper left submatrix of (3.35). In conclusion, from (3.35) it follows that this submatrix is identical to $\mathcal{I}_{t+1 \mid t+1}$ after the measurement update. By combining the results for the time update and the measurement update, the assumption introduced in (3.25) is thereby shown to also hold in $t+1$. Given the starting information (3.24), by induction, (3.25) holds for all $t$.

\section{Position covariance inequality}

Applying the matrix inversion lemma on $\tilde{\mathcal{I}}_{t \mid t}$, with respect to the upper left submatrix, yields

$$
\begin{aligned}
\tilde{P}_{t \mid t}^{x} & =\left(\tilde{\mathcal{I}}_{t \mid t}^{x}\right)^{-1}+\left(\tilde{\mathcal{I}}_{t \mid t}^{x}\right)^{-1} \tilde{\mathcal{I}}_{t \mid t}^{x \Delta} \tilde{M}^{-1} \tilde{\mathcal{I}}_{t \mid t}^{x \Delta \top}\left(\tilde{\mathcal{I}}_{t \mid t}^{x}\right)^{-1} \\
& \geq\left(\tilde{\mathcal{I}}_{t \mid t}^{x}\right)^{-1}=\mathcal{I}_{t \mid t}^{-1}=P_{t \mid t}
\end{aligned}
$$

since $\left(\tilde{\mathcal{I}}_{t \mid t}^{x}\right)^{-1} \tilde{\mathcal{I}}_{t \mid t}^{x \Delta} \tilde{M}^{-1} \tilde{\mathcal{I}}_{t \mid t}^{x \Delta \top}\left(\tilde{\mathcal{I}}_{t \mid t}^{x}\right)^{-1} \geq 0$, and by using (3.25) in the second equality. Since the inequality also holds for all square submatrices along the diagonal, it 
follows that $\tilde{P}_{t \mid t}^{p} \geq P_{t \mid t}^{p}$, where $\tilde{P}_{t \mid t}^{p}$ and $P_{t \mid t}^{p}$ are the submatrices of $\tilde{P}_{t \mid t}^{x}$ and $P_{t \mid t}$ related to the platform position and orientation estimates. Note that, in the limit, as $M \rightarrow \infty$, (3.32) turns into a Riemann integral and (3.9) is attained. In conclusion, the introduced approximation in the information-bound approach, where infinitely many unknown landmarks are represented with a single subregion, i.e., (3.9), thereby underestimates the true covariance of the platform position and orientation estimates, and conversely overestimates the true information of the estimates.

\subsection{Numerical evaluation}

In this section, the presented approaches are evaluated. The platform is assumed to obtain measurements of nearby landmarks using an onboard range-bearing sensor, e.g., a LiDAR, with a limited range of $100 \mathrm{~m}$, as described in Section 2.3.2. In the evaluation, the measurement noise $\epsilon_{t}^{i}$ is

$$
\epsilon_{t}^{i} \sim \mathcal{N}\left(0,\left[\begin{array}{cc}
0.2^{2} & 0 \\
0 & (\pi / 180)^{2}
\end{array}\right]\right),
$$

The upper bound of the gain in the information matrix from observations in the information-bound approach, i.e.,

$$
\overline{\mathcal{I}}^{i}=\sum_{k=1}^{K} \overline{\mathcal{I}}^{i, k} \rho_{i}^{\max },
$$

is evaluated for $K \in\{10,15,20,40\}$ and compared to the point approximation in (3.11), here denoted $\mathcal{I}_{1 \times 1}^{P A}$. The information matrix is calculated for a single subregion $\Omega_{i}$ of size $40 \times 40 \mathrm{~m}^{2}$, with $\rho(m)=1$ for all $m \in \Omega_{i}$. The quality of the different approximations are calculated for a sensor position between 21 and 60 meters away from the center of $\Omega_{i}$, and all sensor positions are evaluated independently. The calculated information matrix is then compared with the integrated information matrix $\mathcal{I}_{t}^{i}$ in (3.9), computed with numerical integration in Wolfram Mathematica (Wolfram Research, Inc., 2019). The differences between these matrices are then evaluated with the Frobenius norm as

$$
\left\|\left(\mathcal{I}_{t}^{i}\right)^{-1 / 2}\left(\mathcal{I}_{t}^{i}-\mathcal{I}^{\bullet}\right)\left(\mathcal{I}_{t}^{i}\right)^{-1 / 2}\right\|_{F},
$$

with $\overline{\mathcal{I}}^{i}$ and $\mathcal{I}_{1 \times 1}^{P A}$ substituting $\mathcal{I} \bullet$. The difference is normalized by multiplying with $\left(\mathcal{I}_{t}^{i}\right)^{-1 / 2}$ from the left and right to account for the different units in $\mathcal{I}_{t}^{i}$.

Note that, errors in the information gained using the point-approximation approach and the information-bound approach arise from different factors, i.e., from the range and the bearing measurements respectively. By increasing or decreasing the corresponding elements in the $R$ matrix, the relation of the difference $\mathcal{I}_{t}^{i}-\mathcal{I}_{1 \times 1}^{P A}$ and $\mathcal{I}_{t}^{i}-\overline{\mathcal{I}}^{i}$ changes. This makes the use of the criterion in (3.15) problematic when comparing these approaches. Instead, (3.39) is used as it is found to be less susceptible to this effect. 


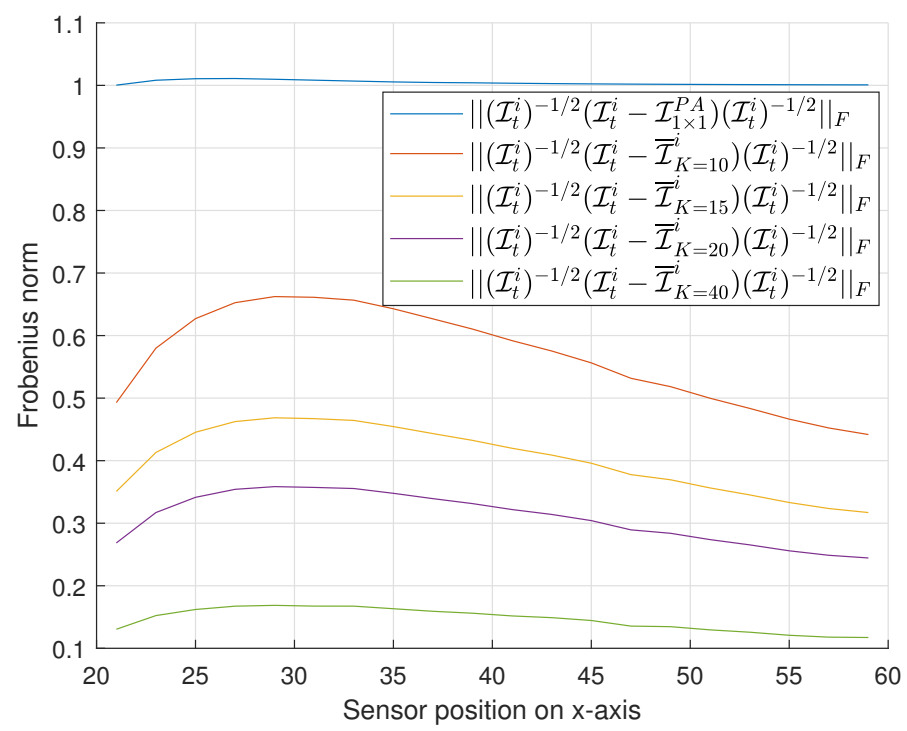

Figure 3.7: Frobenius norm of the difference between $\mathcal{I}_{t}^{i}$ and the information matrix estimated with a point approximation $\mathcal{I}_{1 \times 1}^{P A}$ as well as $\overline{\mathcal{I}}^{i}$ with different number of sectors $K$. The normalizing term $\left(\mathcal{I}_{t}^{i}\right)^{-1 / 2}$ is used to account for the different units in $\mathcal{I}_{t}^{i}$.

As seen in Figure 3.7, the information-bound approach has a smaller error for all distances compared to the point approximation. Furthermore, for larger $K$ the error decreases significantly. Tests indicate that the error can be made arbitrarily small by iteratively increasing $K$. The increase in error, peaking at $x=30$, for the information-bound approach, is due to the misalignment of the geometries of the circle sectors and the square subregion. At this peak, some of the sectors are almost at a 45 degree angle to the subregion, thus a larger portion of the sectors are outside of the region, similar to what can be seen in Figure 3.5. 



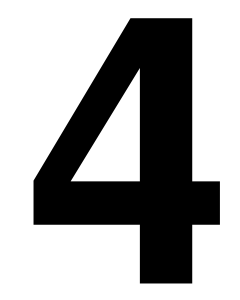

\section{Landmark detection}

A sensor can generally not detect all landmarks within its sensor's range due to, e.g., low target visibility or occlusion by other landmarks. Therefore, including the probability of landmark detection during planning is important as the information gained from observing a region would otherwise be overestimated.

In this chapter, an approach for computing the landmark-detection probabilities in ground-to-ground LiDAR-data is presented. This approach is evaluated in realistic scenarios where a real landmark detector is used for detection in a simulated point cloud. More specifically, pole-based landmarks, such as the stems of pine trees, are to be detected. These detection probabilities are then used to scale the estimated information gained from observed subregions during the planning phase in later chapters. This analysis is based upon the landmark detector found in Section 2.4. The modeled factors affecting the landmark-detection probability are divided into two main sources. These are

- the probability that the ray path from the sensor to the landmark is free; and

- the probability that a landmark in subregion $\Omega_{i}$ is large enough to be detected at that range.

These probabilities are denoted $p^{\text {free }}$ and $p_{\Omega_{i}}^{\text {size }}$ respectively. Note that other sources affecting the ability to detect landmarks exist, such as incorrect association, attenuation in the atmosphere and low target reflectivity (Wojtanowski et al., 2014). However, these are outside of the scope of this work.

The computations involved in determining $p^{\text {free }}$ are described in Section 4.1 and Section 4.2. When using LiDAR-measurements to detect the tree landmarks, such as when using the pole-landmark detector described in Section 2.4, the diameters of the trees have a large influence on whether the tree is detected or 
not. The distribution of the tree diameters is thereby a highly informative metric that should be included when predicting the future information gained from observing a region. An approach for estimating the tree-diameter distribution in a subregion $\Omega_{i}$ is therefore presented in Section 4.3. These distributions are used in Section 4.4 to compute $p_{\Omega_{i}}^{\text {size }}$. Section 4.5 describes how these probabilities can be integrated in the previously presented information estimation process using virtual landmarks. A numerical evaluation of the proposed approach is then presented in Section 4.6.

\subsection{Ray-occlusion probability}

Similar to (Schaefer et al., 2017), it is assumed that the prior probability that a ray travels through a specific volume unobstructed follows an exponential decay process. For such a process, the probability that a ray travels a distance greater than or equal to $r$ from the sensor position $p_{t}$ to the landmark position $m$ is given by

$$
P\left(r \mid \zeta, p_{t}, m\right)=\exp \left(-\int_{\tau=0}^{r} \zeta\left(\tau, p_{t}, m\right) d \tau\right),
$$

where $\zeta(\cdot)$ is the decay rate along the ray path. However, in this analysis, it is assumed that the decay rate within each subregion $\Omega_{i}$, introduced in Chapter 3 , is constant and given by tree stem ground surface coverage, i.e., the basal area $A_{i}^{\mathrm{BA}}$ defined in Section 3.1. That is, if a large percentage of the ground area is covered by cylinder-shaped trees then any ray traveling through the volume has lower probability of passing through the subregion unobstructed. With this assumption, (4.1) can be simplified.

For a ray traveling from the sensor position to a landmark at a distance $r$ from the sensor, the ray is first divided into ray segments $r_{i}$, where each segment $r_{i}$ represents the length of the ray-path through the subregion $\Omega_{i}$ as seen in Figure 4.1. The probability of the ray traveling the entire length $r=\sum_{i=1}^{\mathcal{S}} r_{i}$ from the sensor through the subregions $\Omega_{1}, \Omega_{2}, \ldots, \Omega_{\mathcal{S}}$ is given by

$$
P\left(r_{1}, r_{2}, \ldots, r_{\mathcal{S}} \mid \rho_{1}^{\mathrm{BA}}, \rho_{2}^{\mathrm{BA}}, \ldots, \rho_{\mathcal{S}}^{\mathrm{BA}}\right)=\exp \left(-\sum_{i=1}^{\mathcal{S}} A_{i}^{\mathrm{BA}} r_{i}\right)
$$

Note that computing the ray-segments $r_{i}$ can be done efficiently by using, e.g., the VisitCellsOverlapped-algorithm in (Ericson, 2004).

\subsection{Derivation of $p^{\text {free }}$}

In order to calculate the probability that enough LiDAR-rays reach the landmark, a few approximations are made. First, for ease of computation, it is assumed that the probability of a ray hitting the landmark is equal to the probability of a ray making it to the center point of the landmark. That is, the geometry of 


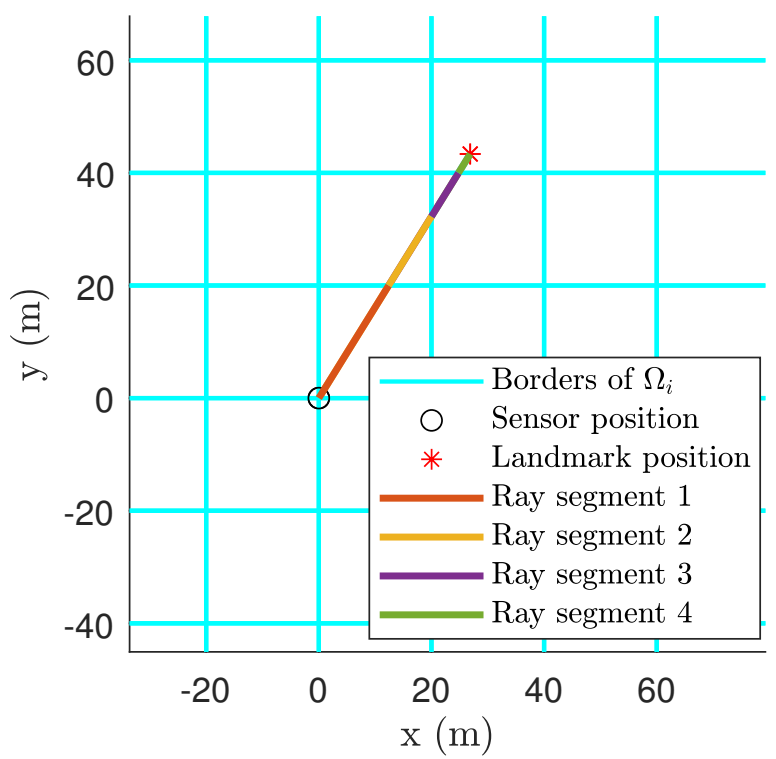

Figure 4.1: Illustration of the partitioning of a ray, traveling from the sensor position to a landmark, into segments $r_{i}, i \in\{1,2,3,4\}$.

the landmarks themselves and the small angular difference between points hitting the landmark are not considered. Given that the diameter of the considered landmarks is several orders of magnitude smaller than the range to the sensor, this simplification has a negligible effect on the overall accuracy of the model. As a second approximation, it is assumed that the probability of rays reaching a landmark is independent of each other. This is not necessarily true. If one ray reaches the landmark unobstructed, it is likely that any ray next to it will also do so. However, no model to determine this dependence based on the available prior information is known, hence this approximation is used.

Recall the pole-landmark detector in Section 2.4. For a landmark to be detected, two requirements need to be fulfilled. From the second requirement it follows that no object can be in front of the segments covering the landmark on the four rows closest to the horizon plane. Furthermore, the points in the columns immediately on the left and right side of these segments need to be behind the landmark in order to determine the edge of the landmark, as a point in front of the landmark in these columns cannot be distinguished from an obstacle. An illustration of this can be seen in Figure 4.2. Assume each segment is intersected by $n$ LiDAR-rays. This means that $n+2$ rays on each of the four rows, a total of $4(n+2)$ rays, need to reach a distance greater than or equal to the distance to the landmark.

Given the above approximations the prior probability that all of the required 


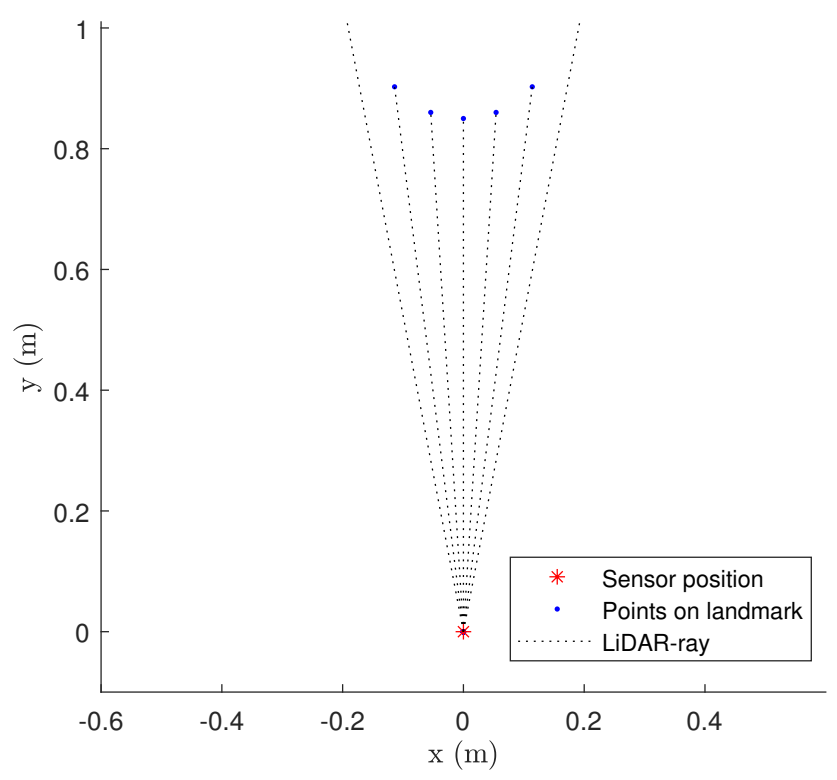

Figure 4.2: Illustration of LiDAR-rays (dotted black lines) traveling from the sensor position (red) to a single segment consisting of $n=5$ points (blue). The rays on the left and right side of the segment need to be behind the landmark in order to determine its edges. If any of these rays would be obstructed by an object in front of the landmark, the landmark would not be detected. 
rays reach the landmark at position $m$ from the sensor position $p_{t}$, is given by

$$
p^{\text {free }}\left(n, p_{t}, m\right)=P\left(r_{1}, r_{2}, \ldots, r_{\mathcal{S}} \mid \rho_{1}^{\mathrm{BA}}, \rho_{2}^{\mathrm{BA}}, \ldots, \rho_{\mathcal{S}}^{\mathrm{BA}}\right)^{4(n+2)} \text {. }
$$

\subsection{Estimating tree-diameter distribution}

In order to determine the probability that a pole-based landmark is detected, the distribution of the landmark diameter in a subregion $\Omega_{i}$ needs to be known. The following describes how this distribution can be extracted from prior data.

The tree stem diameter is modeled using a two-parameter Weibull distribution. Since the shape and scale parameters of this distribution are not easily computed using prior knowledge, a parameter recovery method is applied. This approach uses a Newton-Raphson method (Ben-Israel, 1966) for root-finding of a system of two nonlinear equations derived from the Weibull-distribution. In the following, a derivation of the parameter recovery method based on (Siipilehto and Mehtätalo, 2013) is presented.

Let the random variable $D$ denote the diameter of a tree in subregion $\Omega_{i}$ and let it follow a Weibull-distribution with the scale parameter $\lambda_{\Omega_{i}}$ and shape parameter $\kappa_{\Omega_{i}}$. The parameters $\lambda_{\Omega_{i}}$ and $\kappa_{\Omega_{i}}$ are recovered by matching the expected value of the quadratic mean diameter $\bar{d}_{i}^{\mathrm{QM}}$ and the basal-area weighted mean diameter $\bar{d}_{i}^{\mathrm{BA}}$, for a subregion $\Omega_{i}$, with their empirical counterpart. The basalarea weighted mean diameter is given in the national forest data presented in Section 3.1.1, but the quadratic mean diameter is not. However, the quadratic mean diameter can be approximated using the basal area $A_{i}^{\mathrm{BA}}$ and the estimated number of trees $\tilde{N}_{i}$ defined in (3.2). The basal area is defined as

$$
A_{i}^{\mathrm{BA}}=\sum_{j=1}^{N_{i}} \pi\left(\frac{d_{j}}{2}\right)^{2},
$$

where $d_{j}$ is the diameter of the $j$ th tree in the subregion and $N_{i}$ is the number of trees in $\Omega_{i}$. The quadratic mean diameter of $\Omega_{i}$, defined as

$$
\bar{d}_{i}^{\mathrm{QM}}=\frac{1}{N_{i}} \sum_{j=1}^{N_{i}} d_{j}^{2},
$$

can be approximated using (4.4) as

$$
\bar{d}_{i}^{\mathrm{QM}}=\frac{1}{N_{i}} \sum_{j=1}^{N_{i}} d_{j}^{2} \approx \frac{4 A_{i}^{\mathrm{BA}}}{\pi \tilde{N}_{i}},
$$

where the approximation follows from the use of $N_{i} \approx \tilde{N}_{i}$.

Next, analytical expressions for the expected value of the quadratic mean diameter and the basal-area weighted mean diameter will be determined. Note 
that, as shown in (Weibull, 1951), the $j$ th moment of the two-parameter Weibull distribution is

$$
E\left(D^{j}\right)=\lambda_{\Omega_{i}}^{j} \Gamma\left(j / \kappa_{\Omega_{i}}+1\right),
$$

where $\Gamma(\cdot)$ is the gamma function (Olver et al., 2010) defined as

$$
\Gamma(z)=\int_{0}^{\infty} e^{-t} t^{z-1} d t
$$

for any $z$ with strictly positive real part. The expected quadratic mean diameter is then given by the second moment, i.e.,

$$
E\left(\bar{d}_{i}^{\mathrm{QM}}\right)=E\left(D^{2}\right)=\lambda_{\Omega_{i}}^{2} \Gamma\left(2 / \kappa_{\Omega_{i}}+1\right) .
$$

Similarly, as shown in (Siipilehto and Mehtätalo, 2013), the expected basal-area weighted mean diameter is given by

$$
E\left(\bar{d}_{i}^{\mathrm{BA}}\right)=\frac{E\left(D^{3}\right)}{E\left(D^{2}\right)}=\lambda_{\Omega_{i}} \frac{\Gamma\left(3 / \kappa_{\Omega_{i}}+1\right)}{\Gamma\left(2 / \kappa_{\Omega_{i}}+1\right)} .
$$

Using the Newton-Raphson method, the scale and shape parameters $\lambda_{\Omega_{i}}$ and $\kappa_{\Omega_{i}}$ can then be recovered by finding the roots to the system defined by the equations

$$
\begin{aligned}
\lambda_{\Omega_{i}} \frac{\Gamma\left(3 / \kappa_{\Omega_{i}}+1\right)}{\Gamma\left(2 / \kappa_{\Omega_{i}}+1\right)}-\bar{d}_{i}^{\mathrm{BA}} & =0 \\
\lambda_{\Omega_{i}}^{2} \Gamma\left(2 / \kappa_{\Omega_{i}}+1\right)-\frac{4 A_{i}^{\mathrm{BA}}}{\pi \tilde{N}_{i}} & =0 .
\end{aligned}
$$

\subsection{Derivation of $p_{\Omega_{i}}^{\text {size }}$}

Since the rays from the considered LiDAR-model disperse radially from the sensor center, the distance between two points on neighboring rays increases with the distance from the sensor. This makes detection of a landmark at a long distance harder than at a short distance, since the point cloud becomes less dense and gives the sensor a lower effective detection range. The diameters of landmarks thereby need to be larger at longer distances from the sensor in order to be detected. In the following, the probability that a landmark is large enough to be detected at a certain distance is derived as a function of the distribution of its diameter.

The minimal number of possible points $n$ on a pole-based landmark with diameter $D$ at distance $r$ is given by

$$
n=2 \tan ^{-1}\left(\frac{D}{2 r}\right) \frac{N_{W}}{\alpha_{F O V}}=\phi \frac{N_{W}}{\alpha_{F O V}},
$$




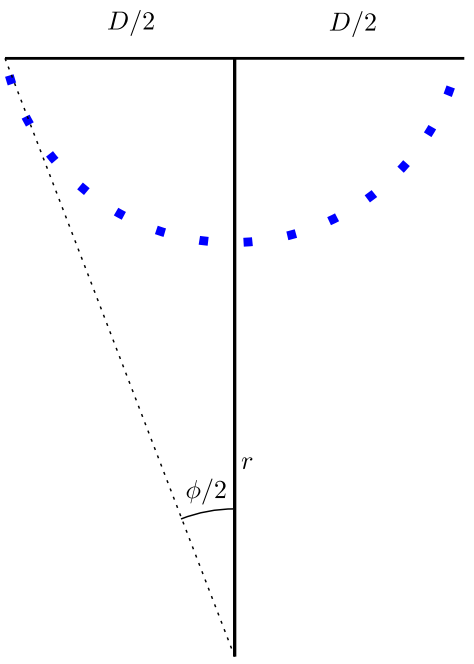

Figure 4.3: Illustration of the relationship between the landmark diameter $D$, the range $r$ from the sensor and the angle $\phi$ used to determine the number of points in a point cloud that will cover the landmark.

where $N_{W}$ is the number of points on each row of the point cloud, $\phi=2 \tan ^{-1}\left(\frac{D}{2 r}\right)$ is the angle between the outermost points of the pole-based landmark from the sensor position, and $\alpha_{F O V}$ is the angular field of view of the sensor in radians. The relationship of these variables can be seen in Figure 4.3. Note, while (4.12) describes the minimal number of points on a landmark with diameter $D$, it is possible to get more if, e.g., one of the LiDAR-rays is aligned with the edge of the landmark. However, since the alignment of LiDAR-rays is unknown a priori, (4.12) is used as an approximation.

Using (4.12), the probability that a landmark in subregion $\Omega_{i}$ has a diameter $D$ that is large enough to include $n=j$ points at range $r$, from the sensor, is given by

$$
\begin{aligned}
p_{\Omega_{i}}^{\text {size }} & (n=j)=P\left(n=j \mid \Omega_{i}\right)=P\left(n<j+1 \mid \Omega_{i}\right)-P\left(n<j \mid \Omega_{i}\right) \\
& =P\left(2 \tan ^{-1}\left(\frac{D}{2 r}\right) \frac{N_{W}}{\alpha_{F O V}}<j+1 \mid \Omega_{i}\right)-P\left(2 \tan ^{-1}\left(\frac{D}{2 r}\right) \frac{N_{W}}{\alpha_{F O V}}<j \mid \Omega_{i}\right) \\
& =P\left(\frac{D}{2 r}<\tan \left(\frac{(j+1) \alpha_{F O V}}{2 N_{W}}\right) \mid \Omega_{i}\right)-P\left(\frac{D}{2 r}<\tan \left(\frac{j \alpha_{F O V}}{2 N_{W}}\right) \mid \Omega_{i}\right) \\
& =P\left(D<2 r \tan \left(\frac{(j+1) \alpha_{F O V}}{2 N_{W}}\right) \mid \Omega_{i}\right)-P\left(D<2 r \tan \left(\frac{j \alpha_{F O V}}{2 N_{W}}\right) \mid \Omega_{i}\right) \\
& =\mathcal{F}\left(2 r \tan \left(\frac{(j+1) \alpha_{F O V}}{2 N_{W}}\right) ; \kappa_{\Omega_{i}}, \lambda_{\Omega_{i}}\right)-\mathcal{F}\left(2 r \tan \left(\frac{j \alpha_{F O V}}{2 N_{W}}\right) ; \kappa_{\Omega_{i}}, \lambda_{\Omega_{i}}\right),
\end{aligned}
$$


where $\mathcal{F}\left(\cdot ; \kappa_{\Omega_{i}}, \lambda_{\Omega_{i}}\right)$ denotes the cumulative distribution function of the Weibull distribution, with shape and scale parameters $\kappa_{\Omega_{i}}$ and $\lambda_{\Omega_{i}}$, describing the distribution of the tree stem diameters in subregion $\Omega_{i}$, as defined in Section 4.3.

The probability of detecting a landmark covered by $n$ points, at a position $m \in \Omega_{i}$ from the sensor position $p_{t}$, can then be computed by multiplying (4.3) and (4.13), yielding

$$
p^{\text {detect }}\left(n, p_{t}, m\right)=p_{\Omega_{i}}^{\text {size }}(n) p^{\text {free }}\left(n, p_{t}, m\right)
$$

The marginal probability of detecting a landmark of any size at position $m \in \Omega_{i}$ from the sensor position $p_{t}$ can be obtained by summing the joint probability in (4.14) over $n$, yielding

$$
p^{\text {detect }}\left(p_{t}, m\right)=\sum_{n=3}^{\infty} p_{\Omega_{i}}^{\text {size }}(n) p^{\text {free }}\left(n, p_{t}, m\right) \text {. }
$$

Note that the summation starts at $n=3$ since the requirement that the merged segments include at least nine points on the four rows closest to the horizontal plane, as presented in Section 2.4, cannot otherwise be fulfilled.

\subsection{Information estimation}

For a platform equipped with a LiDAR-sensor and using the modeled landmark detector, the expected information gained from observing a subregion $\Omega_{i}$ should be computed as

$$
\mathcal{I}_{t}^{i}=\int_{\tilde{m} \in \Omega_{i}} p^{\operatorname{detect}}\left(p_{t}, \tilde{m}\right) \rho(\tilde{m}) \mathcal{I}\left(p_{t}, \tilde{m}\right) d \tilde{m} .
$$

However, computing $p^{\text {detect }}(\cdot)$ for a large number of points is computationally demanding since it relies on a ray-casting approach to compute (4.2). Instead, using the information-bound approach introduced in Section 3.3 in conjunction with the above probabilities, the approximate upper and lower bounds $\underline{\mathcal{I}}_{t}^{i}$ and $\overline{\mathcal{I}}_{t}^{i}$ on $\mathcal{I}_{t}^{i}$ can be computed as

$$
\begin{aligned}
& \underline{\underline{I}}_{t}^{i}=\sum_{k=1}^{K} \underline{\mathcal{I}}_{t}^{i, k} \rho_{i}^{\min } p^{\operatorname{detect}}\left(p_{t}, \bar{m}^{i}\right) \\
& \overline{\mathcal{I}}_{t}^{i}=\sum_{k=1}^{K} \overline{\mathcal{I}}_{t}^{i, k} \rho_{i}^{\max } p^{\operatorname{detect}}\left(p_{t}, \bar{m}^{i}\right)
\end{aligned}
$$

where $\rho_{i}^{\max }$ and $\rho_{i}^{\min }$ is the maximum and minimum density, respectively, in a subregion $\Omega_{i}$ and $\bar{m}^{i}$ is the center point in $\Omega_{i}$. 


\subsection{Numerical evaluation}

The following sections include a numerical evaluation of the presented approach. The evaluation is divided into two parts. In the first part, the accuracy of the landmark-detection probability is evaluated by comparing the estimated number of detectable landmarks with the actual number of detected landmarks. In the second part, the effects of the probabilities $p^{\text {free }}(\cdot)$ and $p_{\Omega_{j}}^{\text {size }}(\cdot)$ on the detection probability $p^{\text {detect }}(\cdot)$ is investigated. Since the evaluation is based upon a real landmark detector, a simulation environment is first presented. This simulation environment is used to generate point clouds which are used as input to the detector.

\subsubsection{Simulation environment}

The simulation environment is designed to generate point clouds representative of those from an Ouster LiDAR OS1-16 (Ouster, Inc., 2021). This sensor has a field of view of $\alpha_{F O V}=2 \pi$ and generates a complete point cloud with 16 rows and $N_{W}=2048$ columns at $5 \mathrm{~Hz}$. The horizontal rows are spread evenly with elevations between $+15^{\circ}$ and $-15^{\circ}$, with the center line at $0^{\circ}$ elevation. It is assumed that the sensor is mounted on a platform at a height of $2 \mathrm{~m}$ above the ground plane. The ground plane is modeled by a flat surface.

Given the size and position of landmarks, a point cloud is generated by computing where the LiDAR-rays intersect with the objects in the environment, e.g., the landmarks. For pole-based landmarks, this can be done by using the IntersectSegmentCylinder-algorithm in (Ericson, 2004). An example of a generated point cloud can be seen in Figure 4.4 .

In order to generate an accurate point cloud, realistic landmark positions are needed. Using tree stems as landmarks puts a constraint on how close landmarks can be to one another. Since it is unknown how these positions should be randomly generated, correct landmark positions are instead generated from detected trees in orthophotos of the area using a visual tree detector, as described in Section 3.1.2. The stem diameter of these trees are then sampled from the underlying Weibull-distribution, defined in Section 4.3. Note that, in order to avoid bias, only areas where the number of trees detected using the visual tree detector match the densities generated using the method in Section 3.1.1 are considered.

\subsubsection{Estimated number of landmark detections}

The accuracy of the landmark-detection probability is evaluated in this section. This is done by comparing the expected number of landmark detections with the actual number of detected landmarks for 100 random regions, using a simulated point cloud and a real landmark detector.

For each region $j \in\{1,2, \cdots, 100\}$, the expected number of detectable trees, 


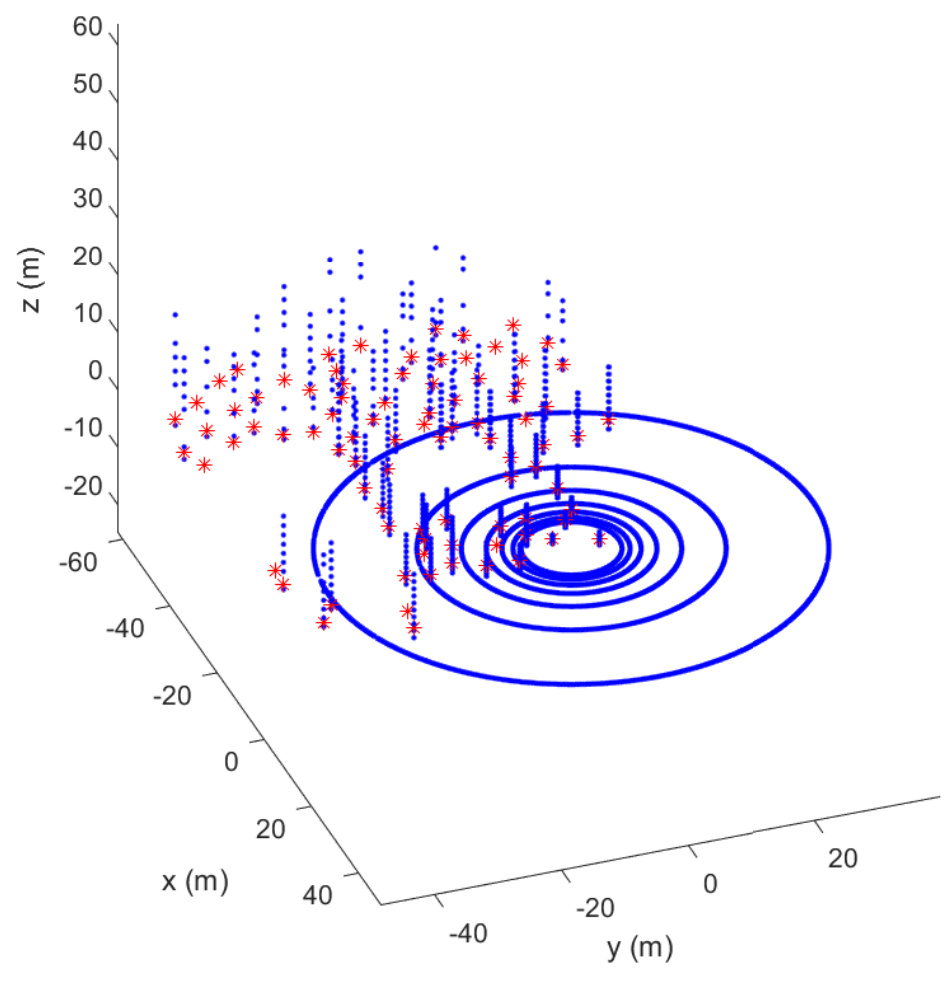

Figure 4.4: Illustration of a generated point cloud using the proposed simulation environment. The points in the point cloud are marked in blue while the center position of the landmarks are marked in red. The blue rings are formed by LiDAR-rays intersecting the ground surface. Notice that landmarks far from the sensor origin are covered by fewer points due to the point cloud becoming less dense. 
denoted $N_{\exp }^{j}$, is calculated as

$$
N_{\exp }^{j}=\sum_{i=1}^{M} p^{\operatorname{detect}}\left(\bar{p}_{t}, \bar{m}^{i}\right) \rho_{i}\left|\Omega_{i}\right|,
$$

where $\left|\Omega_{i}\right|$ denotes the area of the subregion $\Omega_{i}, M$ is the number of subregions in the region, and $\bar{m}^{i}$ is the subregion midpoint. Thus, the detection probability is calculated for a ray traveling from the region midpoint $\bar{p}_{t}$ to each subregion midpoint $\bar{m}^{i}$. The size of each region is $200 \times 200 \mathrm{~m}^{2}$ and is divided into subregions of size $12.5 \times 12.5 \mathrm{~m}^{2}$. These regions are randomly selected in and around forested areas in the northern part of Sweden.

The process of determining the number of real detections, denoted $N_{\text {detect }}^{j}$, in a region $j$ consists of several steps. Tree positions are first determined by applying the visual tree detector in Section 3.1.2 to the orthophotos corresponding to each region. These tree positions are then used to generate a simulated point cloud, as described in Section 4.6.1. The pole-landmark detector, described in Section 2.4, is then applied to the generated point cloud, yielding the landmark detections used in the evaluation.

For the landmark-detection probabilities to be accurate, the sample mean

$$
\bar{z}=\frac{1}{100} \sum_{j=1}^{100} z^{j}
$$

should be unbiased, where $z^{j}=N_{\text {detect }}^{j}-N_{\text {exp }}^{j}$. This is tested using a one-sample t-test (Montgomery, 2009). In this test it is assumed that the samples $z^{j}$ are independent identical distributed from $\mathcal{N}\left(\mu_{0}, \sigma^{2}\right)$, where $\mu_{0}$ and $\sigma$ are unknown parameters. The test statistic used in the t-test is defined as

$$
t=\frac{\bar{z}-\mu_{0}}{s / \sqrt{n}}
$$

where $s$ is the sample standard deviation. The null hypothesis is that $\mu_{0}$ is zero while the alternative hypothesis is that it is not, i.e.:

$$
\begin{aligned}
& H_{0}: \mu_{0}=0 \\
& H_{1}: \mu_{0} \neq 0
\end{aligned}
$$

The 95\%-confidence interval of the test statistic is $(-0.8429,3.6668)$. As this confidence interval includes 0 , the null hypothesis cannot be rejected.

Since each region is randomly selected from different parts of northern Sweden, the independence assumption of $z^{j}$ is considered fulfilled. The normality assumption of $z^{j}$ is tested using a Lilliefors test (Lilliefors, 1967). This test showed that the null hypothesis, that the statistic comes from a normal distribution, could not be rejected with $95 \%$ confidence. The associated normality plot and a histogram of the statistic can be seen in Figure 4.5. Although these plots show some signs of heavy tails, these are not considered enough to reject the normality assumption. 

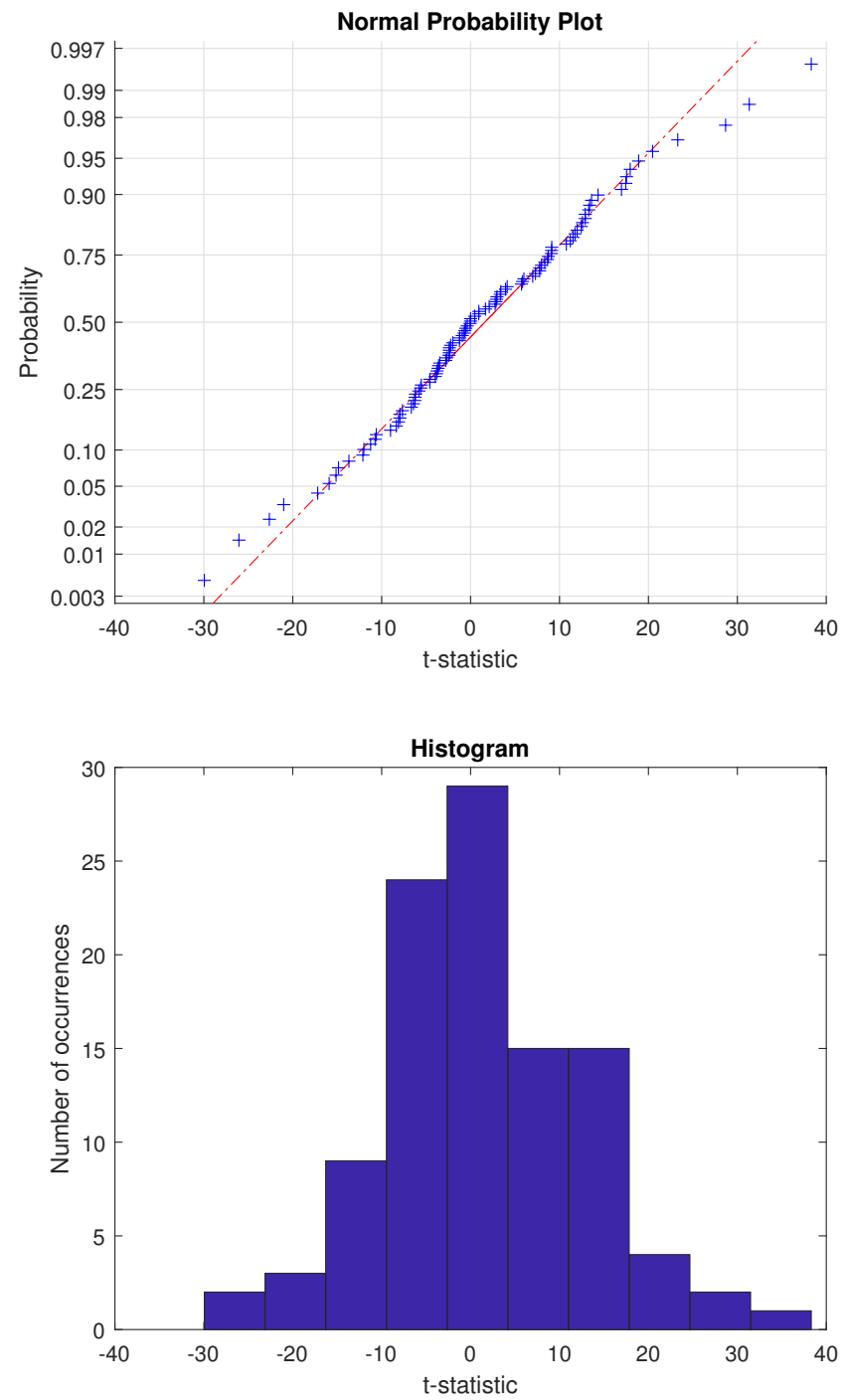

Figure 4.5: Distribution of the t-statistic used to test the estimated number of landmarks against the actual number of detected landmarks. Upper: The $t$-statistic fitted onto a normality probability plot, where it follows the center line well. Lower: Histogram of the t-statistic. To a degree, the t-statistic shows some heavy tails but not enough to violate the normality assumption used in the test. 


\subsubsection{Effects of the probabilities $p^{\text {free }}$ and $p_{\Omega_{j}}^{\text {size }}$}

In this section, the effects of $p^{\text {free }}(\cdot)$ and $p_{\Omega_{j}}^{\text {size }}(\cdot)$ on the detection probability $p^{\text {detect }}(\cdot)$ are investigated. Two sensors, denoted $p_{1}$ and $p_{2}$, are placed at different ranges from a single landmark at a position $m$. The $p_{1}$ sensor is placed in an area with many trees while the $p_{2}$ sensor is placed in an open field, as seen in Figure 4.6. The probability that the ray paths are free of obstacles, i.e., $p^{\text {free }}(\cdot)$ in $(4.3)$, and the probability that the landmark is large enough to include $n=3$ and $n=5$ points, i.e., $p_{\Omega_{i}}^{\text {size }}(\cdot)$ in $(4.13)$, are then calculated for all sensor positions. These can be seen in Table 4.1 and Table 4.2 .

As seen in these tables, in relative terms, the probability $p_{\Omega_{i}}^{\text {size }}(\cdot)$ decreases faster than $p^{\text {free }}(\cdot)$, for both increasing $r$ and increasing $n$. Thus, $p_{\Omega_{i}}^{\text {size }}(\cdot)$ will be the dominating factor in $p^{\text {detect }}(\cdot)$. If the platform is close to or far from a landmark thereby has a much larger impact on the landmark-detection probability than if the platform is in an open or occluded environment. Note that, since the subregion closest to the landmark is not free of obstacles, the probability $p^{\text {free }}(\cdot)$ is less than one, but constant, for all sensor positions of $p_{2}$.

Table 4.1: The table shows the probability $p_{\Omega_{i}}^{\text {size }}(n)$, of the landmark being large enough to be covered by $n=3$ points, and the probability $p^{\text {free }}\left(n, p_{1}, m\right)$ and $p^{\text {free }}\left(n, p_{2}, m\right)$ that enough rays are reaching the landmark in order for it to be detected by sensor $p_{1}$ and $p_{2}$ respectively.

\begin{tabular}{|c|c|c|c|}
\hline$r$ & $p_{\Omega_{i}}^{\text {size }}(n)$ & $p^{\text {free }}\left(n, p_{1}, m\right)$ & $p^{\text {free }}\left(n, p_{2}, m\right)$ \\
\hline 30 & 0.094 & 0.422 & 0.673 \\
40 & 0.038 & 0.319 & 0.673 \\
50 & 0.012 & 0.187 & 0.673 \\
60 & 0.003 & 0.110 & 0.673 \\
70 & 0.001 & 0.067 & 0.673 \\
\hline
\end{tabular}

Table 4.2: The table shows the probability $p_{\Omega_{i}}^{\text {size }}(n)$, that the landmark being large enough to be covered by $n=5$, points and the probability $p^{\text {free }}\left(n, p_{1}, m\right)$ and $p^{\text {free }}\left(n, p_{2}, m\right)$ that enough rays are reaching the landmark in order for it to be detected by sensor $p_{1}$ and $p_{2}$ respectively.

\begin{tabular}{|c|c|c|c|}
\hline$r$ & $p_{\Omega_{i}}^{\text {size }}(n)$ & $p^{\text {free }}\left(n, p_{1}, m\right)$ & $p^{\text {free }}\left(n, p_{2}, m\right)$ \\
\hline 30 & 0.010 & 0.299 & 0.574 \\
40 & 0.001 & 0.202 & 0.574 \\
50 & 0.000 & 0.096 & 0.574 \\
60 & 0.000 & 0.046 & 0.574 \\
70 & 0.000 & 0.023 & 0.574 \\
\hline
\end{tabular}




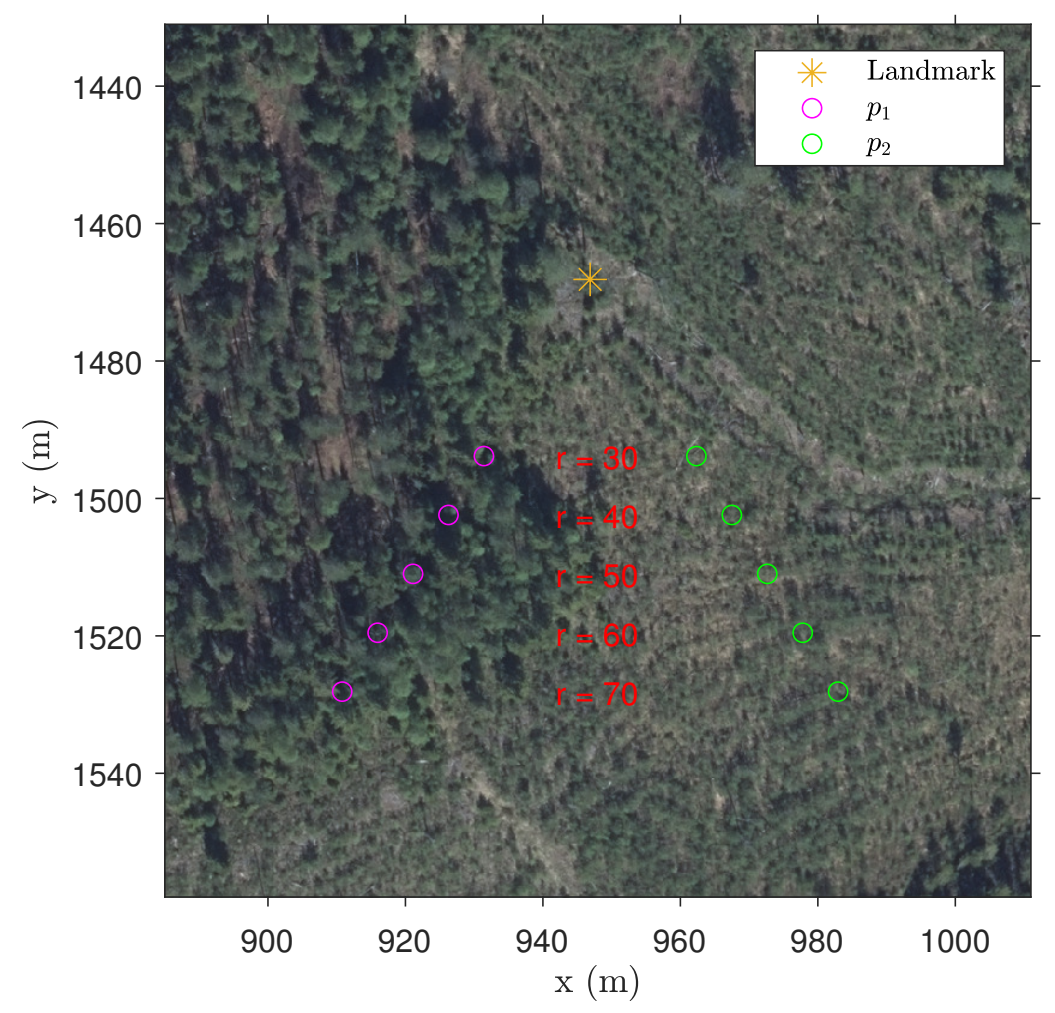

Figure 4.6: Illustration of an evaluation scenario where two sensors, denoted $p_{1}$ (magenta) and $p_{2}$ (green), are observing a landmark (orange) at different ranges. Sensor $p_{1}$ observes the landmark through a forest while $p_{2}$ observes the landmark through an open field. 


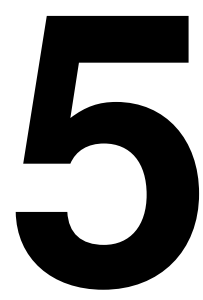

\section{Motion planning for SLAM}

This chapter introduces a planning approach which aims to go beyond the current limitations of having complete prior knowledge of the environment in beliefspace planning. This is achieved by using landmark densities represented by virtual landmarks, rather than the explicit landmarks. The introduction of virtual landmarks removes the requirement for prior knowledge of landmarks positions, which is the case in some belief-space planning algorithms.

This chapter begins with a brief summary of the studied belief-space planning problem. Then, it is shown how to find a solution to this problem by using the introduced virtual landmarks and the estimated landmark-detection probabilities. Finally, a numerical evaluation of the proposed approach is presented.

\subsection{Planning with unknown landmarks}

Recall the formulation of the belief-space planning problem in (2.46). In this formulation, the process and measurement noise realizations are exchanged with their expected value. Furthermore, the state distribution is approximated using the EIF-SLAM algorithm. This mitigates the problem of the unknown noise realizations at planning time.

The problem of unknown landmarks still remains. A common method in previous belief-space planning approaches is to only consider the already detected landmarks during the planning phase, thereby disregarding the possibility of detecting new landmarks. This leads to an underestimation of the information gained from observing unexplored regions of the environment. By introducing landmark densities as environment priors and representing these with virtual landmarks, as described in Chapter 3, the information gained from possible, but unknown, landmarks can be included in the planning phase. In the presented problem, no knowledge of explicit landmarks is assumed to be available. The 
measurement update is thereby performed with virtual landmarks, rather than real landmarks, during planning. In the spirit of certainty-equivalent control, these virtual landmarks are constructed in order to capture the expected information gained from observing the surrounding subregion. Note that, a benefit of the current problem formulation is that virtual landmarks can be used in conjunction with real landmarks. Hence, any known landmarks can be included in a straight-forward manner.

The considered belief-space planning problem can then be solved by finding the parameters $\left\{\pi_{t}\right\}_{t=1}^{T}$ of a policy $\pi$ that solves the optimization problem

$$
\begin{aligned}
\underset{\left\{\pi_{t}\right\}_{t=1}^{T}, T}{\operatorname{minimize}} & J\left(\hat{x}_{0: T}, \mathcal{I}_{0: T}\right) \\
\text { subject to } & \hat{x}_{t \mid t}=f\left(\hat{x}_{t-1 \mid t-1}, u_{t-1}, 0\right), \\
& \hat{y}_{t}^{i}=h^{i}\left(\hat{x}_{t \mid t}\right), \quad \forall i \in \mathcal{M}_{t}, \\
& \mathcal{I}_{t \mid t}=\Lambda\left(\mathcal{I}_{t-1 \mid t-1}, \hat{y}_{t}, \hat{x}_{t-1 \mid t-1}, u_{t-1}\right) \\
& u_{t}=\pi\left(\hat{x}_{t \mid t}, \mathcal{I}_{t \mid t}, \pi_{t+1}\right) \\
& \hat{x}_{0 \mid 0}=x_{i n i t}, \\
& \hat{x}_{T \mid T} \in \mathcal{X}_{\text {goal }}, \\
& \hat{x}_{t \mid t} \in \mathcal{X}, \\
& u_{t} \in \mathcal{U}\left(\hat{x}_{t \mid t}\right),
\end{aligned}
$$

where $\hat{y}_{t}=\left\{\hat{y}_{t}^{i}\right\}_{i \in \mathcal{M}_{t}}$ is the set of measurements of the virtual landmarks observed at time $t$. The function $\Lambda(\cdot)$ is the information matrix updates of the estimated state distribution of the EIF-SLAM algorithm. These are done by first performing the time update, using (2.22a) for the point-approximation approach or (3.18) for the upper and lower bounds in the information-bound approach. The expected information gained from observing the virtual landmarks is then computed, using (3.10) for the point-approximation approach or (3.16) for the information-bound approach. Optionally, the detection probabilities, introduced in Chapter 4, could be included in the information-bound approach using (4.17). Lastly, the measurement update is performed by using (3.13) for the point-approximation approach or (3.19) for the information-bound approach. Note that, in general, the lower bound of the information matrix in the information-bound approach is inserted into $(3.20 \mathrm{~b})$ to obtain an upper approximation of the uncertainty of the platform position and orientation state estimates. This approximation is used to compute the performance measure $J(\cdot)$ in $(5.1)$.

By using a discretized state space, the problem in (5.1) can then be solved using an $\mathrm{A}^{*}$ graph-search algorithm, described in Section 2.2.2, adapted to beliefspace planning. An illustration of the proposed approach can be seen in Figure 5.1.

The complete modified $A^{*}$ algorithm can be found in Algorithm 5.1. The main steps involved can be summarized as:

1. Initialization: Initialize the priority queue $\mathcal{V}_{\text {open }}$ by adding the initial node to it (line 4 in Algorithm 5.1). 


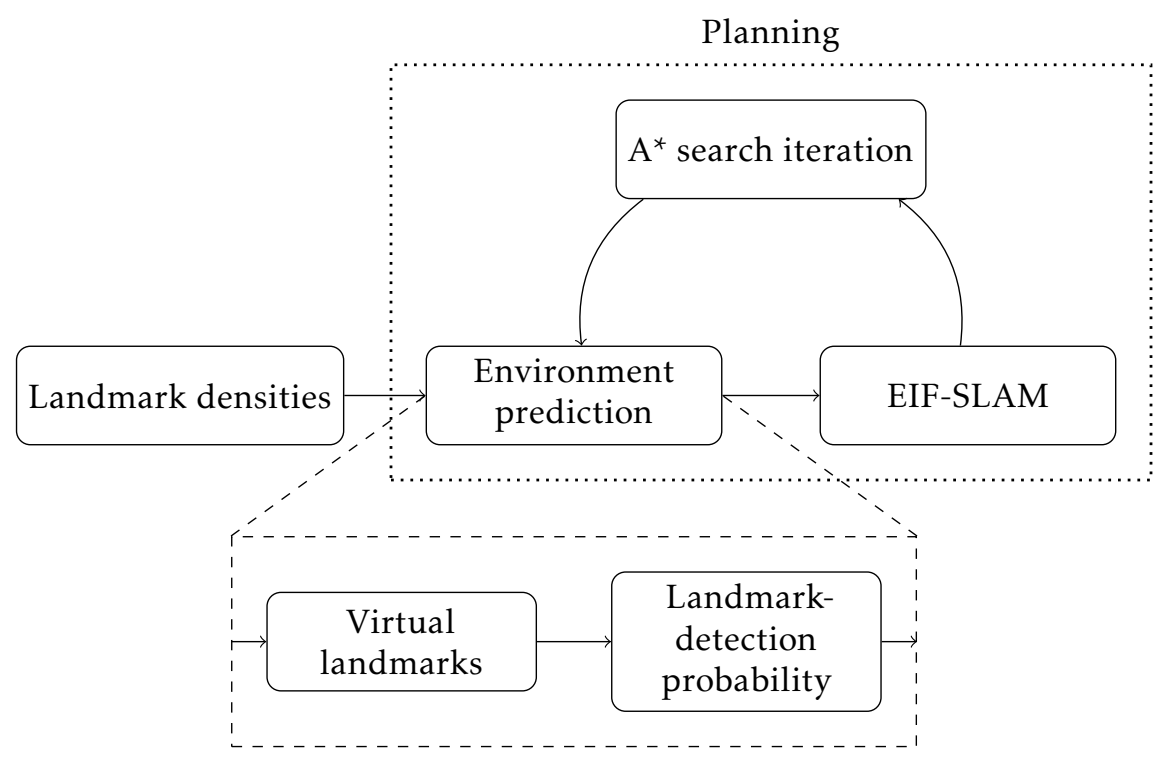

Figure 5.1: Illustration of the proposed planning problem for minimizing the uncertainty. The belief-space planning problem can be solved by first estimating the information gained from the environment using the virtual landmarks, then updating the state using the EIF-SLAM algorithm and lastly perform an $A^{*}$ search iteration.

2. Expansion: Pick a node from the queue and add it to the closed set $\mathcal{V}_{\text {closed }}$ (lines 6-7 in Algorithm 5.1).

3. EIF-SLAM update: For each possible action that leads to a successor, perform a time and measurement update to determine $\mathcal{I}_{t \mid t}$ for the successor nodes, using the virtual landmarks as described above, and compute the successor nodes priorities (lines 11-21 in Algorithm 5.1).

4. Insertion: Add the successor nodes to the priority queue $\mathcal{V}_{\text {open }}$, if the state is already in the priority queue with a higher priority, replace it with the successor node (lines 26-33 in Algorithm 5.1).

5. Check termination criterion: End the search if the goal set is reached (line 8 in Algorithm 5.1), otherwise go to step 2.

The path generated from the $\mathrm{A}^{*}$ algorithm is then obtained by backtracking through all parent nodes when the goal set is reached. 


\section{Algorithm 5.1: The $A^{*}$ algorithm adapted for virtual landmarks}

1: Input: An initial node $n_{\text {init }}$ containing the state $n_{\text {init }} \cdot \hat{x}=x_{\text {init }}$ and a goal set $\mathcal{X}_{\text {goal }}$

2: $\mathcal{V}_{\text {closed }}=\emptyset$

3: $\mathcal{V}_{\text {open }}=\emptyset$

4: $\mathcal{V}_{\text {open }} \cdot \operatorname{push}\left(n_{\text {init }}\right)$

5: while $\mathcal{V}_{\text {open }} \neq \emptyset$ do

6: $\quad n_{\text {current }} \leftarrow \mathcal{V}_{\text {open }} \cdot \operatorname{pop}()$

7: $\quad \mathcal{V}_{\text {closed }} \cdot \operatorname{push}\left(n_{\text {current }}\right)$

8: $\quad$ if $n_{\text {current }} . \hat{x} \in \mathcal{X}_{\text {goal }}$ then

9: $\quad$ return back_track $\left(n_{\text {current }}\right)$

10: end if

11: $\quad$ for $u_{t} \in \mathcal{U}\left(n_{\text {current }} . \hat{x}\right)$ do

12: $\quad \hat{x}_{t+1 \mid t+1} \leftarrow f\left(n_{\text {current }} . \hat{x}, u_{t}, 0\right)$

13: $\quad$ if $\hat{x}_{t+1 \mid t+1} \in \mathcal{V}_{\text {closed }}$ then

14: $\quad$ continue

15: $\quad$ end if

16: $\quad \mathcal{I}_{t+1 \mid t+1} \leftarrow \Lambda\left(n_{\text {current }} . \mathcal{I}, \hat{y}_{t}, n_{\text {current }} . \hat{x}, u_{t}\right)$

17: $\quad \hat{x}_{1: t+1} \leftarrow\left[n_{\text {current }} \cdot \hat{x}_{1: t} \hat{x}_{t+1 \mid t+1}\right]$

18: $\quad \mathcal{I}_{1: t+1} \leftarrow\left[n_{\text {current }} \cdot \mathcal{I}_{1: t} \mathcal{I}_{t+1 \mid t+1}\right]$

19: $\quad g_{t+1} \leftarrow J\left(\mathcal{I}_{1: t+1}, \hat{x}_{1: t+1}\right)$

20: $\quad n_{\text {child }} \cdot g \leftarrow g_{t+1}$

21: $\quad n_{\text {child }} \cdot$ priority $\leftarrow g_{t+1}+c\left(\hat{x}_{t+1 \mid t+1}, \mathcal{I}_{t+1 \mid t+1}\right)$

22: $\quad n_{\text {child }} \cdot \hat{x} \leftarrow \hat{x}_{t \mid t}$

23: $\quad n_{\text {child }} \cdot \hat{x}_{1: t} \leftarrow \hat{x}_{1: t+1}$

24: $\quad n_{\text {child }} \cdot \mathcal{I} \leftarrow \mathcal{I}_{t+1 \mid t+1}$

25: $\quad n_{\text {child }} \cdot \mathcal{I}_{1: t} \leftarrow \mathcal{I}_{1: t+1}$

26: $\quad$ if $\hat{x}_{t+1 \mid t+1} \notin \mathcal{V}_{\text {open }}$ then

27: $\quad \mathcal{V}_{\text {open }} \cdot \operatorname{push}\left(n_{\text {child }}\right)$

28: else

29: $\quad n^{\prime} \leftarrow \mathcal{V}_{\text {open }} \cdot \operatorname{get}\left(\hat{x}_{t+1 \mid t+1}\right)$

30: $\quad$ if $n_{\text {child }}$. priority $<n^{\prime}$. priority then

31: $\quad V_{\text {open }}$.replace $\left(n^{\prime}, n_{\text {child }}\right)$

32: $\quad$ end if

33: end if

34: end for

35: end while 


\subsection{Numerical evaluation}

In this section, the entire process shown in Figure 5.1 is evaluated in a 2D-setting. This is done in two steps. First, an evaluation comparing the path generation using the point-approximation approach and the information-bound approach is presented for two scenarios in an artificial environment. Then an evaluation of the information-bound approach, including the generated landmark densities and the landmark-detection probabilities, is presented for two forest scenarios. The generated paths are evaluated using Monte Carlo simulations.

\subsubsection{Implementation aspects}

During planning, the state space is discretized in the $x y$-plane, allowing only a subset $\mathcal{X}$ of the otherwise continuous set of positions for the platform. The discretized subspace, used during planning, is a uniform grid where the distance between each grid point is $20 \mathrm{~m}$ along the $x$ - and $y$-axis. The motion and measurement models described in Section 2.3.1 and Section 2.3.2 are used during all evaluations.

Let $\pi_{t}=\left[\pi_{t}^{x}, \pi_{t}^{y}\right]^{\top}$, where $\pi_{t}^{x}$ and $\pi_{t}^{y}$ denote the $x$ - and $y$-component, respectively, of $\pi_{t}$ in the parameter sequence $\left\{\pi_{t}\right\}_{t=1}^{T}$. These can be interpreted as the reference positions for the platform and are determined by the $\mathrm{A}^{*}$ algorithm during planing. During both the planning phase and motion execution, the control inputs to the platform are then determined by the simple policy $\pi(\cdot)$, defined as

$$
\pi\left(\hat{x}_{t \mid t}, \mathcal{I}_{t \mid t}, \pi_{t+1}\right)=\left[\begin{array}{c}
\operatorname{atan} 2\left(\pi_{t+1}^{y}-\hat{p}_{t \mid t}^{y}, \pi_{t+1}^{x}-\hat{p}_{t \mid t}^{x}\right)-\hat{p}_{t \mid t}^{\psi} \\
\left\|\left[\pi_{t+1}^{x}-\hat{p}_{t \mid t}^{x}, \pi_{t+1}^{y}-\hat{p}_{t \mid t}^{y}\right]^{\top}\right\|
\end{array}\right],
$$

where $\hat{p}_{t \mid t}^{x}$ and $\hat{p}_{t \mid t}^{y}$ are the estimated $x$ - and $y$-positions in $\hat{x}_{t \mid t}$ and $\hat{p}_{t \mid t}^{\psi}$ is the estimated orientation.

The chosen performance measure used during planning in all scenarios is the maximum trace of the covariance of the platform position and orientation estimates, described in Section 2.2.4, i.e.,

$$
J\left(\hat{x}_{0: T}, \mathcal{I}_{0: T}\right)=\max _{\tau \in[0: T]} \operatorname{tr}\left(P_{\tau \mid \tau}^{p}\right),
$$

where $P_{t \mid t}^{p}$ is the submatrix in $P_{t \mid t}$ related to the position and orientation estimates and $P_{t \mid t}=\mathcal{I}_{t \mid t}^{-1}$. Since no heuristic of this choice of performance measure is known, it is chosen as

$$
c\left(\hat{x}_{t \mid t}, \mathcal{I}_{t \mid t}\right)=0 \text {. }
$$

Note that, with this heuristic, the $\mathrm{A}^{*}$ algorithm is equivalent to Djikstra's algorithm. However, the $A^{*}$ algorithm allows for efficient planning with other performance measures, for which it is possible to find better heuristics, in the future. Furthermore, when using (5.3), there is a possibility that loops with a limited increase in cost-to-come exists. To avoid a situation where the search algorithm 
revisit the same points multiple times, the solution is restricted such that each point can only be visited once in the generated path.

For the information-bound approach, the upper and lower bounds $\overline{\mathcal{I}}_{t \mid t}$ and $\underline{I}_{t \mid t}$ on $\mathcal{I}_{t \mid t}$ are computed for each time step in the planning phase using (3.18) and (3.19). Note that, when the matrices $\overline{\mathcal{I}}_{t \mid t}$ and $\underline{I}_{t \mid t}$ are inverted to compute bounds on the covariance matrix $P_{t \mid t}$ using (3.20), the bounds on the true covariance will be underestimated due to the integral approximation introduced in (3.9), as shown in (3.36). As a result, the upper bound will only be an approximation of the true upper bound of the covariance matrix. These bounds are then used to calculate an approximate lower and upper bound of $J(\cdot)$. Furthermore, for the information-bound approach in all scenarios, the upper bound of the covariance matrix $P_{t \mid t}$ is used to calculate the cost-to-come used in the $\mathrm{A}^{*}$ algorithm during planning.

During evaluation, the generated performance measure is compared to a Monte Carlo estimate, computed from 100 Monte Carlo simulations for each scenario. In these Monte Carlo simulations, an autonomous platform is executing the planned path while performing SLAM with real landmarks using Algorithm 2.2, with different noise realizations for each simulation. The information matrices $\mathcal{I}_{t \mid t}$ from these simulations are then used to compute $\operatorname{tr}\left(P_{t \mid t}^{p}\right)$ for all $t \in\{1,2, \ldots, T\}$. The Monte Carlo estimate is then determined by averaging over all 100 values for each time step.

\subsubsection{Artificial environment}

The first scenario in the artificial environment highlights the principal path characteristics while the second shows the performance of the information-bound approach for a larger scenario.

It is assumed that the platform carries a range-bearing sensor, e.g., a LiDAR, with a maximum sensor range of $100 \mathrm{~m}$ and a $360^{\circ}$ field of view in both scenarios. In order to reduce the risk of the linearizations introduced in EIF-SLAM algorithm being inaccurate (Sim, 2005), the minimum sensor range $r_{\min }$ is set to

$$
r_{\min }=\max \left(10,1.96 \times \lambda_{\max }^{p}\right),
$$

where $\lambda_{\max }^{p}$ is the largest eigenvalue of the position covariance matrix. During motion execution, all landmarks within the maximum and minimum range are detected, occlusion is not considered. The process and measurement noise covariance matrices used in both scenarios are respectively

$$
Q=\left[\begin{array}{cc}
(4 \pi / 180)^{2} & 0 \\
0 & 5^{2}
\end{array}\right]
$$

and

$$
R=\left[\begin{array}{cc}
0.2^{2} & 0 \\
0 & (\pi / 180)^{2}
\end{array}\right]
$$

For each Monte Carlo simulation, a unique set of landmarks is sampled from the underlying densities. The dark and light green areas, as seen in Figure 5.2 
and Figure 5.4, contain $2 \cdot 10^{-5}$ landmarks $/ \mathrm{m}^{2}$ and $24 \cdot 10^{-5}$ landmarks $/ \mathrm{m}^{2}$, respectively.

\section{Scenario 1 - Path characteristics}

In the first scenario, the area is divided into subregions of sizes $10 \times 10 \mathrm{~m}^{2}$, where each subregion is approximated with $K=10$ sectors for the information-bound approach. The path and the first ten Monte Carlo simulations can be seen in Figure 5.2. The path generated with the information-bound approach has both more and larger curves than the path created with the point-approximation approach. Two advantages of these larger curves are identified:

- they facilitate local loop-closures as previously covered areas are regularly revisited, and

- subregions (and any possible landmarks) are viewed from more than one direction, thereby increasing their associated information.

The performance bounds of $J(\cdot)$ for the information-bound approach can be seen in Figure 5.3 as well as the Monte Carlo statistics. The figure also includes box plots illustrating the distribution of the performance measure for the simulations at different steps of the path. The mean of the performance measure for the different Monte Carlo simulations are within the calculated bounds, as well as the majority of the realizations. The outliers are a result of the process noise realizations moving the vehicle into the dark green areas where few landmarks exist. This thereby reduces the information gained compared to the prediction in the planning. This effect appears since the approach does not take realizations of the motion uncertainty into consideration. Addressing this issue is considered important in future developments.

The figure also shows the cost-to-come $g_{t}=J\left(\hat{x}_{0: t}, \mathcal{I}_{0: t}\right)$ for the point-approximation approach computed for each step along the path. This performance measure is worse than the performance bounds computed for the information-bound approach. As the point-approximation approach uses a point based estimate for the information matrix, i.e., the center point of each subregion, its accuracy is more sensitive to the size of the subregions. Although accurate for the first 20 steps, it seems to underestimate the information matrix in each step, as the estimated performance measure is higher than the actual uncertainty. This overestimated uncertainty makes newly observed areas less informative. As the minimum sensor range also increases with a higher position uncertainty, according to (5.5), the estimate quickly becomes inaccurate.

The information-bound approach also leads to longer paths than the pointapproximation approach. This is due to the chosen performance measure, where shorter paths are not favored if the covariance related to the platform position and orientation estimates is not reduced. Since, the point-approximation approach seems to underestimate the information gained from observations, it is less favorable to travel longer paths as the information from measurements cannot offset the added motion uncertainty, which leads to a higher position covariance. 


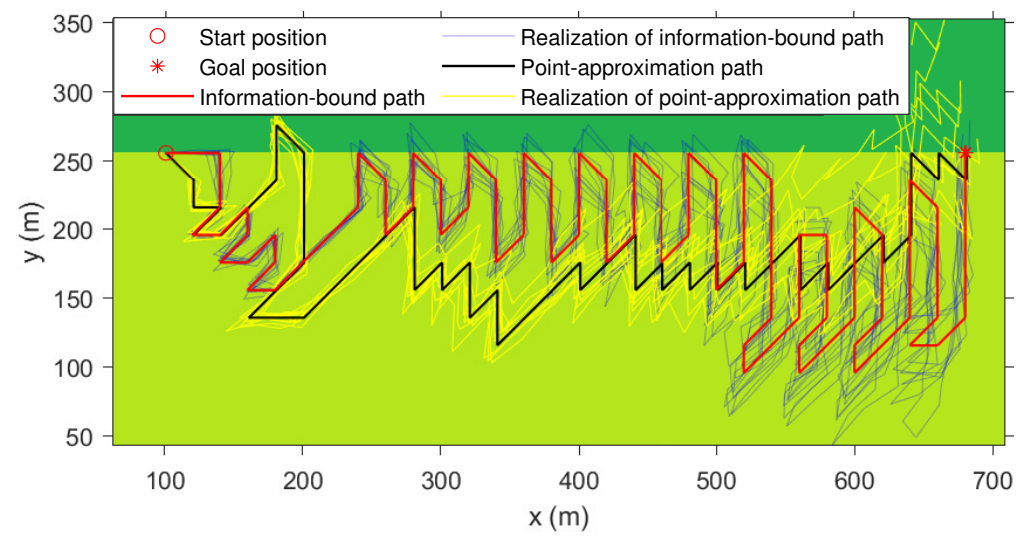

Figure 5.2: Illustration of the path of the information-bound approach (red) used in the Scenario 1, in Section 5.2.2, together with the path created with the point-approximation approach (black). The first ten Monte Carlo simulations for the information-bound approach (blue) and point-approximation approach (yellow) can also be seen.

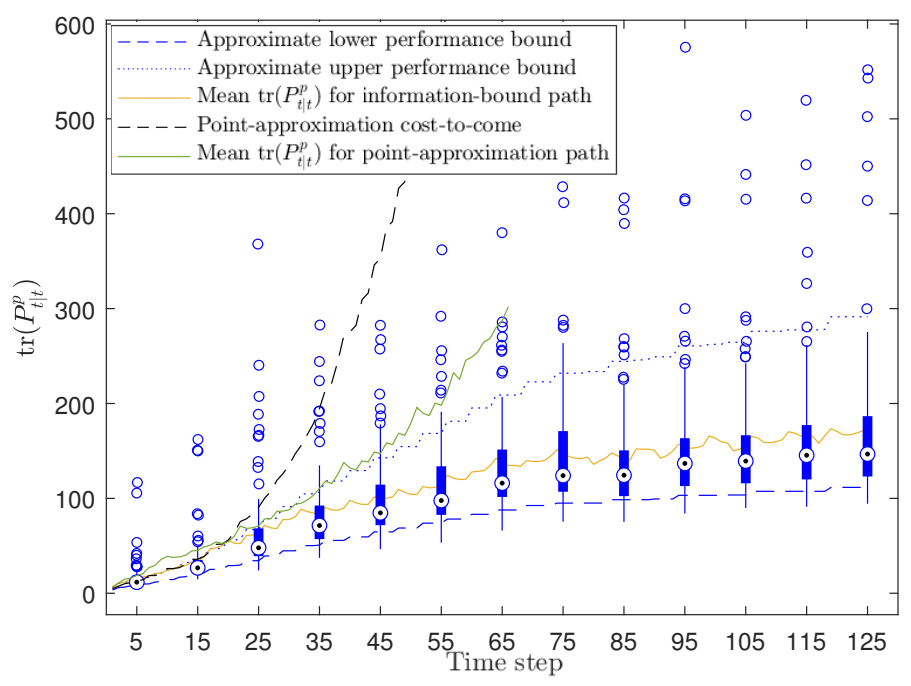

Figure 5.3: Approximate performance bounds of the information-bound approach (blue) and the cost-to-come of the point-approximation approach (black) for the scenario in Figure 5.2. The figure also shows the mean of 100 Monte Carlo estimates (orange and green). The blue boxplots show the distribution of the simulations for the information-bound approach, notice that the mean value is within the calculated bounds. 


\section{Scenario 2 - Large-scale test}

In the second scenario, the point-approximation approach and the informationbound approach are compared in a much larger region than in the first scenario. For computational efficiency, as the scenario is larger, the entire area is divided into subregions of size $25 \times 25 \mathrm{~m}^{2}$. Each subregion is approximated with $K=4$ sectors for the information-bound approach. The scenario includes a total of 2418 subregions.

The generated paths can be seen in Figure 5.4 and the performance bounds of the information-bound approach and the cost-to-come $g_{t}=J\left(\hat{x}_{0: t}, \mathcal{I}_{0: t}\right)$ of the point-approximation approach can be seen in Figure 5.5. The performance and characteristic of the paths are similar to those in the first scenario, where the performance of the point-approximation approach is still worse than the informationbound approach. The information-bound approach produces a sensible path where regions are revisited multiple times, which facilitates loop-closures, while avoiding the low density parts of the map. The mean of the performance measure of the information-bound approach is within the approximate bounds. However, it can be seen in the Figure 5.5 that a larger proportion of the Monte Carlo simulations is outside the bounds, compared to Figure 5.3. This is expected as the integral approximation used in the information-bound approach underestimates the true covariance matrix, as shown in (3.36). As more information is added with larger subregions $\Omega_{i}$, as discussed in Section 3.3.2, the upper performance bound becomes more inaccurate compared to the scenario in Figure 5.3 which has smaller subregions. Note that, the upper performance bound and the Monte Carlo estimate of the information-bound approach, in Figure 5.5, seem to grow in unison, and the bounds seem reasonable for all time steps along the path.

Implemented in Matlab and running on a laptop with a $2.9 \mathrm{GHz}$ Intel i7 processor, the information-bound approach ran at an average speed of $0.036 \mathrm{~s} /$ node expansions during planning in the second scenario, while the point-approximation ran at $0.034 \mathrm{~s} /$ node expansions under identical conditions. Note that, since the point-approximation approach produces a much shorter path, the total planning time was shorter for this approach.

\subsubsection{Planning in a forest environment}

In this part, paths generated using the information-bound approach are evaluated in two scenarios in a forest environment. This evaluation uses the landmarkdetection probabilities introduced in Chapter 4 and landmark densities generated as described in Section 3.1.1.

The platform's sensor is a LiDAR with an angular field of view of $\alpha_{F O V}=2 \pi$ and produces a point cloud with 16 rows and $N_{W}=2048$ columns. In both scenarios, approximate upper and lower bounds of the expected information gained from observing a subregion are computed using (4.17). The areas are divided into subregions of sizes $6.25 \times 6.25 \mathrm{~m}^{2}$, where each subregion is approximated with $K=5$ sectors. The process and measurement noise covariance matrices 


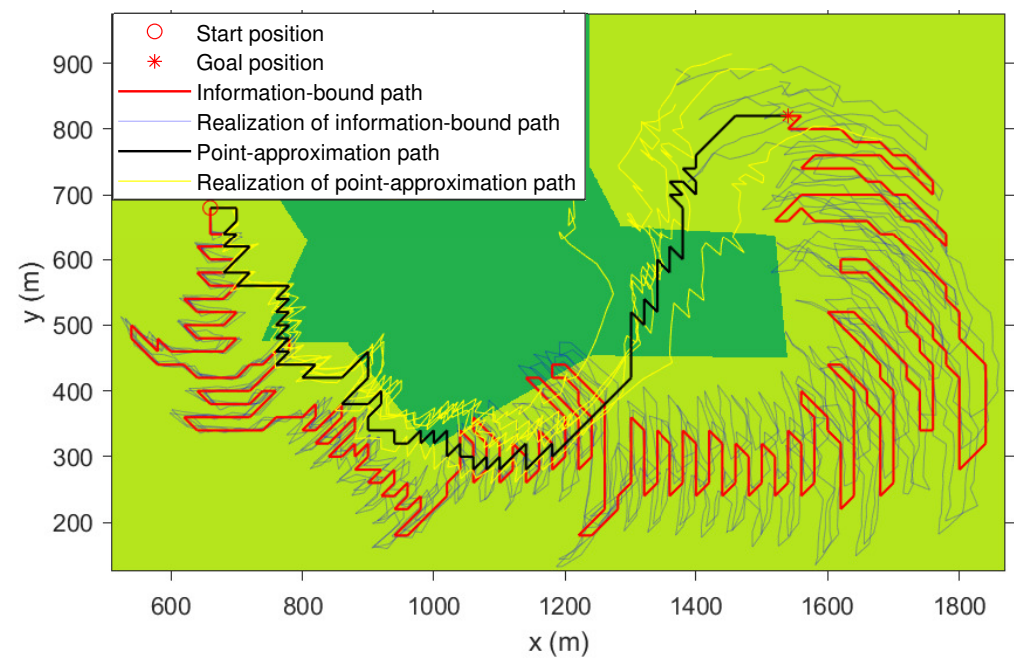

Figure 5.4: Illustration of the path of the information-bound approach (red) used in the Scenario 2, in Section 5.2.2, together with the path created with the point-approximation approach (black). The first ten Monte Carlo simulations for the information-bound approach (blue) and point-approximation approach (yellow) can also be seen.

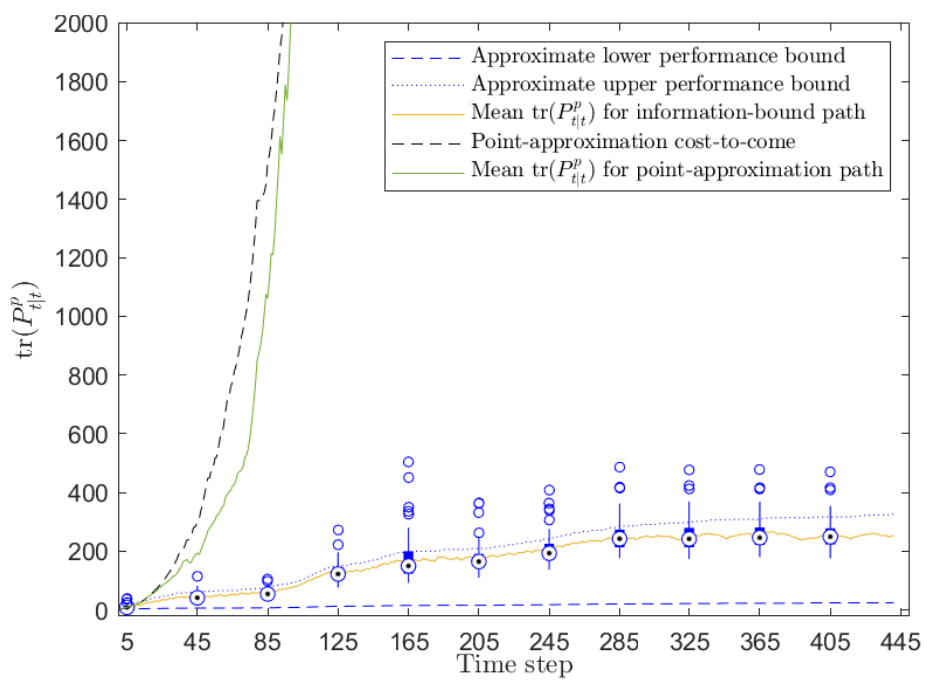

Figure 5.5: Approximate performance bounds of the information-bound approach (blue) and the cost-to-come of the point-approximation approach (black) for the scenario in Figure 5.4. The figure also shows the mean of 100 Monte Carlo estimates (orange and green). The blue boxplots show the distribution of the simulations for the information-bound approach, notice that the mean value is within the calculated bounds. 
used in both scenarios are respectively

$$
Q=\left[\begin{array}{cc}
(0.1 \pi / 180)^{2} & 0 \\
0 & 5^{2}
\end{array}\right]
$$

and

$$
R=\left[\begin{array}{cc}
2^{2} & 0 \\
0 & (4 \pi / 180)^{2}
\end{array}\right]
$$

The maximum range of the sensor is $75 \mathrm{~m}$ and the minimum range is

$$
r_{\min }=\max \left(1,1.96 \times \lambda_{\max }^{p}\right) .
$$

Note that, the maximum range is chosen as this is the practical limit of detecting trees using the modeled sensor and the landmark-detector algorithm.

In order to get landmark detections similar to a real environment in the Monte Carlo simulations used for evaluation, a point cloud is first created for each unique sensor position, as described in Section 4.6.1. The pole-landmark detector in Section 2.4 is then applied to the point cloud to generate the detections.

\section{Scenario 3}

In this scenario, an autonomous platform needs to reach the goal position by either traveling through a forested region or through an open area. The forested region contains a large number of landmarks but has a shorter view range, due occlusion. The open area has fewer landmarks but a longer view range. The generated path can be seen in Figure 5.6 while the performance bounds, computed with the information-bound approach, can be seen in Figure 5.7. The figures also show the shortest path between the start and goal position as reference.

The planned path moves the platform through the forested area with a shorter view range. Given the discussion in Section 4.6.3, this behavior is reasonable since the benefit of being able to see landmarks at longer distances is heavily outweighed by the lower probability that a landmark is large enough to be spotted at this distance. Instead, it is more favorable to travel in an area with large amounts of landmarks close to the sensor. The path has somewhat fewer curves when compared to the paths generated in Section 5.2. This is believed to be a consequence of the point cloud becoming less dense at longer ranges, which yields shorter detection ranges. Thus, large movements become more expensive, in terms of an increased position covariance, since these cannot be compensated for by observing more landmarks. This makes more direct paths more favorable.

\section{Scenario 4}

In this last scenario, the platform needs to travel across an open field in order to reach the goal state. The generated path can be seen in Figure 5.8, with the shortest path as reference. The computed performance bounds can be seen in Figure 5.9. 


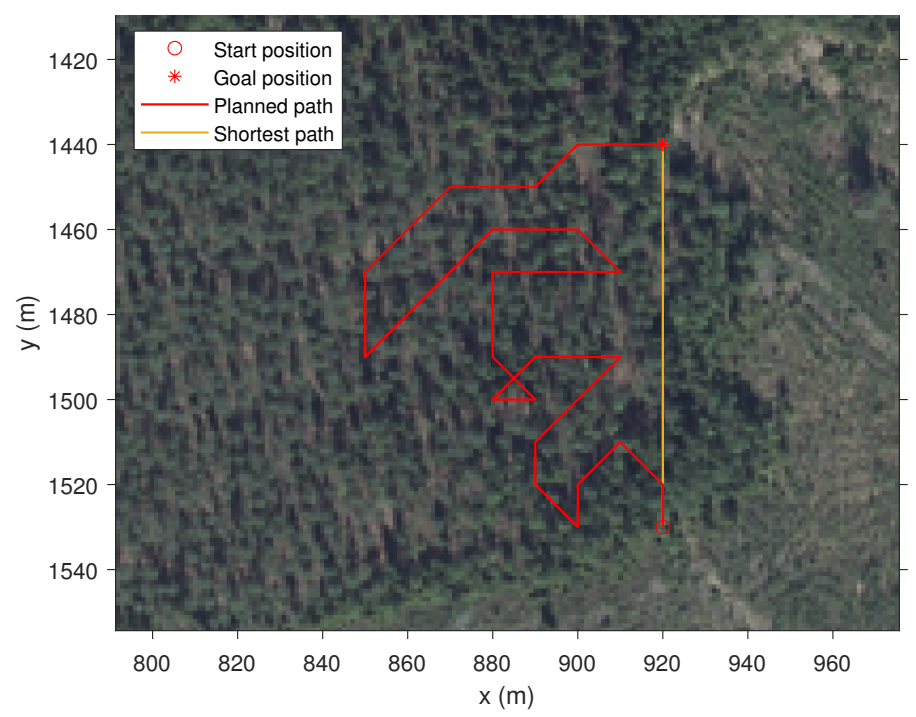

Figure 5.6: Illustration of the planned path (red) in Scenario 3, in Section 5.2.3, as well as the shortest path (orange) between the start and goal position.

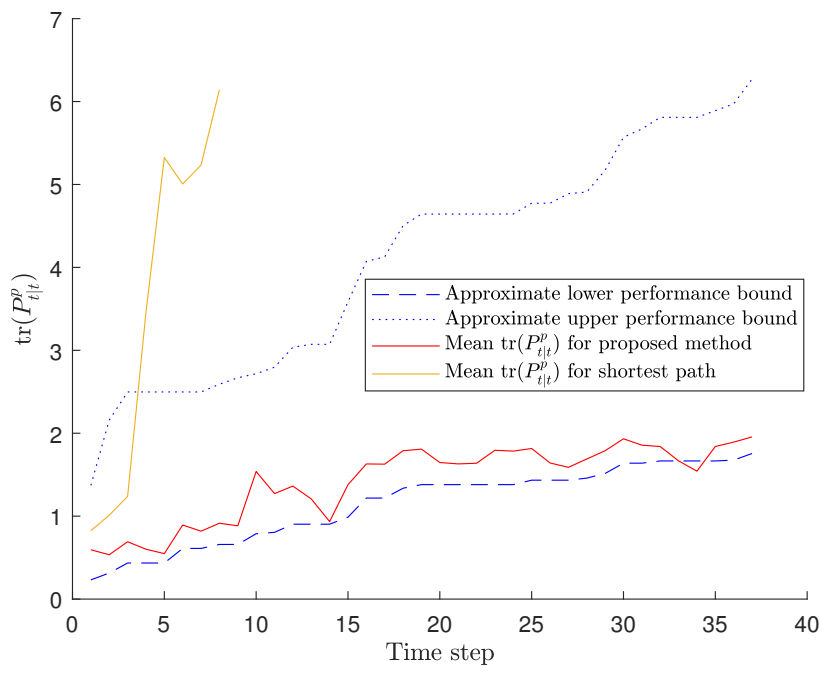

Figure 5.7: Approximate upper and lower performance bounds (dotted and dashed blue lines) together with the mean of the performance measure of 100 Monte Carlo realizations (red) for the scenarios in Figure 5.6. The figure also shows the mean of the performance measure of 100 Monte Carlo realizations for the shortest path between the start and goal position (orange). 


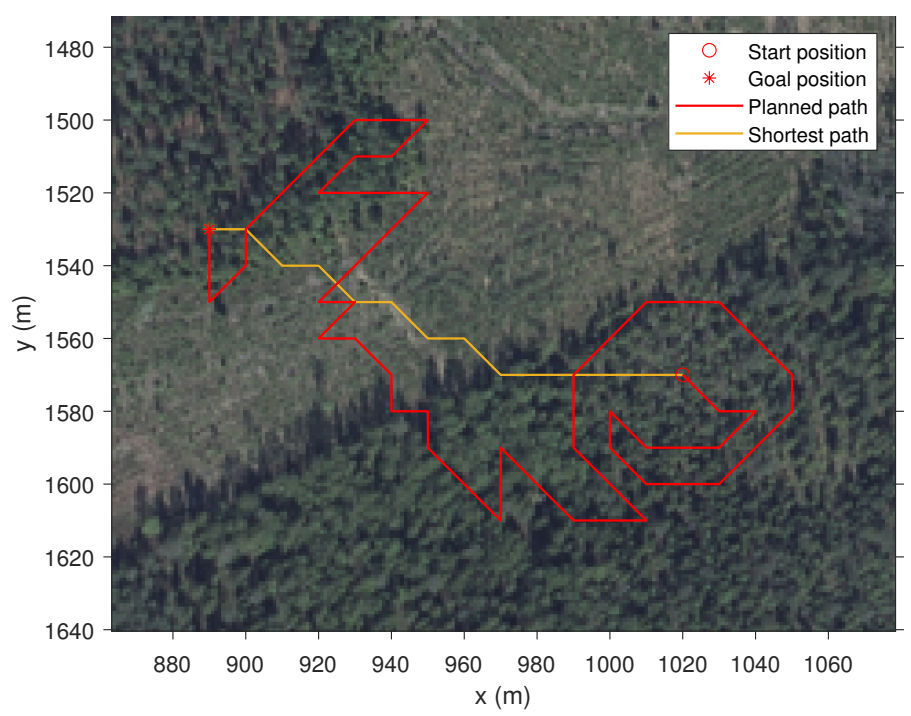

Figure 5.8: Illustration of the planned path (red) in Scenario 4, in Section 5.2.3, as well as the shortest path between the start and goal position (orange). In the scenario, an autonomous platform needs to reach the goal position by crossing an open area.

The trajectory begins with exploring the area towards the goal position but then returns and circles around the starting area before crossing the open area. By circling back to the starting area, after an initial exploration, a loop-closure is facilitated, similar to the paths created in Section 5.2. When previously observed areas are revisited, the correlation between newly observed subregions and the starting area increases. Since the area viewed before the first time update will be more informative than any later observed regions, increasing this correlation will also increase the position accuracy when any of these are viewed at a later state. While crossing the open area, the path becomes straighter yielding fewer measurement updates with little information gained. When the tree line on the other side of the open field comes into the field of view, the platform reverts to the previous strategy of making large curves as these are more favorable again.

The Monte Carlo estimate is within the bound except for the first part of the path, which is a result of a lower number of landmarks in the sensor field of view compared to the underlying density. Furthermore, the Monte Carlo estimate of the proposed approach is much smaller than the Monte Carlo estimate for the shortest path used as reference. Hence, a reduction in the position uncertainty has been attained. 


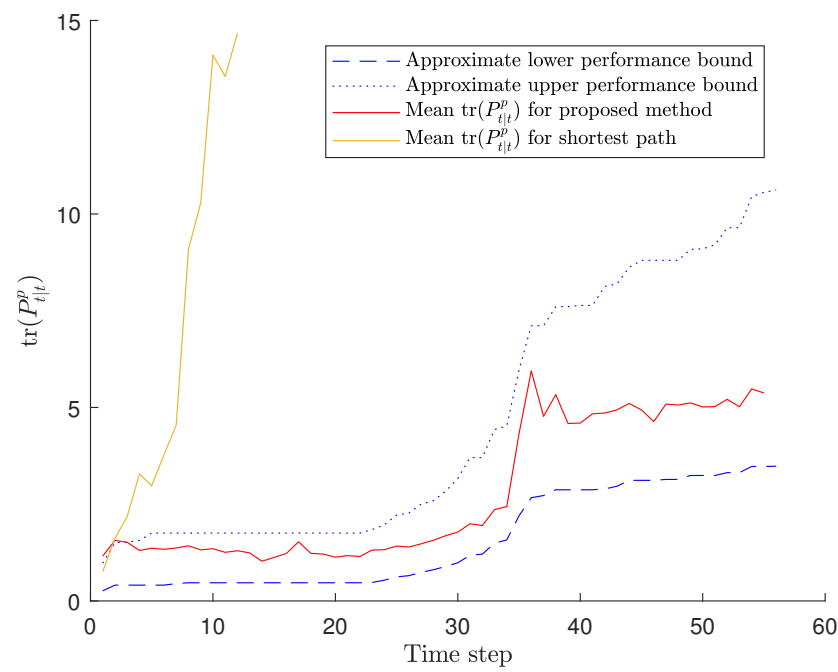

Figure 5.9: Approximate upper and lower performance bounds (dotted and dashed blue lines) together with the mean of the performance measure of 100 Monte Carlo realizations (red) for the scenarios in Figure 5.8. The figure also shows the mean of the performance measure of 100 Monte Carlo realizations for the shortest path between the start and goal position (orange). 


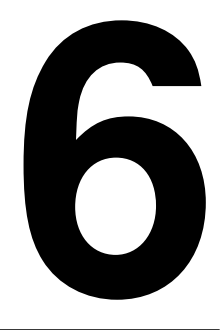

\section{Concluding remarks}

This chapter concludes the thesis with a summary of the results and discussion of some possible future research topics.

\subsection{Conclusion}

The aim of this work is to develop a model for determining the expected information gained from observing a previously unexplored area without explicit knowledge of landmark positions.

The concept of virtual landmarks was introduced in order to represent the information that can be expected in unexplored regions during planning. This is an improvement compared to common practice where this information is often ignored. The information gained from observing these virtual landmarks was then determined based upon two factors. The factors relate to the existence of real landmarks in the virtual landmarks' immediate surrounding and the probability of detecting real landmarks close to the virtual landmark. Two approaches for computing this gained information were introduced. The first approach is a general approach, applicable to most measurement models, while the second approach introduced a refinement for range-bearing measurement models. Using these, it was shown how an approximation of the state covariance can be computed through landmark-based SLAM algorithms, without explicit knowledge of the landmarks' positions. This state covariance is used to describe the quality of the state estimate, where a larger covariance corresponds to a more uncertain state estimate.

Furthermore, it has been shown how the detection probability of landmarks can be computed for range-bearing LiDAR measurements and included in the planning problem. The detection probability was modeled from a real landmark detector used in SLAM and was computed by taking the probability of occlusion, 
the sensor properties, and properties of the landmark detector into account. This is considered to be an important contribution in order to be able to use the developed approach in a real world scenario as it is otherwise often overlooked in simulation studies.

Moreover, it was shown how landmark densities can be computed from prior data from airborne LiDAR measurements for a forest scenario. These densities were then used to compute the factor related to the existence of real landmarks in the virtual landmarks' immediate surrounding.

Lastly, all of these contributions were combined into a path-planning approach based upon a modified $A^{*}$ graph-search algorithm. With this algorithm, a solution to the motion-planning problem was computed without explicit knowledge of landmark positions. The state estimate was simulated during planning in order to predict the actual state estimate computed with SLAM during motion execution.

The developed approach is by no means a general solution for minimumuncertainty motion-planning with unknown landmarks. Several approximations are made which potentially contribute to some error or deviation from optimality. However, the contributions presented in this thesis give some insight on how to solve this problem for specific situations. The proposed approach managed to generate paths which reduced the chosen measure of uncertainty compared to multiple references during execution, for a range-bearing sensor in a forest scenario without prior knowledge of landmarks. How this approach performs in an evaluation in the real world remains to be tested but simulations show promising results.

\subsection{Future work}

Three main areas of improvement have been identified, these are state estimation, integration into belief-space planning algorithms, and inclusion of external effects.

The aim of this work has been to introduce a way of representing the information in unexplored regions of the environment. As such, the only considered procedure for state estimation is the EIF-SLAM algorithm. This approach does not take negative information, i.e., that absence of landmarks in an area, into account. For instance, while traveling in a dense forest, reaching an open field without any landmarks can sometimes be more informative than the landmarks themselves. Such information can be utilized by using a particle filter (Gustafsson, 2010a) for state estimation. Furthermore, the multi-modal property of the particle filter could also be useful in order to limit the effect of highly nonlinear situations, where the linearization of the measurement and motion models in EIF-SLAM become inaccurate.

Due to the introduced integral approximation, only an approximate upper bound on the true position uncertainty is presented in the information-bound approach. However, it is considered important to further investigate how to compute a true upper bound in future work. 
The planning algorithms considered in this work are limited to simple graphsearch algorithms such as $\mathrm{A}^{*}$ and Dijkstra's algorithm. The assumption of unknown landmarks generally makes it difficult to use belief-space planning algorithms in a meaningful way, since only the time update can be performed and no corrections can be made using measurements. In the belief road map (Prentice and Roy, 2009), the state covariance matrix updates are divided into factors which are precomputed for all nodes in a graph. Under the assumption of completely unknown landmarks, these calculations cannot be performed. However, by using the introduced virtual landmarks, these computations should be possible, which would result in a highly efficient search algorithm. This should be an interesting future research topic. This procedure should also extend to other algorithms such as the rapidly exploring belief trees (Bry and Roy, 2011).

Lastly, another source of uncertainty that affects the motion in real applications is the terrain properties. Wet, loose or sloping terrain can make a ground moving platform slip, slide or get stuck. Currently all of these effects are assumed to be captured by the motion noise terms introduced in the EIF-SLAM algorithm. However, it should be possible to describe and estimate these motion uncertainties in more detail using prior data. This is believed to be an underdeveloped topic in belief-space planning and progress could possibly be made by combining approaches from the field of, e.g., terramechanics (Wong, 1989). 



\section{Bibliography}

R. Angevine, J. K. Warden, R. Keller, and C. Frye. Learning lessons from the Ukraine conflict. Technical Report NS D-10367, Institute for Defense Analyses, Alexandria, VA, USA, May 2019.

K. J. Åström. Introduction to stochastic control theory. Academic Press, New York, NY, USA, 1970.

T. Bailey and H. Durrant-Whyte. Simultaneous localization and mapping (SLAM): part II. IEEE Robotics and Automation Magazine, 13(3):108-117, Sept. 2006.

A. Ben-Israel. A Newton-Raphson method for the solution of systems of equations. Journal of Mathematical Analysis and Applications, 15(2):243-252, 1966.

G. E. Box. Science and statistics. Journal of the American Statistical Association, 71(356):791-799, 1976.

S. Boyd and L. Vandenberghe. Convex Optimization. Cambridge University Press, New York, NY, USA, 2004.

G. Bresson, Z. Alsayed, L. Yu, and S. Glaser. Simultaneous localization and mapping: A survey of current trends in autonomous driving. IEEE Transactions on Intelligent Vehicles, 2(3):194-220, Sept. 2017.

A. Bry and N. Roy. Rapidly-exploring random belief trees for motion planning under uncertainty. In Proceedings of the 2011 IEEE International Conference on Robotics and Automation (ICRA), pages 723-730, Shanghai, China, May 2011. IEEE.

S. M. Chaves, A. Kim, and R. M. Eustice. Opportunistic sampling-based planning for active visual SLAM. In Proceedings of the IEEE/RSJ International Conference on Intelligent Robots and Systems (IROS), pages 3073-3080, Chicago, IL, USA, 2014.

Y. Chen, S. Huang, and R. Fitch. Active SLAM for mobile robots with area coverage and obstacle avoidance. IEEE/ASME Transactions on Mechatronics, 25(3): 1182-1192, 2020. 
E. W. Dijkstra. A note on two problems in connexion with graphs. Numerische mathematik, 1(1):269-271, 1959.

H. Durrant-Whyte and T. Bailey. Simultaneous localization and mapping (SLAM): part I. IEEE Robotics and Automation Magazine, 13(2):99-110, June 2006.

C. Ericson. Real-time collision detection. CRC Press, 2004.

N. Evestedt, O. Ljungqvist, and D. Axehill. Motion planning for a reversing general 2-trailer configuration using closed-loop RRT. In Proceedings of the IEEE/RSJ International Conference on Intelligent Robots and Systems (IROS), pages 3690-3697, Daejeon, Korea, Oct. 2016.

K. M. Frey, T. J. Steiner, and J. P. How. Towards online observabilityaware trajectory optimization for landmark-based estimators. arXiv preprint arXiv:1908.03790, 2019.

F. Gustafsson. Particle filter theory and practice with positioning applications. IEEE Aerospace and Electronic Systems Magazine, 25(7):53-82, 2010a.

F. Gustafsson. Statistical sensor fusion. Studentlitteratur, $2010 \mathrm{~b}$.

P. E. Hart, N. J. Nilsson, and B. Raphael. A formal basis for the heuristic determination of minimum cost paths. IEEE Transactions on Systems Science and Cybernetics, 4(2):100-107, 1968.

V. Indelman, L. Carlone, and F. Dellaert. Planning in the continuous domain: A generalized belief space approach for autonomous navigation in unknown environments. International Journal of Robotics Research, 34(7):849-882, 2015.

L. P. Kaelbling, M. L. Littman, and A. R. Cassandra. Planning and acting in partially observable stochastic domains. Artificial intelligence, 101(1-2):99_ 134, 1998.

T. Kailath, A. H. Sayed, and B. Hassibi. Linear Estimation. Prentice Hall, New Jersey, 2000.

R. E. Kalman. A new approach to linear filtering and prediction problems. Transactions of the ASME, Journal of Basic Engineering, 82(1):35-45, Mar. 1960.

Y. Kuwata, J. Teo, G. Fiore, S. Karaman, E. Frazzoli, and J. P. How. Real-time motion planning with applications to autonomous urban driving. IEEE Transactions on Control Systems Technology, 17(5):1105-1118, 2009.

M. Labbé and F. Michaud. Long-term online multi-session graph-based SPLAM with memory management. Autonomous Robots, 42(6):1133-1150, 2018.

Lantmäteriet. Orphoto / Lantmäteriet, Jan. 2020. URL https: //www. lantmateriet.se/en/maps-and-geographic-information/ geodataprodukter/produktlista/orthophoto/. 
S. M. LaValle. Planning algorithms. Cambridge university press, New York, NY, USA, 2006.

C. Leung, S. Huang, and G. Dissanayake. Active SLAM using model predictive control and attractor based exploration. In Proceedings of the IEEE/RSJ International Conference on Intelligent Robots and Systems (IROS), pages 50265031, Beijing, China, Nov. 2006.

C. Leung, S. Huang, and G. Dissanayake. Active SLAM in structured environments. In Proceedings of the 2008 IEEE International Conference on Robotics and Automation (ICRA), pages 1898-1903, Pasadena, CA, USA, 2008.

H. W. Lilliefors. On the Kolmogorov-Smirnov test for normality with mean and variance unknown. Journal of the American Statistical Association, 62(318): 399-402, 1967.

T.-Y. Lin, P. Goyal, R. Girshick, K. He, and P. Dollár. Focal loss for dense object detection. In Proceedings of the IEEE International Conference on Computer Vision, pages 2980-2988, Venice, Italy, 2017.

P. C. Mahalanobis. On the generalized distance in statistics. In Proceedings of the National Institute of Science of India, volume 2, 1936.

S. Miller, Z. Harris, and E. Chong. Coordinated guidance of autonomous UAVs via nominal belief-state optimization. In Proceedings of the American Control Conference, pages 2811 - 2818, St. Louis, USA, July 2009.

D. C. Montgomery. Design and analysis of experiments. John Wiley \& Sons, Inc., 2009.

F. Näsström, F. Bissmarck, V. Deleskog, F. Hemström, M. Holmberg, J. Karlholm, J. Nordlöf, J. Nygårds, K.-G. Stenborg, and N. Wadströmer. Intelligent spaning 2016-2018: Slutrapport. Technical report, FOI, 2018.

K. Nielsen. Robust LIDAR-Based Localization in Underground Mines. Linköping University Electronic Press, 2021.

J. Nocedal and S. J. Wright. Numerical optimization. Springer, New York, NY, USA, 1999.

J. Nordlöf, G. Hendeby, and D. Axehill. Belief space planning using landmark density information. In IEEE Proceedings of the 23rd International Conference on Information Fusion (FUSION), pages 1-8, Rustenburg, South Africa, July 2020.

J. Nordlöf, G. Hendeby, and D. Axehill. Improved virtual landmark approximation of belief-space planning. In IEEE Proceedings of the 24th International Conference on Information Fusion (FUSION), pages 1-8, Rustenburg, South Africa, Nov. 2021. 
F. W. Olver, D. W. Lozier, R. F. Boisvert, and C. W. Clark. NIST Handbook of Mathematical Functions. Cambridge university press, New York, NY, USA, 2010.

Ouster, Inc. High-resolution OS1 lidar sensor: robotics, trucking, mapping | Ouster, 2021. URL https://ouster.com/products/scanning-lidar/ osi-sensor/.

C. H. Papadimitriou and J. N. Tsitsiklis. The complexity of Markov decision processes. Mathematics of operations research, 12(3):441-450, 1987.

R. Platt, R. Tedrake, L. Kaelbling, and T. Lozano-Perez. Belief space planning assuming maximum likelihood observations. In Robotics: Science and Systems VI, June 2010.

S. Prentice and N. Roy. The belief roadmap: Efficient planning in belief space by factoring the covariance. International Journal of Robotics Research, 28(11-12): 1448-1465, 2009.

F. Pukelsheim. Optimal design of experiments. SIAM, 2006.

M. L. Rodríguez-Arévalo, J. Neira, and J. A. Castellanos. On the importance of uncertainty representation in active SLAM. IEEE Transactions on Robotics, 34 (3):829-834, 2018.

S. Russell and P. Norvig. Artificial Intelligence: A Modern Approach, volume 82. Prentice Hall, 2003.

S. Särkkä. Bayesian filtering and smoothing. Cambridge University Press, 2013.

A. Schaefer, L. Luft, and W. Burgard. An analytical lidar sensor model based on ray path information. IEEE Transactions on Robotics and Automation, 2(3): 1405-1412, 2017.

A. Schaefer, D. Büscher, J. Vertens, L. Luft, and W. Burgard. Long-term urban vehicle localization using pole landmarks extracted from 3-d lidar scans. In Proceedings of the European Conference on Mobile Robots (ECMR), pages 1-7, Prague, Czech Republic, 2019. IEEE.

R. Schirmer, P. Biber, and C. Stachniss. Efficient path planning in belief space for safe navigation. In Proceedings of the IEEE/RSJ International Conference on Intelligent Robots and Systems (IROS), Vancouver, BC, Canada, Sept. 2017.

J. Siipilehto and L. Mehtätalo. Parameter recovery vs. parameter prediction for the Weibull distribution validated for Scots pine stands in Finland. Silva Fennica, 47(4), 2013.

R. Sim. Stabilizing information-driven exploration for bearings-only SLAM using range gating. In Proceedings of the IEEE/RSJ International Conference on Intelligent Robots and Systems (IROS), Edmonton, Canada, Aug. 2005. 
Skogsstyrelsen. Skogsstyrelsen - skoglig grunddata, 2021. URL https://www.skogsstyrelsen.se/sjalvservice/karttjanster/ skogliga-grunddata/.

E. D. Sontag. Mathematical control theory. Texts in Applied Mathematics. Springer, New York, NY, USA, 2 edition, 2013.

S. Thrun, Y. Liu, D. Koller, A. Y. Ng, Z. Ghahramani, and H. Durrant-Whyte. Simultaneous localization and mapping with sparse extended information filters. International Journal of Robotics Research, 23(7-8):693-716, Aug. 2004.

R. Valencia and J. Andrade-Cetto. Path planning in belief space with pose SLAM. In Mapping, Planning and Exploration with Pose SLAM, pages 53-87. Springer, 2018.

J. van den Berg, S. Patil, and R. Alterovitz. Motion planning under uncertainty using iterative local optimization in belief space. International Journal of Robotics Research, 31(11):1263-1278, 2012.

C. F. Van Loan and G. H. Golub. Matrix Computations (3rd Ed.). The Johns Hopkins University Press, Baltimore, MD, USA, 1996.

W. Weibull. A statistical distribution function of wide applicability. Journal of Applied Mechanics, 18(3):293-297, 1951.

B. G. Weinstein, S. Marconi, S. Bohlman, A. Zare, and E. White. Individual treecrown detection in RGB imagery using semi-supervised deep learning neural networks. Remote Sensing, 11(11):1309, 2019a.

B. G. Weinstein, S. Marconi, S. A. Bohlman, A. Zare, and E. P. White. Geographic generalization in airborne RGB deep learning tree detection. bioRxiv, 2019b.

J. Wojtanowski, M. Zygmunt, M. Kaszczuk, Z. Mierczyk, and M. Muzal. Comparison of $905 \mathrm{~nm}$ and $1550 \mathrm{~nm}$ semiconductor laser rangefinders' performance deterioration due to adverse environmental conditions. Opto-Electronics Review, 22(3):183-190, 2014.

Wolfram Research, Inc. Mathematica, Version 12.0, 2019.

J. Y. Wong. Terramechanics and off-road vehicles. Elsevier, 1989.

K. M. Wurm, C. Stachniss, and G. Grisetti. Bridging the gap between feature-and grid-based SLAM. Robotics and Autonomous Systems, 58(2):140-148, 2010.

C. Yang, L. Kaplan, and E. Blasch. Performance measures of covariance and information matrices in resource management for target state estimation. IEEE Transactions on Aerospace and Electronic Systems, 48(3):2594-2613, 2012.

H. Yang and S. Y. J. Lim. Anytime RRBT for handling uncertainty and dynamic objects. In Proceedings of the IEEE/RSJ International Conference on Intelligent Robots and Systems (IROS), Daejeon, Korea, Oct. 2016. 


\section{Licentiate Theses \\ Division of Automatic Control \\ Linköping University}

P. Andersson: Adaptive Forgetting through Multiple Models and Adaptive Control of Car Dynamics. Thesis No. 15, 1983.

B. Wahlberg: On Model Simplification in System Identification. Thesis No. 47, 1985.

A. Isaksson: Identification of Time Varying Systems and Applications of System Identification to Signal Processing. Thesis No. 75, 1986.

G. Malmberg: A Study of Adaptive Control Missiles. Thesis No. 76, 1986.

S. Gunnarsson: On the Mean Square Error of Transfer Function Estimates with Applications to Control. Thesis No. 90, 1986.

M. Viberg: On the Adaptive Array Problem. Thesis No. 117, 1987.

K. Ståhl: On the Frequency Domain Analysis of Nonlinear Systems. Thesis No. 137, 1988.

A. Skeppstedt: Construction of Composite Models from Large Data-Sets. Thesis No. 149, 1988.

P. A. J. Nagy: MaMiS: A Programming Environment for Numeric/Symbolic Data Processing. Thesis No. 153, 1988.

K. Forsman: Applications of Constructive Algebra to Control Problems. Thesis No. 231, 1990.

I. Klein: Planning for a Class of Sequential Control Problems. Thesis No. 234, 1990.

F. Gustafsson: Optimal Segmentation of Linear Regression Parameters. Thesis No. 246, 1990.

H. Hjalmarsson: On Estimation of Model Quality in System Identification. Thesis No. 251, 1990.

S. Andersson: Sensor Array Processing; Application to Mobile Communication Systems and Dimension Reduction. Thesis No. 255, 1990.

K. Wang Chen: Observability and Invertibility of Nonlinear Systems: A Differential Algebraic Approach. Thesis No. 282, 1991.

J. Sjöberg: Regularization Issues in Neural Network Models of Dynamical Systems. Thesis No. 366, 1993.

P. Pucar: Segmentation of Laser Range Radar Images Using Hidden Markov Field Models. Thesis No. 403, 1993.

H. Fortell: Volterra and Algebraic Approaches to the Zero Dynamics. Thesis No. 438, 1994.

T. McKelvey: On State-Space Models in System Identification. Thesis No. 447, 1994.

T. Andersson: Concepts and Algorithms for Non-Linear System Identifiability. Thesis No. 448, 1994.

P. Lindskog: Algorithms and Tools for System Identification Using Prior Knowledge. Thesis No. 456, 1994.

J. Plantin: Algebraic Methods for Verification and Control of Discrete Event Dynamic Systems. Thesis No. 501, 1995.

J. Gunnarsson: On Modeling of Discrete Event Dynamic Systems, Using Symbolic Algebraic Methods. Thesis No. 502, 1995.

A. Ericsson: Fast Power Control to Counteract Rayleigh Fading in Cellular Radio Systems. Thesis No. 527, 1995.

M. Jirstrand: Algebraic Methods for Modeling and Design in Control. Thesis No. 540, 1996.

K. Edström: Simulation of Mode Switching Systems Using Switched Bond Graphs. Thesis No. 586, 1996. 
J. Palmqvist: On Integrity Monitoring of Integrated Navigation Systems. Thesis No. 600, 1997.

A. Stenman: Just-in-Time Models with Applications to Dynamical Systems. Thesis No. 601, 1997.

M. Andersson: Experimental Design and Updating of Finite Element Models. Thesis No. 611, 1997.

U. Forssell: Properties and Usage of Closed-Loop Identification Methods. Thesis No. 641, 1997.

M. Larsson: On Modeling and Diagnosis of Discrete Event Dynamic systems. Thesis No. 648, 1997.

N. Bergman: Bayesian Inference in Terrain Navigation. Thesis No. 649, 1997.

V. Einarsson: On Verification of Switched Systems Using Abstractions. Thesis No. 705, 1998.

J. Blom, F. Gunnarsson: Power Control in Cellular Radio Systems. Thesis No. 706, 1998.

P. Spångéus: Hybrid Control using LP and LMI methods - Some Applications. Thesis No. 724, 1998.

M. Norrlöf: On Analysis and Implementation of Iterative Learning Control. Thesis No. 727, 1998.

A. Hagenblad: Aspects of the Identification of Wiener Models. Thesis No. 793, 1999.

F. Tjärnström: Quality Estimation of Approximate Models. Thesis No. 810, 2000.

C. Carlsson: Vehicle Size and Orientation Estimation Using Geometric Fitting. Thesis No. 840, 2000.

J. Löfberg: Linear Model Predictive Control: Stability and Robustness. Thesis No. 866, 2001.

O. Härkegård: Flight Control Design Using Backstepping. Thesis No. 875, 2001.

J. Elbornsson: Equalization of Distortion in A/D Converters. Thesis No. 883, 2001.

J. Roll: Robust Verification and Identification of Piecewise Affine Systems. Thesis No. 899, 2001.

I. Lind: Regressor Selection in System Identification using ANOVA. Thesis No. 921, 2001.

R. Karlsson: Simulation Based Methods for Target Tracking. Thesis No. 930, 2002.

P.-J. Nordlund: Sequential Monte Carlo Filters and Integrated Navigation. Thesis No. 945, 2002.

M. Östring: Identification, Diagnosis, and Control of a Flexible Robot Arm. Thesis No. 948, 2002.

C. Olsson: Active Engine Vibration Isolation using Feedback Control. Thesis No. 968, 2002.

J. Jansson: Tracking and Decision Making for Automotive Collision Avoidance. Thesis No. 965, 2002.

N. Persson: Event Based Sampling with Application to Spectral Estimation. Thesis No. 981, 2002.

D. Lindgren: Subspace Selection Techniques for Classification Problems. Thesis No. 995, 2002.

E. Geijer Lundin: Uplink Load in CDMA Cellular Systems. Thesis No. 1045, 2003.

M. Enqvist: Some Results on Linear Models of Nonlinear Systems. Thesis No. 1046, 2003.

T. Schön: On Computational Methods for Nonlinear Estimation. Thesis No. 1047, 2003.

F. Gunnarsson: On Modeling and Control of Network Queue Dynamics. Thesis No. 1048, 2003.

S. Björklund: A Survey and Comparison of Time-Delay Estimation Methods in Linear Systems. Thesis No. 1061, 2003. 
M. Gerdin: Parameter Estimation in Linear Descriptor Systems. Thesis No. 1085, 2004.

A. Eidehall: An Automotive Lane Guidance System. Thesis No. 1122, 2004.

E. Wernholt: On Multivariable and Nonlinear Identification of Industrial Robots. Thesis No. 1131, 2004.

J. Gillberg: Methods for Frequency Domain Estimation of Continuous-Time Models. Thesis No. 1133, 2004.

G. Hendeby: Fundamental Estimation and Detection Limits in Linear Non-Gaussian Systems. Thesis No. 1199, 2005.

D. Axehill: Applications of Integer Quadratic Programming in Control and Communication. Thesis No. 1218, 2005.

J. Sjöberg: Some Results On Optimal Control for Nonlinear Descriptor Systems. Thesis No. 1227, 2006.

D. Törnqvist: Statistical Fault Detection with Applications to IMU Disturbances. Thesis No. 1258, 2006.

H. Tidefelt: Structural algorithms and perturbations in differential-algebraic equations. Thesis No. 1318, 2007.

S. Moberg: On Modeling and Control of Flexible Manipulators. Thesis No. 1336, 2007.

J. Wallén: On Kinematic Modelling and Iterative Learning Control of Industrial Robots. Thesis No. 1343, 2008.

J. Harju Johansson: A Structure Utilizing Inexact Primal-Dual Interior-Point Method for Analysis of Linear Differential Inclusions. Thesis No. 1367, 2008.

J. D. Hol: Pose Estimation and Calibration Algorithms for Vision and Inertial Sensors. Thesis No. 1370, 2008.

H. Ohlsson: Regression on Manifolds with Implications for System Identification. Thesis No. 1382, 2008.

D. Ankelhed: On low order controller synthesis using rational constraints. Thesis No. 1398, 2009.

P. Skoglar: Planning Methods for Aerial Exploration and Ground Target Tracking. Thesis No. 1420, 2009.

C. Lundquist: Automotive Sensor Fusion for Situation Awareness. Thesis No. 1422, 2009.

C. Lyzell: Initialization Methods for System Identification. Thesis No. 1426, 2009.

R. Falkeborn: Structure exploitation in semidefinite programming for control. Thesis No. 1430, 2010.

D. Petersson: Nonlinear Optimization Approaches to $\mathcal{H}_{2}$-Norm Based LPV Modelling and Control. Thesis No. 1453, 2010.

Z. Sjanic: Navigation and SAR Auto-focusing in a Sensor Fusion Framework. Thesis No. 1464, 2011.

K. Granström: Loop detection and extended target tracking using laser data. Thesis No. 1465, 2011.

J. Callmer: Topics in Localization and Mapping. Thesis No. 1489, 2011.

F. Lindsten: Rao-Blackwellised particle methods for inference and identification. Thesis No. 1480, 2011.

M. Skoglund: Visual Inertial Navigation and Calibration. Thesis No. 1500, 2011.

S. Khoshfetrat Pakazad: Topics in Robustness Analysis. Thesis No. 1512, 2011.

P. Axelsson: On Sensor Fusion Applied to Industrial Manipulators. Thesis No. 1511, 2011.

A. Carvalho Bittencourt: On Modeling and Diagnosis of Friction and Wear in Industrial Robots. Thesis No. 1516, 2012.

P. Rosander: Averaging level control in the presence of frequent inlet flow upsets. Thesis No. 1527, 2012. 
N. Wahlström: Localization using Magnetometers and Light Sensors. Thesis No. 1581, 2013.

R. Larsson: System Identification of Flight Mechanical Characteristics. Thesis No. 1599, 2013.

Y. Jung: Estimation of Inverse Models Applied to Power Amplifier Predistortion. Thesis No. 1605, 2013.

M. Syldatk: On Calibration of Ground Sensor Networks. Thesis No. 1611, 2013.

M. Roth: Kalman Filters for Nonlinear Systems and Heavy-Tailed Noise. Thesis No. 1613, 2013.

D. Simon: Model Predictive Control in Flight Control Design - Stability and Reference Tracking. Thesis No. 1642, 2014.

J. Dahlin: Sequential Monte Carlo for inference in nonlinear state space models. Thesis No. 1652, 2014.

M. Kok: Probabilistic modeling for positioning applications using inertial sensors. Thesis No. 1656, 2014.

J. Linder: Graybox Modelling of Ships Using Indirect Input Measurements. Thesis No. 1681, 2014.

G. Mathai: Direction of Arrival Estimation of Wideband Acoustic Wavefields in a Passive Sensing Environment. Thesis No. 1721, 2015.

I. Nielsen: On Structure Exploiting Numerical Algorithms for Model Predictive Control. Thesis No. 1727, 2015.

C. Veibäck: Tracking of Animals Using Airborne Cameras. Thesis No. 1761, 2016.

N. Evestedt: Sampling Based Motion Planning for Heavy Duty Autonomous Vehicles. Thesis No. 1762, 2016.

H. Nyqvist: On Pose Estimation in Room-Scaled Environments. Thesis No. 1765, 2016.

Y. Zhao: Position Estimation in Uncertain Radio Environments and Trajectory Learning. Thesis No. 1772, 2017.

P. Kasebzadeh: Parameter Estimation for Mobile Positioning Applications. Thesis No. 1786, 2017.

K. Radnosrati: On Timing-Based Localization in Cellular Radio Networks. Thesis No. 1808, 2018.

G. Lindmark: Methods and Algorithms for Control Input Placement in Complex Networks. Thesis No. 1814, 2018.

M. Lindfors: Frequency Tracking for Speed Estimation. Thesis No. 1815, 2018.

D. Ho: Some results on closed-loop identification of quadcopters. Thesis No. 1826, 2018.

O. Ljungqvist: On motion planning and control for truck and trailer systems. Thesis No. 1832, 2019.

P. Boström-Rost: On Informative Path Planning for Tracking and Surveillance. Thesis No. 1838, 2019.

K. Bergman: On Motion Planning Using Numerical Optimal Control. Thesis No. 1843, 2019.

M. Klingspor: Low-rank optimization in system identification. Thesis No. 1855, 2019.

A. Bergström: Timing-Based Localization using Multipath Information. Thesis No. 1867, 2019.

F. Ljungberg: Estimation of Nonlinear Greybox Models for Marine Applications. Thesis No. 1880, 2020.

E. Hedberg: Control, Models and Industrial Manipulators. Thesis No. 1894, 2020.

R. Forsling: Decentralized Estimation Using Conservative Information Extraction. Thesis No. 1897, 2020. 
D. Arnström: On Complexity Certification of Active-Set QP Methods with Applications to Linear MPC. Thesis No. 1901, 2021.

M. Malmström: Uncertainties in Neural Networks: A System Identification Approach. Thesis No. 1902, 2021.

K. Nielsen: Robust LIDAR-Based Localization in Underground Mines. Thesis No. 1906, 2021.

H. Haghshenas: Time-Optimal Cooperative Path Tracking for Multi-Robot Systems. Thesis No. 1915, 2021.

A. Kullberg: On Joint State Estimation and Model Learning using Gaussian Process Approximations. Thesis No. 1917, 2021. 


\section{FACULTY OF SCIENCE AND ENGINEERING}

Linköping studies in science and technology, Licentiate Thesis No. 1927 Department of Electrical Engineering

Linköping University

SE-581 83 Linköping, Sweden

www.liu.se 